Check for updates

Cite this: New J. Chem., 2022, 46,6899

Received 19th January 2022, Accepted 1st March 2022

DOI: 10.1039/d2nj00296e

rsc.li/njc

\section{Structural diversity and magnetic properties of copper(II) quinaldinate compounds with amino alcohols $\dagger$}

\author{
Nina Podjed, (DD ${ }^{a}$ Barbara Modec, (D)*a Rodolphe Clérac, (D) ${ }^{b}$ Mathieu Rouzières, ${ }^{b}$ \\ María M. Alcaide ${ }^{c}$ and Joaquín López-Serrano (D) ${ }^{c}$
}

\begin{abstract}
The reactions between $\left[\mathrm{Cu}(\text { quin) })_{2}\left(\mathrm{H}_{2} \mathrm{O}\right)\right]$ (quin ${ }^{-}=$the anionic form of quinoline-2-carboxylic acid) and a series of aliphatic amino alcohols have yielded structurally very diverse copper(॥) complexes, labelled a-g. Single-crystal $X$-ray structure analysis has revealed either intact amino alcohol molecules or amino alcoholate ions serving as ligands. In type a complexes, the amino alcohols are bound in a monodentate manner via $\mathrm{NH}_{2}$. Engagement of both functional groups in coordination was observed for types $\mathbf{b}$ and $\mathbf{e}$ (a bidentate chelating mode) and type c (a bidentate bridging one) complexes. In view of the strong bidentate chelating coordination of quinaldinate in $\left[\mathrm{Cu}(\text { quin })_{2}\left(\mathrm{H}_{2} \mathrm{O}\right)\right]$, the formation of homoleptic amino alcohol complexes e was not anticipated. Equally surprising was the transformation of a mononuclear starting material into a one-dimensional (1D) coordination polymer, [Cu(quin) $]_{n}$ (g). Spontaneous deprotonation of some amino alcohols and coordination of, thus formed, amino alcoholates via both donors also took place. Dinuclear complexes (d) contained two bridging amino alcoholates, whilst bidentate chelating mode was observed for type f. Interestingly, the dinuclear complex exists as two isomers which differ in the position of quinaldinates with respect to the $\mathrm{Cu}(\mu-\mathrm{OR})_{2} \mathrm{Cu}$ core. DFT

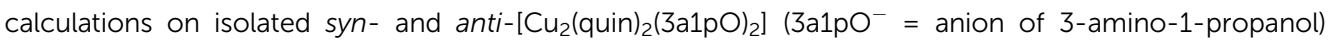
have shown the syn isomer to be more stable. The explanation lies in the intramolecular $\pi \cdots \pi$ stacking of quinaldinates, possible only in this isomer. Magnetic susceptibility measurements revealed antiferromagnetic interactions between $S=1 / 2$ copper(II) spins in all the studied compounds except in $\left[\mathrm{Cu}(\text { quin })_{2}\right]_{n}$ (g) for which weak ferromagnetic couplings are detected.
\end{abstract}

\section{Introduction}

The last two decades have witnessed remarkable progress in crystal engineering. ${ }^{1-3}$ As follows from its definition, the design of molecular solids with specific physical and chemical

${ }^{a}$ Faculty of Chemistry and Chemical Technology, University of Ljubljana, Večna pot 113, 1000 Ljubljana, Slovenia. E-mail: barbara.modec@fkkt.uni-lj.si

${ }^{b}$ Univ. Bordeaux, CNRS, Centre de Recherche Paul Pascal, UMR 5031, F-33600, Pessac, France

${ }^{c}$ Instituto de Investigaciones Quimicas (IIQ), Departamento de Quimica Inorgánica and Centro de Innovación en Quimica Avanzada (ORFEO-CINQA), Consejo Superior de Investigaciones Científicas (CSIC) and Universidad de Sevilla, Avenida Américo Vespucio 49, 41092 Sevilla, Spain

$\dagger$ Electronic supplementary information (ESI) available: Powder X-ray diffraction patterns (Fig. S1), crystallographic data (Tables S1-S3), ORTEP drawings of 1a and syn-8d (Fig. S2 and S3), intermolecular interactions (Fig. S4-S19 and Tables S5-S13), additional geometric parameters (Tables S4 and S14), details on DFT calculations (Tables S15-S17), optimized geometries and infrared spectroscopy data (Tables S18-S19, Fig. S20-S44). CCDC 2076993-2077015. For ESI and crystallographic data in CIF or other electronic format see DOI: 10.1039/d2nj00296e properties through understanding and control of intermolecular interactions, ${ }^{4,5}$ crystal engineering encompasses many aspects of classical coordination chemistry. The choice of the metal ion is crucial as the metal performs a functional role of the solid material through its redox, magnetic and optical properties. Copper(II) is one of the frequently employed metal ions in crystal engineering because of its versatile coordination behavior, redox and catalytic properties. It has a $\mathrm{d}^{9}$ electron configuration with geometric preferences based partly on LFSE (ligand field stabilization energy): it is normally coordinated by 4,5 or 6 donors in square-planar, distorted square-pyramidal or, owing to Jahn-Teller distortion, axially distorted octahedral geometries. ${ }^{6}$ All the above properties combined with lower toxicity than most $4 \mathrm{~d}$ and $5 \mathrm{~d}$ transition metals ${ }^{7}$ make copper(II) a widespread ion in nature. It is present in active sites of many oxidation enzymes. ${ }^{8}$ The involvement of copper ions in electron and oxygen transport exploits the easy interconversion between the +1 and +2 oxidation states. $^{9}$

Amino alcohols are a class of multidentate $\mathrm{N}, \mathrm{O}$-donor ligands that have also been used in crystal engineering. Both their 
functional groups, $\mathrm{OH}$ and $\mathrm{NH}_{2}$, are able to coordinate to transition metal ions. The $\mathrm{OH}$ moiety can give off its proton, producing anions which act as bridging and chelating ligands. Studies of copper(II) coordination chemistry with amino alcohols have shown that the $\mathrm{OH}$ group frequently undergoes spontaneous 0deprotonation. ${ }^{10}$ Another important characteristic of amino alcohols that makes them suitable for application in crystal engineering is their ability to participate in hydrogen bonding. The above listed properties combined with their commercial availability and low cost have strongly encouraged research on their coordination chemistry with transition metal ions including copper(II). ${ }^{11}$ Studies with a number of aliphatic amino alcohols have been performed so far, e.g., 2-aminoethanol, ${ }^{12} N, N^{\prime}$-dimethylethanolamine, ${ }^{13}$ 3-amino-1-propanol, ${ }^{10,12,14-20}$ diethanolamine,${ }^{21-23} \mathrm{~N}$-methyldiethanolamine, ${ }^{24-26} \mathrm{~N}$-butyldiethanolamine, ${ }^{13}$ and triethanolamine. ${ }^{27,28}$ A survey of the Cambridge Structural Database reveals that there are more complexes with amino alcoholate ions than with intact amino alcohols. ${ }^{29} \mathrm{~A}$ recent review describes the structural diversity of copper(II) amino alcoholate complexes. ${ }^{30}$ The most common structural type encountered is $\mathrm{Cu}(\mu-\mathrm{OR})_{2} \mathrm{Cu}$, a dinuclear core with copper(II) ions bridged by two amino alcoholate $\left({ }^{-} \mathrm{OR}\right)$ oxygen atoms. Owing to their rigid behavior, the dinuclear cores served as building units in the construction of metal-organic frameworks (MOFs) ${ }^{31,32}$ whose dimensionality was controlled through the denticity of the parent amino alcohol. ${ }^{23}$ It is noteworthy that some copper(II) amino alcoholate complexes have found application in catalysis, ${ }^{33}$ as exemplified by their catalytic role in the oxidative transformation of alkanes. ${ }^{34-36}$

We reported recently on the reactions of zinc(II) quinaldinate with two amino alcohols, 3-amino-1-propanol and 2-methylaminoethanol. ${ }^{37}$ Through the use of quinaldinate (abbreviated throughout the text as quin ${ }^{-}$), the anionic form of quinaldinic acid (Scheme 1), which usually binds in a $\mathrm{N}, \mathrm{O}$-chelating manner and thereby occupies two binding sites in the metal's coordination sphere, the possible reaction outcomes were highly limited. As a follow-up, zinc(II) was replaced with copper(II) which is known for its redox activity. Another reason for choosing copper(II) was that in spite of its radically different chemical behavior, copper(II) forms many homologous compounds to zinc(II). The study was expanded also by using a larger assortment of amino alcohols. Despite the recognized use of many amino alcohols, their combination with quinaldinate in copper(II) coordination chemistry has not been explored and no copper(II) compounds containing both ligands have been documented. The selected amino alcohols include primary amines (3-amino-1-propanol, 2-aminoethanol, 1-amino-2-propanol, 1-amino-2-methyl-2-propanol, 1-amino-2-butanol, 2-amino-1propanol, 2-amino-2-methyl-1-propanol, 2-amino-1-butanol),

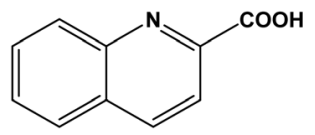

Scheme 1 Structural formula of quinoline-2-carboxylic acid, also known as quinaldinic acid. secondary amines (2-methylaminoethanol and 2-ethylaminoethanol) and a tertiary amine (2-dimethylaminoethanol). The latter three amino alcohols were chosen in order to explore the influence of an alkyl substituent on nitrogen atoms over their coordination behavior. The structural formulae of the used amino alcohols, their names and the abbreviations are given in Scheme 2. Herein, we report on the products of threecomponent copper(II)-quinaldinate-amino alcohol systems. Their detailed characterization by single-crystal X-ray diffraction analyses and infrared vibrational spectroscopy is presented. For convenience, the novel compounds are classified into seven structural types, labelled a to $\mathbf{g}$ (Scheme 3 ). Type a denotes trans-[Cu(quin) $\left.)_{2} \mathrm{~L}_{2}\right](\mathrm{L}=$ amino alcohol) complexes. Although type $\mathbf{b}$ and $\mathbf{c}$ share the same composition, [Cu(quin) $\left.{ }_{2} \mathrm{~L}\right]$, they differ in the amino alcohol binding mode and therefore in dimensionality. In a mononuclear type $\mathbf{b}$ complex, the amino alcohol adopts a bidentate chelating coordination mode, whereas in $\mathbf{c}$ with an infinite chain structure the amino alcohol serves as a bidentate bridging ligand. A type d dinuclear complex with bridging amino alcoholates exists in two isomeric forms, syn and anti, which differ in the quinaldinate position with respect to the $\mathrm{Cu}(\mu-\mathrm{O})_{2} \mathrm{Cu}$ plane. Type e compounds with the $\left[\mathrm{CuL}_{3}\right]^{2+}$ formula are homoleptic amino alcohol complexes, whereas type $\mathbf{f}$ is a homoleptic amino alcoholate complex with a metal ion in a square-planar environment. Finally, type $\mathbf{g}$ is a homoleptic quinaldinate complex with an infinite chain structure. With the exception of type compounds, the magnetic properties were studied on at least one compound of each type. A correlation between their magnetic behavior and solid-state structures is presented. In addition, DFT calculations were

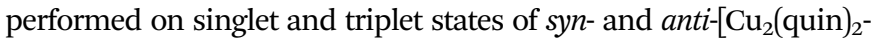
$\left.(3 \mathrm{a} 1 \mathrm{pO})_{2}\right]$. The calculations on selected $3 \mathrm{a} 1 \mathrm{pOH}$ complexes were used to assess their stabilities.

\section{Experimental}

\section{General}

Acetonitrile, used as a solvent, was dried with molecular sieves. ${ }^{38}$ Other reagents were purchased from commercial sources and used as received. The synthetic procedure for the starting material $\left[\mathrm{Cu}(\text { quin })_{2}\left(\mathrm{H}_{2} \mathrm{O}\right)\right]$ was the same as described previously. ${ }^{39}$ A Bruker Alpha II FT-IR spectrophotometer with an ATR module was used to record infrared spectra in the 4000$400 \mathrm{~cm}^{-1}$ spectral range. No manipulations were made on the IR spectra. Powder X-ray diffraction (PXRD) patterns for aged anti-11d-4MeOH were obtained using a PANanlytical X'Pert PRO MD diffractometer using monochromatised $\mathrm{Cu}-\mathrm{K}_{\alpha}$ radiation $(\lambda=1.5406 \AA)$. A PerkinElmer 2400 II analyzer was used for elemental $\mathrm{CHN}$ analyses. Elemental analyses were not performed for 11a.3a1pOH, 11b, syn-11d, anti-11d.4MeOH, anti11d-2MeCN, and anti-11d-2MeOH·2(3a1pOH) for three different reasons. 11a.3a1pOH, anti-11d.4MeOH, anti-11d.2MeCN, and anti-11d·2MeOH.2(3a1pOH) are solvates which are not stable outside of the mother liquor, 11b was obtained as a one-time event in a very small amount, whereas $\boldsymbol{s y n - 1 1 d}$ was 

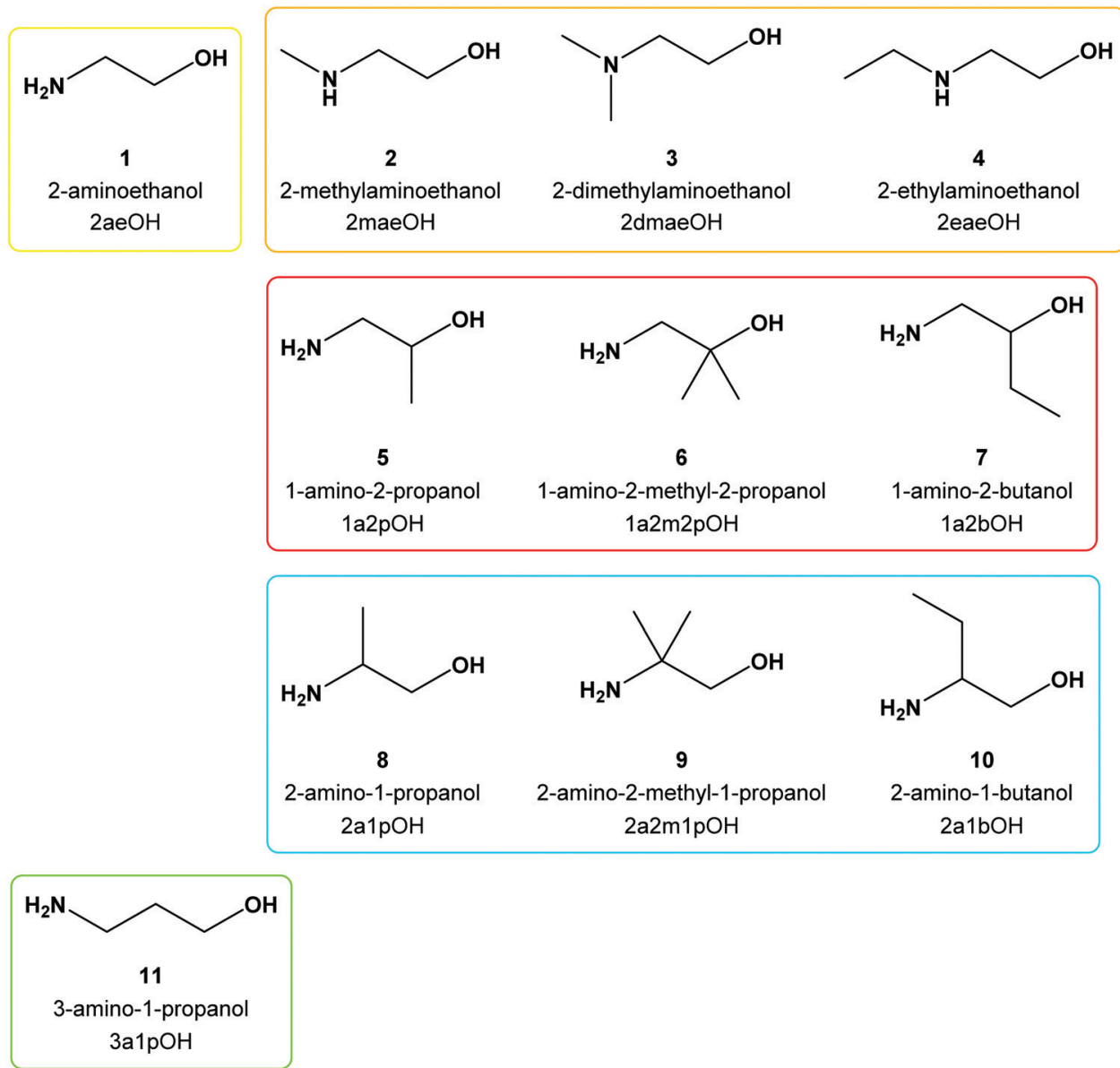

Scheme 2 Amino alcohols used in this study.

obtained in a mixture with other compounds. All novel compounds except $\boldsymbol{s y n}$-9d and $\boldsymbol{s y n}$-10d were characterized by X-ray structure analysis on single-crystals.

\section{trans-[Cu(quin $\left.)_{2}(\mathbf{2 a e O H})_{2}\right](\mathbf{1 a})$}

Procedure A. A Teflon container was filled with $\left[\mathrm{Cu}(\text { quin })_{2}\left(\mathrm{H}_{2} \mathrm{O}\right)\right]$ $(50 \mathrm{mg}, 0.12 \mathrm{mmol})$, acetonitrile $(7.5 \mathrm{~mL})$ and 2 -aminoethanol $(1 \mathrm{~mL})$. The container was closed and inserted into a steel autoclave, which was heated for 24 hours at $105{ }^{\circ} \mathrm{C}$. Afterwards, the autoclave was taken out from the oven and left overnight to cool to room temperature. Green, needle-like crystals of trans$\left.[\mathrm{Cu} \text { (quin })_{2}(2 \mathrm{aeOH})_{2}\right](1 \mathrm{a})$ were filtered off. Yield: $41 \mathrm{mg}, 66 \%$. Procedure $B$. $\left[\mathrm{Cu}(\text { quin })_{2}\left(\mathrm{H}_{2} \mathrm{O}\right)\right](50 \mathrm{mg}, 0.12 \mathrm{mmol})$, acetonitrile $(7.5 \mathrm{~mL})$ and 2-aminoethanol $(1 \mathrm{~mL})$ were added to an Erlenmeyer flask. The flask was closed and left to stand under ambient conditions. On the following day, blue crystalline precipitate was filtered off and air-dried. Yield: $53 \mathrm{mg}, 85 \%$. Please note that the same product formed at $4{ }^{\circ} \mathrm{C}$. IR (ATR, $\mathrm{cm}^{-1}$ ): 3284s, 3156w, 3110w, 3087w, 3063w, 2971w, 2958m, $2859 \mathrm{w}, 2715 \mathrm{w}, 1604 \mathrm{vs}, 1587 \mathrm{vs}, 1562 \mathrm{~s}, 1550 \mathrm{~s}, 1505 \mathrm{~m}, 1461 \mathrm{~s}$, $1430 \mathrm{~m}, 1372 \mathrm{vs}, 1345 \mathrm{~s}, 1301 \mathrm{w}, 1268 \mathrm{w}, 1254 \mathrm{~m}, 1218 \mathrm{~m}, 1206 \mathrm{w}$, $1174 \mathrm{~s}, 1152 \mathrm{~m}, 1142 \mathrm{w}, 1117 \mathrm{w}, 1079 \mathrm{~s}, 1034 \mathrm{vvs}, 1021 \mathrm{~s}, 994 \mathrm{w}$, $957 \mathrm{~m}, 896 \mathrm{~m}, 881 \mathrm{~m}, 859 \mathrm{~s}, 854 \mathrm{~s}, 810 \mathrm{~s}, 798 \mathrm{~s}, 777 \mathrm{vvs}, 748 \mathrm{~s}$, 708s, 632s, 607vs, 601vvs, 576s, 548m, 522m, 494s, 482m, 443w, 413m. Elemental analysis calcd. for $\mathrm{C}_{24} \mathrm{H}_{26} \mathrm{CuN}_{4} \mathrm{O}_{6}$ (\%): C, 54.39; H, 4.94; N, 10.57. Found (\%): C, 54.03; H, 4.73; $\mathrm{N}, 10.72$.

\section{[Cu(quin) $\left.)_{2}(2 \mathrm{maeOH})\right](2 \mathrm{~b})$}

Procedure A. A Teflon container was filled with $\left[\mathrm{Cu}(\text { quin })_{2}\left(\mathrm{H}_{2} \mathrm{O}\right)\right]$ $(50 \mathrm{mg}, 0.12 \mathrm{mmol})$, acetonitrile $(7.5 \mathrm{~mL})$ and 2-methylaminoethanol $(0.25 \mathrm{~mL})$. The container was closed and inserted into a steel autoclave, which was heated for 24 hours at $105^{\circ} \mathrm{C}$. Afterwards, the autoclave was taken out from the oven and left overnight to cool to room temperature. Green crystals of $\left.[\mathrm{Cu} \text { (quin })_{2}(2 \mathrm{maeOH})\right](2 \mathbf{b})$ were filtered off. Yield: $50 \mathrm{mg}, 88 \%$. Procedure $B .\left[\mathrm{Cu}(\text { quin })_{2}\left(\mathrm{H}_{2} \mathrm{O}\right)\right](50 \mathrm{mg}, 0.12 \mathrm{mmol})$, acetonitrile $(7.5 \mathrm{~mL})$ and 2-methylaminoethanol $(0.25 \mathrm{~mL})$ were added to an Erlenmeyer flask. The flask was closed and left to stand under ambient conditions. On the following day, blue crystalline solid was filtered off and air-dried. Yield: $51 \mathrm{mg}, 90 \%$. Please note that the same product formed at $4{ }^{\circ} \mathrm{C}$. IR (ATR, $\mathrm{cm}^{-1}$ ): 3378w, 3211m, 3103w, 3054w, 3007w, 2970w, 2904w, $2850 \mathrm{w}, 1640 \mathrm{~s}, 1594 \mathrm{~m}, 1564 \mathrm{~m}, 1509 \mathrm{w}, 1484 \mathrm{w}, 1461 \mathrm{~m}, 1415 \mathrm{w}$, $1362 \mathrm{vs}, 1343 \mathrm{~s}, 1295 \mathrm{~m}, 1268 \mathrm{~m}, 1216 \mathrm{~m}, 1183 \mathrm{~m}, 1172 \mathrm{~m}, 1150 \mathrm{~m}$, $1112 \mathrm{w}, 1094 \mathrm{w}, 1058 \mathrm{~m}, 1029 \mathrm{~s}, 979 \mathrm{w}, 969 \mathrm{w}, 958 \mathrm{w}, 899 \mathrm{~s}, 877 \mathrm{~m}$, $850 \mathrm{~m}, 800 \mathrm{vs}, 780 \mathrm{vvs}, 662 \mathrm{~m}, 640 \mathrm{~m}, 628 \mathrm{~s}, 606 \mathrm{~s}, 563 \mathrm{w}, 550 \mathrm{~m}$, $522 \mathrm{~m}, 497 \mathrm{~s}, 481 \mathrm{~m}, 413 \mathrm{~m}$. Elemental analysis calcd for 


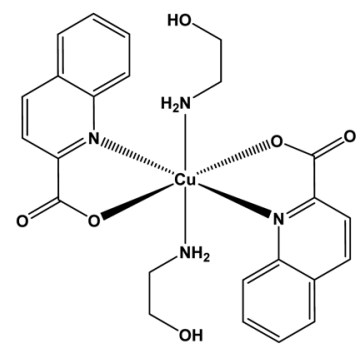

type a $L=2 a e O H$

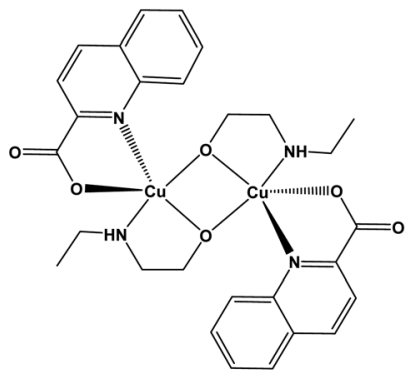

$$
\text { type d }
$$
$\mathrm{L}=2 \mathrm{eae} \mathrm{O}^{-}$<smiles>CC1C[C@H](O)C(OC(=O)c2ccc3ccccc3n2)(OC(=O)c2ccc3ccccc3n2)N1</smiles>

type $\mathbf{b}$ $\mathrm{L}=2 \mathrm{a} 1 \mathrm{pOH}$

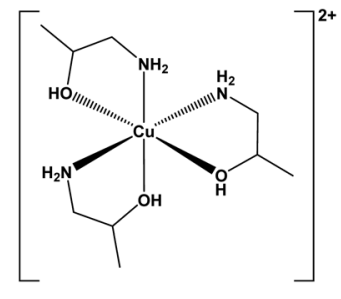

type $\mathbf{e}$ $L=1 \mathrm{a} 2 \mathrm{pOH}$

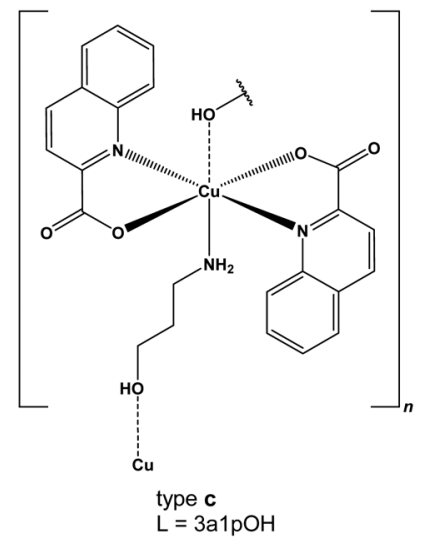

type $\mathbf{f}$ $L=2 \mathrm{a} 2 \mathrm{~m} 1 \mathrm{pO}-$

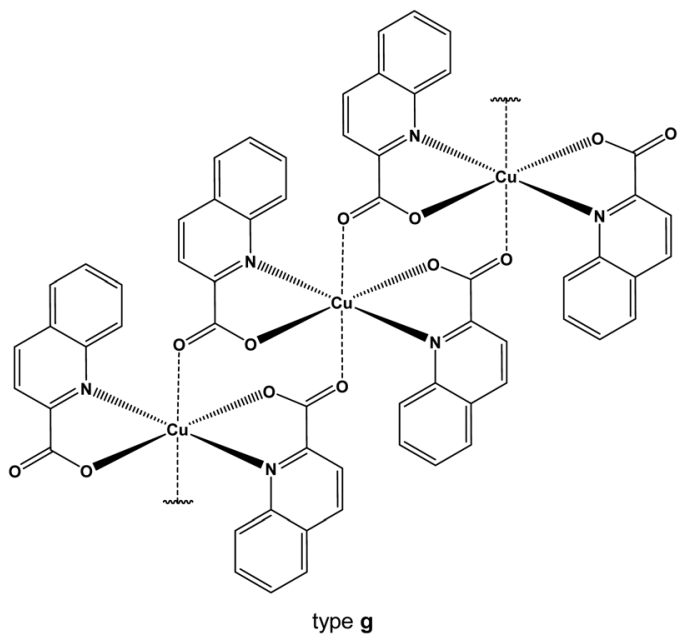

Scheme 3 Structural formulae of novel complexes. L denotes the amino alcohol ligand.

$\mathrm{C}_{23} \mathrm{H}_{21} \mathrm{CuN}_{3} \mathrm{O}_{5}$ (\%): C, 57.20; H, 4.38; N, 8.70. Found (\%): C, 57.12; H, 4.21; N, 8.76.

\section{$\left[\mathrm{Cu}(\text { quin })_{2}\right]_{n}(\mathrm{~g})$}

A Teflon container was filled with $\left[\mathrm{Cu}(\text { quin })_{2}\left(\mathrm{H}_{2} \mathrm{O}\right)\right](50 \mathrm{mg}$, $0.12 \mathrm{mmol})$, acetonitrile $(7.5 \mathrm{~mL})$ and 2-dimethylaminoethanol $(0.1 \mathrm{~mL})$. The container was closed and inserted into a steel autoclave, which was heated for 24 hours at $105^{\circ} \mathrm{C}$. Afterwards, the autoclave was taken out from the oven and left overnight to cool to room temperature. Green crystals of $\left[\mathrm{Cu}(\text { quin })_{2}\right]_{n}$ (g) were filtered off and air-dried. Yield: $39 \mathrm{mg}, 81 \%$. Please note that the reaction without the amino alcohol yielded $\left.[\mathrm{Cu} \text { (quin) })_{2}\right]_{n}$ (g) with approximately the same yield. On prolonged standing in the solution, compound $\mathbf{g}$ converted into a starting material, $\left[\mathrm{Cu}(\text { quin })_{2}\left(\mathrm{H}_{2} \mathrm{O}\right)\right]$. IR (ATR, $\left.\mathrm{cm}^{-1}\right): 3132 \mathrm{w}, 3102 \mathrm{w}, 3080 \mathrm{w}, 3051 \mathrm{w}$, $3015 \mathrm{w}, 1637 \mathrm{~s}, 1596 \mathrm{~m}, 1567 \mathrm{~m}, 1556 \mathrm{~m}, 1510 \mathrm{~m}, 1463 \mathrm{~m}, 1436 \mathrm{w}$, $1383 \mathrm{~s}, 1372 \mathrm{~s}, 1357 \mathrm{~s}, 1274 \mathrm{~m}, 1211 \mathrm{~m}, 1202 \mathrm{~m}, 1187 \mathrm{~m}, 1148 \mathrm{~m}$, $1112 \mathrm{w}, 1026 \mathrm{~m}, 1000 \mathrm{w}, 966 \mathrm{~m}, 904 \mathrm{~m}, 881 \mathrm{~m}, 853 \mathrm{~s}, 805 \mathrm{vs}, 767 \mathrm{vvs}$, 741s, 735s, 641s, 633s, 611s, 576m, 522m, 493vs, 479m, 436s, 405s. Elemental analysis calcd for $\mathrm{C}_{20} \mathrm{H}_{12} \mathrm{CuN}_{2} \mathrm{O}_{4}$ (\%): C, 58.90; H, 2.97; N, 6.87. Found (\%): C, 58.82; H, 2.93; N, 6.77.

\section{anti-[Cu (quin $\left._{2}(2 \mathrm{eaeO})_{2}\right]($ anti-4d)}

Procedure A. A Teflon container was filled with $\left[\mathrm{Cu}(\text { quin })_{2}\left(\mathrm{H}_{2} \mathrm{O}\right)\right]$ (50 $\mathrm{mg}, 0.12 \mathrm{mmol})$, acetonitrile $(7.5 \mathrm{~mL})$ and 2-ethylaminoethanol $(1 \mathrm{~mL})$. The container was closed and inserted into a 
steel autoclave, which was heated for 24 hours at $105{ }^{\circ} \mathrm{C}$. Afterwards, the autoclave was taken out from the oven and left overnight to cool to room temperature. Green needle-like

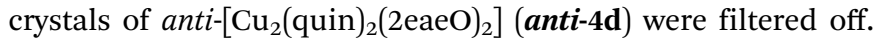
Yield: $14 \mathrm{mg}, 37 \%$. Procedure $B$. [Cu(quin $\left.)_{2}\left(\mathrm{H}_{2} \mathrm{O}\right)\right](50 \mathrm{mg}$, $0.12 \mathrm{mmol})$, acetonitrile $(7.5 \mathrm{~mL})$ and 2-ethylaminoethanol $(0.5 \mathrm{~mL})$ were added to an Erlenmeyer flask. The flask was closed and left to stand under ambient conditions. On the following day, green crystalline solid was filtered off and airdried. Yield: $34 \mathrm{mg}, 89 \%$. Please note that the same product formed at $4{ }^{\circ} \mathrm{C}$. IR (ATR, $\mathrm{cm}^{-1}$ ): $3183 \mathrm{~m}, 3061 \mathrm{w}, 2975 \mathrm{w}, 2938 \mathrm{~m}$, $2876 \mathrm{~m}, 2833 \mathrm{~m}, 1636 \mathrm{vvs}, 1595 \mathrm{~m}, 1566 \mathrm{~m}, 1507 \mathrm{w}, 1464 \mathrm{~m}, 1432 \mathrm{w}$, $1360 \mathrm{vs}, 1306 \mathrm{w}, 1262 \mathrm{w}, 1219 \mathrm{~m}, 1173 \mathrm{~s}, 1153 \mathrm{~m}, 1137 \mathrm{w}, 1094 \mathrm{~m}$, 1062s, 1032s, 993w, 974w, 961w, 929w, 912m, 897m, 876m, $848 \mathrm{~s}, 800 \mathrm{~s}, 781 \mathrm{vs}, 775 \mathrm{vs}, 738 \mathrm{w}, 629 \mathrm{~s}, 600 \mathrm{~s}, 553 \mathrm{~m}, 522 \mathrm{w}, 490 \mathrm{~m}$, $450 \mathrm{~s}, 412 \mathrm{~s}$. Elemental analysis calcd for $\mathrm{C}_{28} \mathrm{H}_{32} \mathrm{Cu}_{2} \mathrm{~N}_{4} \mathrm{O}_{6}(\%)$ : C, 51.93; H, 4.98; N, 8.65. Found (\%): C, 51.98; H, 4.88; N, 8.81.

\section{$\left[\mathrm{Cu}(\mathbf{1 a 2 p O H})_{3}\right](\text { quin })_{2}(5 \mathrm{e})$}

Procedure A. A Teflon container was filled with $\left[\mathrm{Cu}(\text { quin })_{2}\left(\mathrm{H}_{2} \mathrm{O}\right)\right]$ (50 $\mathrm{mg}, 0.12 \mathrm{mmol})$, acetonitrile $(7.5 \mathrm{~mL})$ and 1-amino-2propanol $(0.5 \mathrm{~mL})$. The container was closed and inserted into a steel autoclave, which was heated for 24 hours at $105{ }^{\circ} \mathrm{C}$. Afterwards, the autoclave was taken out from the oven and left overnight to cool to room temperature. Blue needle-like crystals of $\left[\mathrm{Cu}(1 \mathrm{a} 2 \mathrm{pOH})_{3}\right](\text { quin })_{2}$ (5e) were filtered off. Yield: $60 \mathrm{mg}$, $81 \%$. Procedure B. [Cu(quin $\left.)_{2}\left(\mathrm{H}_{2} \mathrm{O}\right)\right](50 \mathrm{mg}, 0.12 \mathrm{mmol})$, acetonitrile $(7.5 \mathrm{~mL})$ and 1-amino-2-propanol $(0.5 \mathrm{~mL})$ were added to an Erlenmeyer flask. The flask was closed and left to stand under ambient conditions. On the following day, blue crystalline solid was filtered off and air-dried. Yield: $72 \mathrm{mg}, 97 \%$. Please note that the same product formed at $4{ }^{\circ} \mathrm{C}$. IR (ATR, $\mathrm{cm}^{-1}$ ): 3236m, 3122m, 2971w, 2963w, 2927w, 2873w, 1829w, $1615 \mathrm{~s}, 1595 \mathrm{~m}, 1581 \mathrm{~s}, 1553 \mathrm{~s}, 1500 \mathrm{~m}, 1462 \mathrm{~m}, 1424 \mathrm{~m}, 1380 \mathrm{~s}$, 1369vs, 1341s, 1300m, 1213w, 1199w, 1171s, 1145w, 1117s, $1108 \mathrm{~s}, 1090 \mathrm{~s}, 1079 \mathrm{~s}, 1058 \mathrm{~m}, 1026 \mathrm{~s}, 993 \mathrm{w}, 956 \mathrm{w}, 925 \mathrm{w}, 912 \mathrm{w}$, $890 \mathrm{~m}, 876 \mathrm{w}, 856 \mathrm{~m}, 832 \mathrm{w}, 809 \mathrm{~s}, 777 \mathrm{vvs}, 739 \mathrm{~s}, 707 \mathrm{~m}, 644 \mathrm{~m}$, $627 \mathrm{~m}, 595 \mathrm{~s}, 565 \mathrm{w}, 548 \mathrm{w}, 535 \mathrm{w}, 520 \mathrm{~m}, 500 \mathrm{w}, 485 \mathrm{~m}, 476 \mathrm{~m}, 408 \mathrm{w}$. Elemental analysis calcd for $\mathrm{C}_{29} \mathrm{H}_{39} \mathrm{CuN}_{5} \mathrm{O}_{7}$ (\%): C, 55.01; H, 6.21; N, 11.06. Found (\%): C, 54.96; H, 6.11; N, 11.11 .

\section{trans $-\left[\mathrm{Cu}(\text { quin })_{2}(1 \mathrm{a} 2 \mathrm{~m} 2 \mathrm{pOH})_{2}\right](6 \mathrm{a})$}

Procedure A. A Teflon container was filled with $\left[\mathrm{Cu}(\text { quin })_{2}\left(\mathrm{H}_{2} \mathrm{O}\right)\right]$ (50 $\mathrm{mg}, 0.12 \mathrm{mmol})$, acetonitrile $(7.5 \mathrm{~mL})$ and 1-amino-2methyl-2-propanol $(0.25 \mathrm{~mL})$. The container was closed and inserted into a steel autoclave, which was heated for 24 hours at $105{ }^{\circ} \mathrm{C}$. Afterwards, the autoclave was taken out from the oven and left overnight to cool to room temperature. Blue needle-like crystals of trans $-\left[\mathrm{Cu}(\text { quin })_{2}(1 \mathrm{a} 2 \mathrm{~m} 2 \mathrm{pOH})_{2}\right](\mathbf{6 a})$ were filtered off. Yield: $62 \mathrm{mg}$, 90\%. Procedure B. [Cu(quin $\left.)_{2}\left(\mathrm{H}_{2} \mathrm{O}\right)\right](50 \mathrm{mg}$, $0.12 \mathrm{mmol})$, acetonitrile $(7.5 \mathrm{~mL})$ and 1-amino-2-methyl-2propanol $(0.5 \mathrm{~mL})$ were added to an Erlenmeyer flask. The flask was closed and left to stand under ambient conditions. On the following day, blue crystalline solid was filtered off and air-dried. Yield: $66 \mathrm{mg}, 96 \%$. Please note that the same product formed at $4{ }^{\circ} \mathrm{C}$. IR (ATR, $\mathrm{cm}^{-1}$ ): 3323m, 3244w, 3148w, 3060w,
$2974 \mathrm{~m}, 2964 \mathrm{w}, 2933 \mathrm{w}, 1609 \mathrm{~s}, 1587 \mathrm{~s}, 1561 \mathrm{~s}, 1548 \mathrm{~m}, 1505 \mathrm{~m}$, $1461 \mathrm{~m}, 1430 \mathrm{w}, 1404 \mathrm{~m}, 1379 \mathrm{~s}, 1369 \mathrm{~s}, 1336 \mathrm{~s}, 1265 \mathrm{~m}, 1250 \mathrm{~m}$, $1217 \mathrm{~m}, 1178 \mathrm{~s}, 1142 \mathrm{~s}, 1105 \mathrm{~s}, 1037 \mathrm{~s}, 1018 \mathrm{~m}, 997 \mathrm{~m}, 971 \mathrm{~m}, 958 \mathrm{~m}$, $932 \mathrm{w}, 907 \mathrm{~m}, 896 \mathrm{~s}, 878 \mathrm{~m}, 869 \mathrm{~m}, 859 \mathrm{~m}, 808 \mathrm{~s}, 798 \mathrm{~s}, 774 \mathrm{vvs}, 745 \mathrm{~s}$, 672m, 632vs, 601vs, 589s, 547m, 522m, 495m, 481m, 415w. Elemental analysis calcd for $\mathrm{C}_{28} \mathrm{H}_{34} \mathrm{CuN}_{4} \mathrm{O}_{6}$ (\%): C, 57.38; H, 5.85; N, 9.56. Found (\%): C, 57.18; H, 5.78; N, 9.54.

\section{trans-[Cu(quin) $\left.)_{2}(1 \mathrm{a} 2 \mathrm{bOH})_{2}\right]$ (7a)}

A Teflon container was filled with $\left[\mathrm{Cu}(\text { quin })_{2}\left(\mathrm{H}_{2} \mathrm{O}\right)\right](50 \mathrm{mg}$, $0.12 \mathrm{mmol})$, acetonitrile $(7.5 \mathrm{~mL})$, triethylamine $(0.25 \mathrm{~mL})$ and 1-amino-2-butanol $(0.5 \mathrm{~mL})$. The container was closed and inserted into a steel autoclave, which was heated for 24 hours at $105{ }^{\circ} \mathrm{C}$. Afterwards, the autoclave was taken out from the oven and left overnight to cool to room temperature. Green needle-like crystals of trans-[Cu(quin $\left.)_{2}(1 \mathrm{a} 2 \mathrm{bOH})_{2}\right](7 \mathbf{a})$ were filtered off. Yield: $49 \mathrm{mg}, 71 \%$. IR (ATR, $\mathrm{cm}^{-1}$ ): $3431 \mathrm{w}$, $3260 \mathrm{~m}, 3166 \mathrm{w}, 3078 \mathrm{w}, 3061 \mathrm{w}, 2970 \mathrm{w}, 2957 \mathrm{~m}, 2939 \mathrm{w}, 2874 \mathrm{w}$, $1594 \mathrm{~s}, 1562 \mathrm{~s}, 1549 \mathrm{~s}, 1506 \mathrm{~m}, 1461 \mathrm{~s}, 1430 \mathrm{w}, 1370 \mathrm{~s}, 1343 \mathrm{~s}, 1325 \mathrm{~s}$, $1281 \mathrm{~m}, 1265 \mathrm{~m}, 1216 \mathrm{w}, 1170 \mathrm{~s}, 1151 \mathrm{~m}, 1137 \mathrm{~m}, 1115 \mathrm{~m}, 1088 \mathrm{~m}$, $1075 \mathrm{~m}, 1062 \mathrm{~m}, 1033 \mathrm{~m}, 986 \mathrm{~m}, 961 \mathrm{~s}, 897 \mathrm{~m}, 879 \mathrm{~m}, 859 \mathrm{~m}, 840 \mathrm{w}$, 802s, 774vvs, 742s, 707m, 632s, 601vs, 551m, 521m, 496s, $480 \mathrm{~m}, 441 \mathrm{w}, 428 \mathrm{w}$. Elemental analysis calcd for $\mathrm{C}_{28} \mathrm{H}_{34} \mathrm{CuN}_{4} \mathrm{O}_{6}$ (\%): C, 57.38; H, 5.85; N, 9.56. Found (\%): C, 57.53; H, 5.74; $\mathrm{N}, 9.70$.

\section{$\left[\mathrm{Cu}(\mathbf{1 a} 2 \mathrm{bOH})_{3}\right](\text { quin })_{2}(7 \mathrm{e})$}

$\left.[\mathrm{Cu} \text { (quin) })_{2}\left(\mathrm{H}_{2} \mathrm{O}\right)\right](50 \mathrm{mg}, 0.12 \mathrm{mmol})$, acetonitrile $(7.5 \mathrm{~mL})$ and 1-amino-2-butanol $(0.5 \mathrm{~mL})$ were added to an Erlenmeyer flask. The flask was closed and left to stand under ambient conditions. On the following day, blue crystalline solid, $\left[\mathrm{Cu}(1 \mathrm{a} 2 \mathrm{bOH})_{3}\right](\text { quin })_{2}$ (7e), was filtered off and air-dried. Yield: $75 \mathrm{mg}, 95 \%$. Please note that the same product formed at $4{ }^{\circ} \mathrm{C}$. IR (ATR, cm ${ }^{-1}$ ): 3263m, 3212m, 3139m, 2964m, 2933m, 2875m, $1836 \mathrm{w}, 1613 \mathrm{~s}, 1594 \mathrm{~m}, 1578 \mathrm{~m}, 1550 \mathrm{~s}, 1500 \mathrm{~m}, 1462 \mathrm{~s}, 1425 \mathrm{~m}$, $1383 \mathrm{~s}, 1367 \mathrm{vs}, 1340 \mathrm{~s}, 1312 \mathrm{~m}, 1249 \mathrm{w}, 1215 \mathrm{w}, 1202 \mathrm{w}, 1170 \mathrm{~s}$, $1146 \mathrm{w}, 1110 \mathrm{~m}, 1097 \mathrm{~m}, 1053 \mathrm{~m}, 1038 \mathrm{~s}, 1019 \mathrm{~m}, 981 \mathrm{~s}, 955 \mathrm{~m}$, 919w, 890w, 881w, 856m, 809s, 779vvs, 743s, 700m, 645m, $627 \mathrm{~m}, 594 \mathrm{~s}, 556 \mathrm{~m}, 548 \mathrm{~m}, 521 \mathrm{~m}, 498 \mathrm{~m}, 478 \mathrm{~m}, 459 \mathrm{w}, 430 \mathrm{w}$. Elemental analysis calcd for $\mathrm{C}_{32} \mathrm{H}_{45} \mathrm{CuN}_{5} \mathrm{O}_{7}(\%): \mathrm{C}, 56.92 ; \mathrm{H}$, 6.72; N, 10.37. Found (\%): C, 56.53; H, 6.66; N, 10.48.

\section{Reactions with $2 \mathrm{a} 1 \mathrm{pOH}(8)$}

The reactions with 2-amino-1-propanol are not reproducible. Furthermore, mixtures of products can form. The procedures listed below yielded a certain product at least once.

\section{[Cu(quin) $\left.)_{2}(\mathbf{2 a 1} \mathrm{pOH})\right](8 \mathrm{~b})$}

A Teflon container was filled with $\left[\mathrm{Cu}(\text { quin })_{2}\left(\mathrm{H}_{2} \mathrm{O}\right)\right](50 \mathrm{mg}$, $0.12 \mathrm{mmol})$, acetonitrile $(7.5 \mathrm{~mL})$ and 2-amino-1-propanol $(0.15 \mathrm{~mL})$. The container was closed and inserted into a steel autoclave, which was heated for 24 hours at $105{ }^{\circ} \mathrm{C}$. Afterwards, the autoclave was taken out from the oven and left overnight to cool to room temperature. Crystalline solid, [Cu(quin) $\left.)_{2}(2 \mathrm{a} 1 \mathrm{pOH})\right]$ (8b), was filtered off and air-dried. Yield: $46 \mathrm{mg}, 81 \%$. IR (ATR, $\mathrm{cm}^{-1}$ ): 3376w, 3343w, 3226w, 3140w, 3102w, 3056w, 
3009w, 2957w, 2932w, 2877w, 1641s, 1592m, 1565m, 1512w, $1459 \mathrm{~s}, 1429 \mathrm{w}, 1361 \mathrm{vs}, 1342 \mathrm{~s}, 1297 \mathrm{~m}, 1275 \mathrm{w}, 1264 \mathrm{w}, 1251 \mathrm{w}$, $1217 \mathrm{~m}, 1183 \mathrm{~m}, 1173 \mathrm{~s}, 1149 \mathrm{~m}, 1114 \mathrm{~m}, 1053 \mathrm{~s}, 1039 \mathrm{~s}, 969 \mathrm{w}$, $958 \mathrm{w}, 919 \mathrm{w}, 899 \mathrm{~s}, 883 \mathrm{w}, 850 \mathrm{~m}, 842 \mathrm{~m}, 800 \mathrm{vs}, 781 \mathrm{vvs}, 678 \mathrm{~m}$, 640m, 629s, 606s, 558m, 521m, 498s, 486m, 479m, 461w, $431 \mathrm{~m}, 414 \mathrm{~m}$. Elemental analysis calcd for $\mathrm{C}_{23} \mathrm{H}_{21} \mathrm{CuN}_{3} \mathrm{O}_{5}(\%)$ : C, 57.20; H, 4.38; N, 8.70. Found (\%): C, 57.15; H, 4.23; $\mathrm{N}, 8.47$.

\section{$\left[\mathrm{Cu}(\text { quin })_{2}(2 \mathrm{a} 1 \mathrm{pOH})\right]_{n}(8 \mathrm{c})$}

$\left[\mathrm{Cu}(\text { quin })_{2}\left(\mathrm{H}_{2} \mathrm{O}\right)\right](50 \mathrm{mg}, 0.12 \mathrm{mmol})$, acetonitrile $(6.5 \mathrm{~mL})$, methanol $(1 \mathrm{~mL})$ and 2-amino-1-propanol $(0.25 \mathrm{~mL})$ were added to an Erlenmeyer flask. The mixture was stirred thoroughly until all the solid was consumed. The resulting blue solution was left to stand under ambient conditions. After a period of time, crystals of $\left[\mathrm{Cu}(\text { quin })_{2}(2 \mathrm{a} 1 \mathrm{pOH})\right]_{n}(\mathbf{8 c})$ and $s y n-\left[\mathrm{Cu}_{2} \text { (quin }\right)_{2}{ }^{-}$ $\left.(2 \mathrm{a} 1 \mathrm{pO})_{2}\right]$ (syn-8d) were obtained. IR (ATR, $\left.\mathrm{cm}^{-1}\right): 3302 \mathrm{w}$, $3214 w, 3133 w, 3099 w, 3052 w, 2987 w, 2973 w, 2953 w, 2907 w$, $2823 \mathrm{w}, 1641 \mathrm{~s}, 1624 \mathrm{~s}, 1592 \mathrm{~s}, 1564 \mathrm{~m}, 1505 \mathrm{w}, 1457 \mathrm{~m}, 1427 \mathrm{w}$, $1359 \mathrm{~s}, 1341 \mathrm{~s}, 1258 \mathrm{~m}, 1252 \mathrm{~m}, 1216 \mathrm{~m}, 1169 \mathrm{~m}, 1153 \mathrm{~m}, 1141 \mathrm{~m}$, $1119 \mathrm{~m}, 1076 \mathrm{~s}, 1060 \mathrm{~s}, 1021 \mathrm{w}, 964 \mathrm{~m}, 955 \mathrm{w}, 917 \mathrm{w}, 897 \mathrm{~m}, 880 \mathrm{~m}$, 852m, 801vs, 778vvs, 640s, 629s, 608s, 599s, 570m, 523m, $494 \mathrm{~m}, 428 \mathrm{~s}, 406 \mathrm{~m}$. Elemental analysis calcd for $\mathrm{C}_{23} \mathrm{H}_{21} \mathrm{CuN}_{3} \mathrm{O}_{5}$ (\%): C, 57.20; H, 4.38; N, 8.70. Found (\%): C, 57.02; H, 4.19; $\mathrm{N}, 8.66$.

\section{$\operatorname{syn}-\left[\mathrm{Cu}_{2}(\text { quin })_{2}(2 \mathrm{a} 1 \mathrm{pO})_{2}\right](s y n-8 \mathrm{~d})$}

$\left[\mathrm{Cu}(\text { quin })_{2}\left(\mathrm{H}_{2} \mathrm{O}\right)\right]$ (50 $\left.\mathrm{mg}, 0.12 \mathrm{mmol}\right)$, acetonitrile $(6 \mathrm{~mL})$, methanol $(1.5 \mathrm{~mL})$, triethylamine $(0.25 \mathrm{~mL})$ and 2-amino-1propanol $(0.25 \mathrm{~mL})$ were added to an Erlenmeyer flask. The mixture was stirred thoroughly until all the solid was consumed. The resulting blue solution was left to stand under ambient conditions. After a period of time, a small amount of crystals of $s y n$-[Cu${ }_{2}$ (quin $\left.)_{2}(2 \mathrm{a} 1 \mathrm{pO})_{2}\right]$ (syn-8d) was obtained. IR (ATR, $\mathrm{cm}^{-1}$ ): 3323w, 3251s, 3145m, 3066w, 3050w, 2955m, $2917 \mathrm{~m}, 2875 \mathrm{~m}, 2841 \mathrm{w}, 2804 \mathrm{w}, 2711 \mathrm{w}, 1635 \mathrm{vvs}, 1596 \mathrm{~m}, 1567 \mathrm{~s}$, $1556 \mathrm{~m}, 1508 \mathrm{~m}, 1462 \mathrm{~m}, 1431 \mathrm{w}, 1352 \mathrm{vs}, 1304 \mathrm{~m}, 1276 \mathrm{~m}, 1262 \mathrm{w}$, $1216 \mathrm{w}, 1204 \mathrm{w}, 1175 \mathrm{~s}, 1148 \mathrm{~m}, 1116 \mathrm{~m}, 1070 \mathrm{~s}, 1047 \mathrm{~s}, 1020 \mathrm{~m}$, 966m, 955w, 938w, 922w, 896m, 876m, 853s, 801vs, 771vvs, $742 \mathrm{~s}, 716 \mathrm{~s}, 661 \mathrm{w}, 634 \mathrm{~s}, 626 \mathrm{~m}, 603 \mathrm{~s}, 574 \mathrm{~m}, 556 \mathrm{~m}, 535 \mathrm{~m}, 521 \mathrm{~m}$, $495 \mathrm{~s}, 457 \mathrm{~s}, 428 \mathrm{~s}$. Elemental analysis calcd for $\mathrm{C}_{26} \mathrm{H}_{28} \mathrm{Cu}_{2} \mathrm{~N}_{4} \mathrm{O}_{6}$ (\%): C, 50.40; H, 4.56; N, 9.04. Found (\%): C, 50.28; H, 4.56; N, 8.94.

\section{$\left[\mathrm{Cu}(2 \mathrm{a} 1 \mathrm{pOH})_{3}\right](\text { quin })_{2}(8 \mathrm{e})$}

Procedure A. [Cu(quin $\left.)_{2}\left(\mathrm{H}_{2} \mathrm{O}\right)\right](50 \mathrm{mg}, 0.12 \mathrm{mmol})$, acetonitrile $(7.5 \mathrm{~mL})$ and 2-amino-1-propanol $(0.25 \mathrm{~mL})$ were added to an Erlenmeyer flask. The flask was closed and left to stand under ambient conditions. On the following day, blue crystalline solid, $\left[\mathrm{Cu}(2 \mathrm{a} 1 \mathrm{pOH})_{3}\right](\text { quin })_{2}$ (8e), was filtered off and airdried. Yield: $58 \mathrm{mg}, 78 \%$. Procedure B. A Teflon container was filled with $\left.[\mathrm{Cu} \text { (quin })_{2}\left(\mathrm{H}_{2} \mathrm{O}\right)\right](50 \mathrm{mg}, 0.12 \mathrm{mmol})$, acetonitrile $(7.5 \mathrm{~mL})$ and 2-amino-1-propanol $(0.5 \mathrm{~mL})$. The container was closed and inserted into a steel autoclave, which was heated for 24 hours at $105{ }^{\circ} \mathrm{C}$. Afterwards, the autoclave was taken out from the oven and left overnight to cool to room temperature.
The resulting green solution was stored at $4{ }^{\circ} \mathrm{C}$. Crystals of $8 \mathbf{e}$ were filtered off after a few days. Yield: $46 \mathrm{mg}, 62 \%$. IR (ATR, $\mathrm{cm}^{-1}$ ): 3213m, 3123m, 2962w, 2926w, 2881w, 1835w, 1606s, $1557 \mathrm{~s}, 1500 \mathrm{~m}, 1460 \mathrm{~m}, 1424 \mathrm{~m}, 1365 \mathrm{vs}, 1337 \mathrm{~s}, 1296 \mathrm{~m}, 1245 \mathrm{w}$, $1212 \mathrm{~m}, 1199 \mathrm{w}, 1167 \mathrm{~m}, 1143 \mathrm{w}, 1109 \mathrm{~s}, 1070 \mathrm{~m}, 1043 \mathrm{~s}, 989 \mathrm{w}$, 955m, 919w, 890m, 878m, 855m, 838w, 806s, 778vvs, 740s, $680 \mathrm{~m}, 656 \mathrm{~m}, 627 \mathrm{~s}, 593 \mathrm{~s}, 552 \mathrm{~m}, 521 \mathrm{~m}, 476 \mathrm{~m}, 455 \mathrm{~m}, 406 \mathrm{w}$. Elemental analysis calcd for $\mathrm{C}_{29} \mathrm{H}_{39} \mathrm{CuN}_{5} \mathrm{O}_{7}(\%): \mathrm{C}, 55.01 ; \mathrm{H}$, 6.21; N, 11.06. Found (\%): C, 54.78; H, 6.12; N, 10.73.

\section{$\operatorname{syn}-\left[\mathrm{Cu}_{2}(\mathrm{quin})_{2}(2 \mathrm{a} 2 \mathrm{~m} 1 \mathrm{pO})_{2}\right](\operatorname{syn}-9 \mathrm{~d})$}

$\left[\mathrm{Cu}(\text { quin })_{2}\left(\mathrm{H}_{2} \mathrm{O}\right)\right]$ (50 $\left.\mathrm{mg}, 0.12 \mathrm{mmol}\right)$, acetonitrile $(6 \mathrm{~mL})$, methanol $(1.5 \mathrm{~mL})$ and 2-amino-2-methyl-1-propanol $(0.5 \mathrm{~mL})$ were added to an Erlenmeyer flask. The mixture was stirred thoroughly until all the solid was consumed. The resulting blue solution was left to stand under ambient conditions. After a few days, a small amount of crystalline $s y n$ - $\left.\left[\mathrm{Cu}_{2} \text { (quin }\right)_{2}(2 \mathrm{a} 2 \mathrm{~m} 1 \mathrm{pO})_{2}\right]$ (syn-9d) was obtained. IR (ATR, $\mathrm{cm}^{-1}$ ): $3255 \mathrm{~m}, 3145 \mathrm{~m}, 3060 \mathrm{w}$, 2971 w, 2951w, 2905w, 2871w, 2835w, 1638vvs, 1597m, 1567m, $1506 \mathrm{w}, 1462 \mathrm{~m}, 1361 \mathrm{vs}, 1302 \mathrm{w}, 1255 \mathrm{w}, 1231 \mathrm{~m}, 1188 \mathrm{~m}, 1172 \mathrm{~m}$, $1148 \mathrm{~m}, 1129 \mathrm{~m}, 1068 \mathrm{~m}, 1047 \mathrm{~s}, 1019 \mathrm{~m}, 983 \mathrm{~m}, 958 \mathrm{w}, 924 \mathrm{w}$, $897 \mathrm{~m}, 875 \mathrm{w}, 853 \mathrm{~s}, 804 \mathrm{~s}, 772 \mathrm{vs}, 738 \mathrm{~m}, 707 \mathrm{~m}, 627 \mathrm{~s}, 602 \mathrm{~m}$, $545 \mathrm{~m}, 522 \mathrm{~m}, 499 \mathrm{~m}, 444 \mathrm{~s}, 413 \mathrm{~s}$. Elemental analysis calcd for $\mathrm{C}_{28} \mathrm{H}_{32} \mathrm{Cu}_{2} \mathrm{~N}_{4} \mathrm{O}_{6}$ (\%): C, 51.93; H, 4.98; N, 8.65. Found (\%): C, 51.89; H, 5.06; N, 8.88.

\section{$\left[\mathrm{Cu}(2 \mathrm{a} 2 \mathrm{~m} 1 \mathrm{pO})_{2}\right](2 \mathrm{a} 2 \mathrm{~m} 1 \mathrm{pOHH})_{2}(\text { quin })_{2}$ (9f)}

Procedure A. $\left[\mathrm{Cu}(\text { quin })_{2}\left(\mathrm{H}_{2} \mathrm{O}\right)\right](50 \mathrm{mg}, 0.12 \mathrm{mmol})$, acetonitrile $(7.5 \mathrm{~mL})$ and 2-amino-2-methyl-1-propanol $(0.5 \mathrm{~mL})$ were added to an Erlenmeyer flask. The flask was closed and left to stand under ambient conditions. On the following day, a mixture of blue crystalline solid, syn-9d, and colorless needle-like crystals of $2 \mathrm{a} 2 \mathrm{~m} 1 \mathrm{pOHH}^{+}$guin $^{-}\left(2 \mathrm{a} 2 \mathrm{~m} 1 \mathrm{pOHH}^{+}=\mathrm{a}\right.$ cationic form of 2-amino-2-methyl-1-propanol with a $\mathrm{NH}_{3}{ }^{+}$moiety) was obtained. $2 \mathrm{a} 2 \mathrm{~m} 1 \mathrm{pOHH}^{+}$quin $^{-}$crystallized in two polymorphic modifications. After a few days, only purple crystals of $\left[\mathrm{Cu}(2 \mathrm{a} 2 \mathrm{~m} 1 \mathrm{pO})_{2}\right]$ $(2 \mathrm{a} 2 \mathrm{~m} 1 \mathrm{pOHH})_{2}$ (quin $)_{2}$ (9f) were left in the flask. The purple solid was filtered off. Yield: $83 \mathrm{mg}, 92 \%$. Procedure B. A Teflon container was filled with $\left[\mathrm{Cu}(\text { quin })_{2}\left(\mathrm{H}_{2} \mathrm{O}\right)\right](50 \mathrm{mg}, 0.12 \mathrm{mmol})$, acetonitrile (6 mL), methanol (1.5 mL) and 2-amino-2-methyl-1-propanol $(0.5 \mathrm{~mL})$. The container was closed and inserted into a steel autoclave, which was heated for 24 hours at $105{ }^{\circ} \mathrm{C}$. Afterwards, the autoclave was taken out from the oven and left overnight to cool to room temperature. The resulting green solution was concentrated under reduced pressure on a rotary evaporator. A glass vial with diethyl ether was carefully inserted into the Erlenmeyer flask with the concentrate. After a few days, purple crystals of 9f grew from the solution. Yield: $73 \mathrm{mg}, 81 \%$. IR (ATR, $\mathrm{cm}^{-1}$ ): 3315m, 3213w, 3076m, 2982m, 2957m, 2869m, $2845 \mathrm{~m}, 2792 \mathrm{~m}, 2743 \mathrm{~m}, 2610 \mathrm{~m}, 2550 \mathrm{~m}, 2512 \mathrm{~m}, 2110 \mathrm{w}, 1644 \mathrm{~m}$, $1602 \mathrm{~s}, 1582 \mathrm{~s}, 1553 \mathrm{~s}, 1501 \mathrm{~m}, 1471 \mathrm{~m}, 1464 \mathrm{~m}, 1423 \mathrm{w}, 1384 \mathrm{~s}$, $1372 \mathrm{vs}, 1326 \mathrm{w}, 1297 \mathrm{w}, 1275 \mathrm{~m}, 1260 \mathrm{w}, 1222 \mathrm{w}, 1211 \mathrm{w}, 1190 \mathrm{~m}$, $1169 \mathrm{~m}, 1147 \mathrm{~m}, 1100 \mathrm{w}, 1055 \mathrm{vvs}, 1010 \mathrm{~s}, 984 \mathrm{~m}, 975 \mathrm{~m}, 954 \mathrm{w}$, $928 \mathrm{w}, 913 \mathrm{w}, 903 \mathrm{w}, 890 \mathrm{~m}, 870 \mathrm{w}, 852 \mathrm{~m}, 802 \mathrm{~s}, 786 \mathrm{vvs}, 775 \mathrm{vvs}$, $738 \mathrm{~m}, 690 \mathrm{~m}, 637 \mathrm{w}, 626 \mathrm{~s}, 608 \mathrm{~s}, 593 \mathrm{~m}, 564 \mathrm{w}, 521 \mathrm{~m}, 474 \mathrm{~s}, 458 \mathrm{~s}$, 
442s, 422m. Elemental analysis calcd for $\mathrm{C}_{36} \mathrm{H}_{56} \mathrm{CuN}_{6} \mathrm{O}_{8}(\%): \mathrm{C}$, 56.56; H, 7.38; N, 10.99. Found (\%): C, 56.50; H, 7.43; N, 10.98.

\section{$\left.\operatorname{syn}-\left[\mathrm{Cu}_{2} \text { (quin }\right)_{2}(2 \mathrm{a} 1 \mathrm{bO})_{2}\right]($ syn-10d)}

$\left[\mathrm{Cu}(\text { quin })_{2}\left(\mathrm{H}_{2} \mathrm{O}\right)\right](50 \mathrm{mg}, 0.12 \mathrm{mmol})$, acetonitrile $(7.5 \mathrm{~mL})$ and 2-amino-1-butanol $(0.25 \mathrm{~mL})$ were added to an Erlenmeyer flask. The flask was closed and left to stand under ambient conditions. On the following day, a small amount of crystalline solid, syn- $\left[\mathrm{Cu}_{2}(\text { quin })_{2}(2 \mathrm{a} 1 \mathrm{bO})_{2}\right]$ (syn-10d), was filtered off and air-dried. Note. On prolonged standing in solution, the salt, $2 \mathrm{a} 1 \mathrm{bOHH}^{+}$quin $^{-}\left(2 \mathrm{a} 1 \mathrm{bOHH}^{+}=\right.$a cationic form of 2-amino-1butanol with a $\mathrm{NH}_{3}{ }^{+}$moiety), precipitated. $2 \mathrm{a} 1 \mathrm{bOHH}^{+}$quin ${ }^{-}$ crystallized in two polymorphic modifications. IR (ATR, $\mathrm{cm}^{-1}$ ): $3255 \mathrm{~s}, 3143 \mathrm{~m}, 3051 \mathrm{w}, 2965 \mathrm{~m}, 2920 \mathrm{~m}, 2896 \mathrm{~m}, 2880 \mathrm{~m}, 2837 \mathrm{~m}$, 2806w, 2701w, 1636vvs, $1594 \mathrm{~m}, 1567 \mathrm{~s}, 1506 \mathrm{~m}, 1460 \mathrm{~s}, 1351 \mathrm{vs}$, $1261 \mathrm{w}, 1214 \mathrm{w}, 1172 \mathrm{~s}, 1147 \mathrm{~m}, 1128 \mathrm{~m}, 1105 \mathrm{~m}, 1075 \mathrm{~s}, 1019 \mathrm{~m}$, 987m, 964m, 954w, 930w, 896m, 874m, 867w, 851s, 801s, $770 \mathrm{vs,}$ 739s, 719s, 634s, 602s, 555m, 538m, 521m, 493s, 467m, 434s. Elemental analysis calcd for $\mathrm{C}_{28} \mathrm{H}_{32} \mathrm{Cu}_{2} \mathrm{~N}_{4} \mathrm{O}_{6}$ (\%): C, 51.93; H, 4.98; N, 8.65. Found (\%): C, 51.31; H, 4.58; N, 8.70.

\section{$\left[\mathrm{Cu}(\mathbf{2 a 1 b O H})_{3}\right](\text { quin })_{2}(\mathbf{1 0 e})$}

$\left[\mathrm{Cu}(\text { quin })_{2}\left(\mathrm{H}_{2} \mathrm{O}\right)\right](50 \mathrm{mg}, 0.12 \mathrm{mmol})$, acetonitrile $(6 \mathrm{~mL})$, methanol $(1.5 \mathrm{~mL})$ and 2-amino-1-butanol $(0.5 \mathrm{~mL})$ were added to an Erlenmeyer flask. The mixture was stirred thoroughly until all the solid was consumed. The resulting blue solution was left to stand under ambient conditions. On the following day, the solution was concentrated under reduced pressure on a rotary evaporator. A glass vial with diethyl ether was carefully inserted into the Erlenmeyer flask with the concentrate. Typically, a mixture of blue crystals of $\left[\mathrm{Cu}(2 \mathrm{a} 1 \mathrm{bOH})_{3}\right](\text { quin })_{2}(\mathbf{1 0 e})$, syn-10d and/or $2 \mathrm{a} 1 \mathrm{bOHH}^{+}$quin $^{-}$was obtained. IR (ATR, $\left.\mathrm{cm}^{-1}\right)$ : $3218 \mathrm{~m}, 3125 \mathrm{~m}, 2963 \mathrm{~m}, 2934 \mathrm{~m}, 2877 \mathrm{w}, 1840 \mathrm{w}, 1594 \mathrm{~s}, 1557 \mathrm{~s}$, $1500 \mathrm{~m}, 1460 \mathrm{~s}, 1423 \mathrm{~m}, 1366 \mathrm{vs}, 1337 \mathrm{~s}, 1247 \mathrm{w}, 1211 \mathrm{w}, 1199 \mathrm{w}$, $1167 \mathrm{~s}, 1144 \mathrm{~m}, 1108 \mathrm{~m}, 1095 \mathrm{~m}, 1044 \mathrm{vs}, 997 \mathrm{~m}, 953 \mathrm{~m}, 922 \mathrm{w}$, $889 \mathrm{~m}, 880 \mathrm{~m}, 854 \mathrm{~s}, 806 \mathrm{~s}, 778 \mathrm{vvs}, 741 \mathrm{~s}, 626 \mathrm{~s}, 591 \mathrm{~s}, 550 \mathrm{w}$, $521 \mathrm{~m}, 486 \mathrm{~m}, 477 \mathrm{~m}, 437 \mathrm{w}$. Elemental analysis calcd for $\mathrm{C}_{32} \mathrm{H}_{45} \mathrm{CuN}_{5} \mathrm{O}_{7}$ (\%): C, 56.92; H, 6.72; N, 10.37. Found (\%): C, 56.74; H, 6.74; N, 10.21 .

\section{trans $-\left[\mathrm{Cu}(\text { quin })_{2}(3 \mathrm{a} 1 \mathrm{pOH})_{2}\right](11 \mathrm{a})$}

$\left[\mathrm{Cu}(\text { quin })_{2}\left(\mathrm{H}_{2} \mathrm{O}\right)\right](50 \mathrm{mg}, 0.12 \mathrm{mmol})$, acetonitrile $(7.5 \mathrm{~mL})$ and 3-amino-1-propanol $(150 \mathrm{mg})$ were added to an Erlenmeyer flask. The flask was closed and left to stand under ambient conditions. On the following day, blue needle-like crystals of trans-[Cu(quin $\left.)_{2}(3 \mathrm{a} 1 \mathrm{pOH})_{2}\right]$ (11a) were filtered off. Yield: $58 \mathrm{mg}$, $89 \%$. IR (ATR, $\mathrm{cm}^{-1}$ ): 3384m, 3329m, 3224m, 3052w, 2959w, 2943w, 2914w, 2869w, 2858w, 2815w, 1623s, 1595m, 1559m, $1548 \mathrm{~m}, 1504 \mathrm{w}, 1480 \mathrm{w}, 1459 \mathrm{~m}, 1432 \mathrm{~m}, 1408 \mathrm{w}, 1385 \mathrm{~s}, 1371 \mathrm{vs}$, $1342 \mathrm{~s}, 1263 \mathrm{~m}, 1213 \mathrm{~m}, 1174 \mathrm{~m}, 1161 \mathrm{~m}, 1146 \mathrm{~m}, 1051 \mathrm{~s}, 1030 \mathrm{~m}$, $1021 \mathrm{~m}, 995 \mathrm{~s}, 983 \mathrm{~m}, 961 \mathrm{~m}, 899 \mathrm{~s}, 875 \mathrm{w}, 855 \mathrm{~m}, 810 \mathrm{~s}, 802 \mathrm{~s}$, 773vvs, 738s, 629vs, 618s, 600s, 560m, 520s, 495s, 476m, $458 \mathrm{~m}$. Elemental analysis calcd for $\mathrm{C}_{26} \mathrm{H}_{30} \mathrm{CuN}_{4} \mathrm{O}_{6}$ (\%): C, 55.96; H, 5.42; N, 10.04. Found (\%): C, 55.68; H, 5.23; N, 10.11.

\section{trans-[Cu(quin $\left.)_{2}(3 \mathrm{a} 1 \mathrm{pOH})_{2}\right] \cdot 3 \mathrm{a} 1 \mathrm{pOH}(11 \mathrm{a} \cdot 3 \mathrm{a} 1 \mathrm{pOH})$}

$\left[\mathrm{Cu}(\text { quin })_{2}\left(\mathrm{H}_{2} \mathrm{O}\right)\right](25 \mathrm{mg}, 0.06 \mathrm{mmol})$ and acetonitrile $(7.5 \mathrm{~mL})$ were added to an Erlenmeyer flask. After the contents were cooled to $4{ }^{\circ} \mathrm{C}$, 3-amino-1-propanol $(150 \mathrm{mg})$ was added. The reaction mixture was left to stand in a closed vessel at $4{ }^{\circ} \mathrm{C}$ for approximately 1 hour. Afterwards, blue crystalline solid, trans$\left[\mathrm{Cu}(\text { quin })_{2}(3 \mathrm{a} 1 \mathrm{pOH})_{2}\right] \cdot 3 \mathrm{a} 1 \mathrm{pOH}(\mathbf{1 1 a} \cdot \mathbf{3 a 1} \mathbf{p O H})$, was filtered off. Yield: $29 \mathrm{mg}$. Please note that owing to the solvent molecules of $3 \mathrm{a} 1 \mathrm{pOH}$, the crystals of 11a.3a1pOH are not stable when taken out from the mother liquor. In the solution, 11a.3a1pOH converts into 11a within a day. IR (ATR, $\mathrm{cm}^{-1}$ ): $3359 \mathrm{w}$, $3255 \mathrm{~m}, 3175 \mathrm{~m}, 3050 \mathrm{w}, 2950 \mathrm{w}, 2867 \mathrm{w}, 1634 \mathrm{~s}, 1614 \mathrm{~s}, 1588 \mathrm{~s}$, $1562 \mathrm{~s}, 1504 \mathrm{~m}, 1486 \mathrm{w}, 1462 \mathrm{~m}, 1427 \mathrm{w}, 1391 \mathrm{~s}, 1377 \mathrm{~s}, 1359 \mathrm{~s}$, $1344 \mathrm{~s}, 1299 \mathrm{w}, 1268 \mathrm{w}, 1215 \mathrm{w}, 1168 \mathrm{~m}, 1153 \mathrm{~m}, 1147 \mathrm{~m}, 1109 \mathrm{w}$, $1060 \mathrm{~s}, 1044 \mathrm{~s}, 1017 \mathrm{~m}, 993 \mathrm{~m}, 955 \mathrm{w}, 931 \mathrm{~m}, 894 \mathrm{~s}, 855 \mathrm{~m}, 849 \mathrm{~m}$, $801 \mathrm{vs}, 775 \mathrm{vvs}, 745 \mathrm{~m}, 672 \mathrm{~m}, 630 \mathrm{~s}, 599 \mathrm{~s}, 550 \mathrm{~m}, 521 \mathrm{~m}$, 500m, 482w.

\section{$\left[\mathrm{Cu}(\text { quin })_{2}(3 \mathrm{a} 1 \mathrm{pOH})\right](\mathbf{1 1 b})$}

A Teflon container was filled with $\left[\mathrm{Cu}(\text { quin })_{2}\left(\mathrm{H}_{2} \mathrm{O}\right)\right](50 \mathrm{mg}$, $0.12 \mathrm{mmol})$, acetonitrile $(7.5 \mathrm{~mL})$ and 3-amino-1-propanol $(150 \mathrm{mg})$. The container was closed and inserted into a steel autoclave, which was heated for 24 hours at $105^{\circ} \mathrm{C}$. Afterwards, the autoclave was taken out from the oven and left overnight to cool to room temperature. The resulting green solution was transferred to an Erlenmeyer flask which was left open for 1 day. Afterwards, the flask was stoppered. Within 24 hours, crystals of $\left[\mathrm{Cu}(\text { quin })_{2}(3 \mathrm{a} 1 \mathrm{pOH})\right]$ (11b) grew from the oil. Please note that 11b was obtained as a one-time event, otherwise 11a, 11a.3a1pOH or anti-11d.2MeCN formed. IR (ATR, $\mathrm{cm}^{-1}$ ): 3345m, 3233m, 3154m, 3104w, 3056w, 3011w, 2985w, 2943w, 2917w, 2879w, 1641s, 1594s, 1564s, 1506m, 1458s, 1435w, $1416 \mathrm{w}, 1362 \mathrm{vs}, 1342 \mathrm{~s}, 1295 \mathrm{~m}, 1259 \mathrm{~m}, 1242 \mathrm{~m}, 1217 \mathrm{~m}, 1183 \mathrm{~m}$, $1173 \mathrm{~s}, 1151 \mathrm{~m}, 1112 \mathrm{~s}, 1093 \mathrm{~m}, 1046 \mathrm{~m}, 1024 \mathrm{w}, 975 \mathrm{~s}, 958 \mathrm{~m}, 946 \mathrm{~s}$, 899s, 881m, 851s, 833w, 799s, 782vvs, 661s, 640s, 633s, 627s, $606 \mathrm{~s}, 555 \mathrm{~m}, 521 \mathrm{~s}, 497 \mathrm{~s}, 478 \mathrm{~m}$.

\section{$\left[\mathrm{Cu}(\text { quin })_{2}(3 \mathrm{a} 1 \mathrm{pOH})\right]_{n}(\mathbf{1 1 c})$}

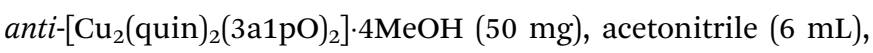
methanol $(1.5 \mathrm{~mL})$, quinaldinic acid $(28 \mathrm{mg})$ and 3-amino-1propanol $(24 \mathrm{mg})$ were added to an Erlenmeyer flask. The contents were stirred at room temperature for 1 day. All the solid was consumed meanwhile. The resulting blue solution was stored at $4{ }^{\circ} \mathrm{C}$ for a few days. Crystals of $\left[\mathrm{Cu}(\text { quin })_{2}-\right.$ $(3 \mathrm{a} 1 \mathrm{pOH})]_{n}(\mathbf{1 1 c})$ were filtered off and air-dried. Yield: $22 \mathrm{mg}$. IR (ATR, $\mathrm{cm}^{-1}$ ): 3335w, 3238w, 3154w, 3095w, 3071w, 2953w, 2928w, 2886w, 1644s, 1628s, 1598s, 1564m, 1506w, $1475 \mathrm{w}, 1460 \mathrm{~s}, 1446 \mathrm{w}, 1360 \mathrm{vs}, 1343 \mathrm{~s}, 1295 \mathrm{w}, 1257 \mathrm{~m}, 1237 \mathrm{w}$, $1217 \mathrm{w}, 1197 \mathrm{w}, 1180 \mathrm{~m}, 1172 \mathrm{~m}, 1154 \mathrm{~m}, 1139 \mathrm{~m}, 1081 \mathrm{~m}, 1066 \mathrm{~s}$, $1034 \mathrm{~m}, 994 \mathrm{w}, 963 \mathrm{w}, 956 \mathrm{w}, 901 \mathrm{~m}, 881 \mathrm{~m}, 858 \mathrm{~m}, 816 \mathrm{~m}, 803 \mathrm{vs}$, $775 \mathrm{vvs}, 750 \mathrm{~m}, 640 \mathrm{~m}, 630 \mathrm{~m}, 608 \mathrm{~s}, 600 \mathrm{~m}, 569 \mathrm{w}, 548 \mathrm{w}, 530 \mathrm{w}$, $521 \mathrm{~m}, 499 \mathrm{~m}, 431 \mathrm{~m}, 405 \mathrm{~m}$. Elemental analysis calcd for $\mathrm{C}_{23} \mathrm{H}_{21} \mathrm{CuN}_{3} \mathrm{O}_{5}(\%):$ C, 57.20; H, 4.38; N, 8.70. Found (\%): C, $57.18 ; \mathrm{H}, 4.30 ; \mathrm{N}, 8.16$. 


\section{$\operatorname{syn}$-[ $\left.\mathrm{Cu}_{2}(\text { quin })_{2}(3 \mathrm{a} 1 \mathrm{pO})_{2}\right]($ syn-11d)}

A Teflon container was filled with $\left[\mathrm{Cu}(\text { quin })_{2}\left(\mathrm{H}_{2} \mathrm{O}\right)\right](50 \mathrm{mg}$, $0.12 \mathrm{mmol})$, acetonitrile $(7.5 \mathrm{~mL})$ and 3-amino-1-propanol $(150 \mathrm{mg})$. The container was closed and inserted into a steel autoclave, which was heated for 24 hours at $105{ }^{\circ} \mathrm{C}$. Afterwards, the autoclave was taken out from the oven and left overnight to cool to room temperature. The resulting green solution was concentrated under reduced pressure on a rotary evaporator. A glass vial with diethyl ether was carefully inserted into the Erlenmeyer flask with the concentrate. After a period of time, crystals of syn-[ $\mathrm{Cu}_{2}$ (quin $\left.)_{2}(3 \mathrm{a} 1 \mathrm{pO})_{2}\right]$ (syn-11d) and 11a deposited from the concentrate. Please note that syn-11d was obtained only once.

\section{$\operatorname{syn}-\left[\mathrm{Cu}_{2}(\text { quin })_{2}(3 \mathrm{a} 1 \mathrm{pO})_{2}\right] \cdot 2 \mathrm{H}_{2} \mathrm{O}\left(\operatorname{syn}-11 \mathrm{~d} \cdot 2 \mathrm{H}_{2} \mathrm{O}\right)$}

A Teflon container was filled with $\left[\mathrm{Cu}(\text { quin })_{2}\left(\mathrm{H}_{2} \mathrm{O}\right)\right](50 \mathrm{mg}$, $0.12 \mathrm{mmol})$, methanol $(7.5 \mathrm{~mL})$, triethylamine $(0.5 \mathrm{~mL})$ and 3-amino-1-propanol $(150 \mathrm{mg})$. The container was closed and inserted into a steel autoclave, which was heated for 24 hours at $105{ }^{\circ} \mathrm{C}$. Afterwards, the autoclave was taken out from the oven and left overnight to cool to room temperature. The resulting green solution was concentrated under reduced pressure on a rotary evaporator. A glass vial with diethyl ether was carefully inserted into the Erlenmeyer flask with the concentrate. After a period of time, crystals of $\left.s y n-\left[\mathrm{Cu}_{2} \text { (quin }\right)_{2}(3 \mathrm{a} 1 \mathrm{pO})_{2}\right] \cdot 2 \mathrm{H}_{2} \mathrm{O}$ (syn11d $2 \mathrm{H}_{2} \mathrm{O}$ ), syn-11d and 11a deposited from the concentrate. Please note that a more reliable way of preparing $\boldsymbol{s y n}-\mathbf{1 1 d} \cdot 2 \mathrm{H}_{2} \mathrm{O}$ proved to be by powdering a sample of anti-11d-4MeOH. The powdered material was left to stand in the air for at least 1 day. IR (ATR, $\mathrm{cm}^{-1}$ ): 3469m, 3271m, 3160m, 3058w, 2936w, 2906w, $2885 \mathrm{w}, 2806 \mathrm{~m}, 2698 \mathrm{w}, 1633 \mathrm{vs}, 1615 \mathrm{vs}, 1594 \mathrm{~s}, 1563 \mathrm{~s}, 1506 \mathrm{~m}$, $1460 \mathrm{~s}, 1429 \mathrm{w}, 1372 \mathrm{vs}, 1343 \mathrm{~s}, 1328 \mathrm{~m}, 1306 \mathrm{w}, 1277 \mathrm{w}, 1260 \mathrm{w}$, $1215 \mathrm{w}, 1180 \mathrm{~s}, 1172 \mathrm{~s}, 1148 \mathrm{~m}, 1106 \mathrm{w}, 1085 \mathrm{~s}, 1055 \mathrm{~s}, 1017 \mathrm{w}$, 985w, 956w, 932s, 896m, 883w, 877m, 852m, 802s, 778vvs, $741 \mathrm{~m}, 718 \mathrm{~m}, 658 \mathrm{~m}, 642 \mathrm{~m}, 633 \mathrm{~m}, 596 \mathrm{~s}, 520 \mathrm{~s}, 494 \mathrm{~s}, 450 \mathrm{~s}$. Elemental analysis calcd for $\mathrm{C}_{26} \mathrm{H}_{32} \mathrm{Cu}_{2} \mathrm{~N}_{4} \mathrm{O}_{8}(\%)$ : C, 47.63; H, 4.92; N, 8.55. Found (\%): C, 47.54; H, 4.72; N, 8.16.

\section{anti-[ $\mathrm{Cu}_{2}$ (quin) $\left.)_{2}(3 \mathrm{a} 1 \mathrm{pO})_{2}\right] \cdot 2 \mathrm{MeCN}($ anti-11d·2MeCN)}

Procedure $A$. [Cu(quin) $\left.)_{2}\left(\mathrm{H}_{2} \mathrm{O}\right)\right](50 \mathrm{mg}, 0.12 \mathrm{mmol})$, acetonitrile $(6 \mathrm{~mL})$ and methanol $(1.5 \mathrm{~mL})$ were added to an Erlenmeyer flask. After, the contents were cooled to $4{ }^{\circ} \mathrm{C}$, 3-amino-1propanol $(150 \mathrm{mg})$ was added. The reaction mixture was left to stand in a closed vessel at $4{ }^{\circ} \mathrm{C}$. Blue-green crystals of anti$\left[\mathrm{Cu}_{2}(\text { quin })_{2}(3 \mathrm{a} 1 \mathrm{pO})_{2}\right] \cdot 2 \mathrm{MeCN}(\boldsymbol{a n t i - 1 1 d} \cdot \mathbf{2 M e C N})$ were obtained after a few days. Procedure B. A Teflon container was filled with $\left.[\mathrm{Cu} \text { (quin) })_{2}\left(\mathrm{H}_{2} \mathrm{O}\right)\right](50 \mathrm{mg}, 0.12 \mathrm{mmol})$, acetonitrile $(7.5 \mathrm{~mL})$, triethylamine $(0.5 \mathrm{~mL})$ and 3-amino-1-propanol $(150 \mathrm{mg})$. The container was closed and inserted into a steel autoclave, which was heated for 24 hours at $105{ }^{\circ} \mathrm{C}$. Afterwards, the autoclave was taken out from the oven and left overnight to cool to room temperature. The resulting green solution was concentrated under reduced pressure on a rotary evaporator. The concentrate was transferred to an Erlenmeyer flask and left to stand under ambient conditions. Crystals of anti-11d-2MeCN were obtained after few days. Please note that crystals of anti-11d-2MeCN are not stable when taken out from the mother liquor. IR (ATR, $\mathrm{cm}^{-1}$ ): 3310m, 3219w, 3141w, 3056w, 3023w, 2983w, 2931w, 2876m, 2823w, 2708w, 2249w, 1630vs, 1619s, 1593m, 1560s, $1507 \mathrm{~m}, 1461 \mathrm{~m}, 1430 \mathrm{~m}, 1397 \mathrm{w}, 1359 \mathrm{vs}, 1343 \mathrm{~s}, 1301 \mathrm{~m}, 1275 \mathrm{~m}$, $1260 \mathrm{~m}, 1213 \mathrm{w}, 1178 \mathrm{~m}, 1149 \mathrm{~s}, 1112 \mathrm{w}, 1082 \mathrm{~s}, 1059 \mathrm{~s}, 1041 \mathrm{~m}$, 1021w, 969w, 956w, 930s, 885s, 858w, 821w, 811m, 779vvs, 745s, $684 \mathrm{~m}, 641 \mathrm{~m}, 633 \mathrm{~m}, 596 \mathrm{~s}, 521 \mathrm{~m}, 501 \mathrm{~m}, 486 \mathrm{~m}, 434 \mathrm{~m}, 407 \mathrm{~m}$.

\section{anti-[ $\mathrm{Cu}_{2}$ (quin) $\left.)_{2}(3 \mathrm{a} 1 \mathrm{pO})_{2}\right] \cdot 4 \mathrm{MeOH}($ anti-11d·4MeOH)}

$\left[\mathrm{Cu}(\text { quin })_{2}\left(\mathrm{H}_{2} \mathrm{O}\right)\right](200 \mathrm{mg}, 0.47 \mathrm{mmol})$, methanol $(7.5 \mathrm{~mL})$ and 3-amino-1-propanol $(300 \mathrm{mg}$ ) were added to an Erlenmeyer flask. The flask was closed and left to stand under ambient conditions. After a few minutes, all the solid was consumed and the solution acquired a green color. Large, dark blue crystals of anti-[ $\left.\mathrm{Cu}_{2}(\text { quin })_{2}(3 \mathrm{a} 1 \mathrm{pO})_{2}\right] \cdot 4 \mathrm{MeOH}$ (anti-11d-4MeOH) grew overnight. Yield: $141 \mathrm{mg}$. Please note that the crystals of anti11d.4MeOH are not stable when removed from the mother liquor. In the air, they are converted into $\mathbf{s y n}-\mathbf{1 1 d} \cdot \mathbf{2} \mathbf{H}_{2} \mathrm{O}$. IR (ATR, $\mathrm{cm}^{-1}$ ): 3332w, 3264m, 3237m, 3151m, 3068w, 2921m, $2874 \mathrm{~m}, 2851 \mathrm{w}, 2721 \mathrm{w}, 1632 \mathrm{~s}, 1619 \mathrm{~s}, 1594 \mathrm{~m}, 1562 \mathrm{~m}, 1507 \mathrm{~m}$, $1459 \mathrm{~m}, 1430 \mathrm{~m}, 1404 \mathrm{w}, 1359 \mathrm{~s}, 1342 \mathrm{~s}, 1302 \mathrm{w}, 1287 \mathrm{w}, 1261 \mathrm{~m}$, $1212 \mathrm{w}, 1177 \mathrm{~m}, 1167 \mathrm{~s}, 1149 \mathrm{~m}, 1113 \mathrm{w}, 1086 \mathrm{~s}, 1063 \mathrm{~s}, 1022 \mathrm{vs}$, 971w, 954w, 928s, 891m, 885s, $857 \mathrm{w}, 820 \mathrm{w}, 810 \mathrm{~m}, 779 \mathrm{vvs}, 742 \mathrm{~s}$, $703 \mathrm{~m}, 641 \mathrm{~s}, 632 \mathrm{~s}, 595 \mathrm{~s}, 553 \mathrm{~m}, 522 \mathrm{~s}, 503 \mathrm{~s}, 489 \mathrm{~s}, 438 \mathrm{~s}, 408 \mathrm{~m}$.

\section{Monitoring the conversion of anti-11d-4MeOH into syn-11d $2 \mathrm{H}_{2} \mathrm{O}$ with IR spectroscopy}

Few, freshly prepared crystals of anti-11d-4MeOH were placed in an ATR sample holder. At the start of the experiment, the spectra were collected every 5 minutes. The last spectrum was recorded after 24 hours. The spectra are shown in the ESI $\dagger$ (Fig. S20). The identity of the product was confirmed by PXRD (Fig. S1, ESI $\dagger$ ).

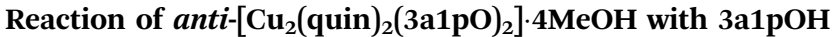

anti-11d.4MeOH $(50 \mathrm{mg})$ was stirred in acetonitrile $(7.5 \mathrm{~mL})$ with quinaldinic acid $(28 \mathrm{mg})$ and $3 \mathrm{a} 1 \mathrm{pOH}(33 \mathrm{mg})$ at room temperature for 2 days. Crystalline 11a was filtered off. Yield: $60 \mathrm{mg}$.

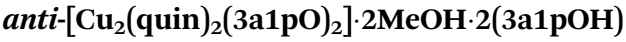 (anti-11d-2MeOH·2(3a1pOH))}

A Teflon container was filled with $\left[\mathrm{Cu}(\text { quin })_{2}\left(\mathrm{H}_{2} \mathrm{O}\right)\right](50 \mathrm{mg}$, $0.12 \mathrm{mmol})$, methanol $(7.5 \mathrm{~mL})$ and 3-amino-1-propanol $(150 \mathrm{mg})$. The container was closed and inserted into a steel autoclave, which was heated for 24 hours at $105{ }^{\circ} \mathrm{C}$. Afterwards, the autoclave was taken out from the oven and left overnight to cool to room temperature. The resulting green solution was concentrated under reduced pressure on a rotary evaporator. A glass vial with diethyl ether was carefully inserted into the Erlenmeyer flask with the concentrate. After a period of time, crystals of anti-11d-2MeOH.2(3a1pOH) grew from the concentrate. Please note that the crystals of anti-11d-2MeOH·2(3a1pOH) are not stable when taken out from the mother liquor. They were obtained only once. IR (ATR, $\mathrm{cm}^{-1}$ ): 3386w, 3341m, 3330m, 3271m, 3227m, 
$3152 \mathrm{w}, 3057 \mathrm{w}, 2927 \mathrm{~m}, 2902 \mathrm{w}, 2866 \mathrm{~m}, 2823 \mathrm{w}, 1628 \mathrm{vs}, 1593 \mathrm{~s}$, $1560 \mathrm{~s}, 1505 \mathrm{w}, 1459 \mathrm{~m}, 1431 \mathrm{~m}, 1365 \mathrm{vs}, 1343 \mathrm{~s}, 1304 \mathrm{w}, 1280 \mathrm{w}$, $1262 \mathrm{~m}, 1212 \mathrm{w}, 1167 \mathrm{~m}, 1147 \mathrm{~m}, 1113 \mathrm{w}, 1085 \mathrm{~m}, 1051 \mathrm{~s}, 995 \mathrm{~s}$, 983m, 958m, 933m, 899m, 893m, 885m, 855m, 810s, 803s, 793m, 774vvs, 738s, 705m, 640m, 630s, 619s, 597s, 560m, 520m, 495s, 458m, 436m.

\section{X-ray structure analysis}

Single crystal X-ray diffraction data were recorded using an Agilent SuperNova diffractometer with molybdenum (Mo-K $\lambda=0.71073 \AA)$ or copper $\left(\mathrm{Cu}-\mathrm{K}_{\alpha}, \lambda=1.54184 \AA\right)$ micro-focus sealed X-ray source at $150 \mathrm{~K}$. The diffractometer was equipped with mirror optics and an Atlas detector. Silicone grease was used to place each crystal on a fiber glass tip, which was then mounted on the goniometer head. Data were processed using CrysAlis PRO. ${ }^{40}$ Structures were solved with Olex $^{2}$ software ${ }^{41}$ using intrinsic phasing in ShelXT ${ }^{42}$ and refined with the least squares methods in ShelXL. ${ }^{43}$ Anisotropic displacement parameters were determined for all non-hydrogen atoms. Where possible, $\mathrm{NH}_{2}, \mathrm{NH}, \mathrm{NH}_{3}{ }^{+}$and $\mathrm{OH}$ hydrogen atoms of amino alcohols were located from a difference Fourier map and refined with isotropic displacement parameters. $\mathrm{OH}$ hydrogen atoms of water and methanol in anti-11d $4 \mathrm{MeOH}, \boldsymbol{s y n}-11 \mathrm{~d} \cdot 2 \mathrm{H}_{2} \mathrm{O}$ and anti-11d-2MeOH.2(3a1pOH) were located from a difference Fourier map. In some structures, the hydrogen atoms of amino alcohol heteroatoms were added in calculated positions due to ligand disorder (7a, 8c, 11b and 11c), large number of atoms in the asymmetric unit (8e and 10e) or a poor-quality data set (syn8d). The remaining hydrogen atoms were placed in geometrically calculated positions in all structures and refined using riding models. Disorder was observed for amino alcohol ligands in $\mathbf{2 b}$, $5 e, 7 a, 7 e, 8 c, 8 e, 10 e, 11 b$ and 11c. For $2 b$, the disorder over a twofold rotation axis pertains also to copper(II) ion. For all, the disorder was resolved using the appropriate PART instruction. The programs Platon, ${ }^{44}$ Ortep $^{45}$ and Mercury ${ }^{46}$ were used for crystal structure analysis and the preparation of figures. The crystallographic data are collected in Tables S1-S3 (ESI $\dagger$ ). All crystal structures were deposited at the CCDC and were assigned deposition numbers 2076993 (1a), 2076994 (2b), 2076995 (g), 2076996 (anti-4d), 2076997 (5e), 2076998 (6a), 2076999 (7a), 2077000 (7e), 2077001 (8b), 2077002 (8c), 2077003 (syn-8d), 2077004 (8e), 2077005 (9f), 2077006 (10e), 2077007 (11a), 2077008 (11a·3a1pOH), 2077009 (11b), 2077010 (11c), 2077011 (syn-11d), 2077012 (syn-11d·2 $\left.\mathbf{H}_{2} \mathbf{O}\right), 2077013$ (anti-11d·2MeCN), 2077014 (anti-11d·4MeOH), and 2077015 (anti-11d·2MeOH. $2(3 \mathrm{a} 1 \mathrm{pOH}))$.

\section{Computational details}

Geometries of copper(II) complexes were optimized without constraints from X-ray diffraction data, using the Gaussian 09 software. ${ }^{47}$ Coordinates of complex molecules of 11a, 11b, syn11d $\cdot 2 \mathrm{H}_{2} \mathrm{O}$ and $\boldsymbol{a n t i - 1 1 d} \cdot \mathbf{2 M e C N}$ were used. The initial geometry of 11a-L was derived from a repeating unit of 1D-polymer 11c. Vibrational analysis confirmed the nature of these structures as minima in the potential energy surface and was also used to obtain Zero Point and Gibbs free energies. The basis set $6-31+\mathrm{g}(\mathrm{d}, \mathrm{p})^{48,49}$ including diffusion and polarization was used for all atoms, except for $\mathrm{Cu}$, which was described by means of the SDD basis and the associated electron core potential (ECP).$^{50}$ The B3PW91 ${ }^{51-53}$ functional with the D3 version of Grimme's dispersion with Becke-Johnson damping ${ }^{54}$ was used. Bulk solvent effects (acetonitrile) were included during optimization with the SMD continuum model. ${ }^{55}$ Relative energies of the triplet and antiferromagnetically coupled singlet states were assessed for the syn and anti isomers of $\left[\mathrm{Cu}_{2} \text { (quin }\right)_{2}$ $\left.(3 \mathrm{a} 1 \mathrm{pO})_{2}\right]$ and the stabilities of the corresponding DFT wavefunctions were tested. ${ }^{56,57}$ Typically, RHF to UHF instabilities were found for wave functions obtained from spin-restricted (singlet) wavefunctions. When a more stable, spin-unrestricted, wavefunction was located, it was used as the initial guess for subsequent geometry optimization. Analysis of the electron density within the Atoms In Molecules (AIM) theory of R. F. W. Bader $^{58}$ was performed with the Multiwfn program, ${ }^{59,60}$ which was also used for Non-Covalent Interaction (NCI) Analysis. ${ }^{61}$ The Cylview visualization software ${ }^{62}$ was used to prepare some of the figures.

\section{Magnetic measurements}

All stable compounds, that could be obtained in pure form and in sufficient quantities, were subjected to magnetic susceptibility measurements using a MPMS-XL Quantum Design SQUID magnetometer which works between 1.8 and $400 \mathrm{~K}$ for dc applied fields ranging from -7 to $7 \mathrm{~T}$. Measurements were performed on analytically pure polycrystalline samples (25.91, 21.40, 22.34, 14.12, 19.13, 23.15, 20.09, 19.97, 20.34, 23.75, 18.15 and $18.30 \mathrm{mg}$ for $1 \mathrm{a}, \mathbf{2 b}$, anti-4d, 5e, 6a, 7a, 7e, 8e, 9f, 11a, syn-11d $\cdot 2 \mathrm{H}_{2} \mathrm{O}$, and $\mathrm{g}$, respectively) introduced in polyethylene bags $(3 \times 0.5 \times 0.02 \mathrm{~cm}$, weighing typically between 7 and $19 \mathrm{mg}$ ). Prior to the experiments, a $M v s$. $H$ measurement was performed at $100 \mathrm{~K}$ to confirm the absence of ferromagnetic impurities. Consistent dc susceptibility and in-phase ac susceptibility have been obtained between 1.85 and $15 \mathrm{~K}$. The data were corrected for the sample holder and the diamagnetic contribution of the sample.

\section{Results and discussion}

\subsection{Synthetic considerations}

In this study, a total of eleven different amino alcohols was reacted with the $\left[\mathrm{Cu}(\text { quin })_{2}\left(\mathrm{H}_{2} \mathrm{O}\right)\right]$ precursor. The studied systems yielded six types of compounds, labelled a to f, which contained both the quinaldinate and the amino alcohol ligand. A homoleptic quinaldinate complex $\left[\mathrm{Cu}(\text { quin })_{2}\right]_{n}$, labelled $\mathbf{g}$, was also obtained. All the novel products are listed in Scheme 4 . The product abbreviation consists of a number which represents the amino alcohol and a letter which represents the compound type.

Typically, a large excess of amino alcohol was used in the reaction. Such a ligand-to-metal ion ratio favors the formation of a complex with the maximum number of ligands. In our case, trans- $\left[\mathrm{Cu}(\text { quin })_{2} \mathrm{~L}_{2}\right]$, a type a complex with two amino 


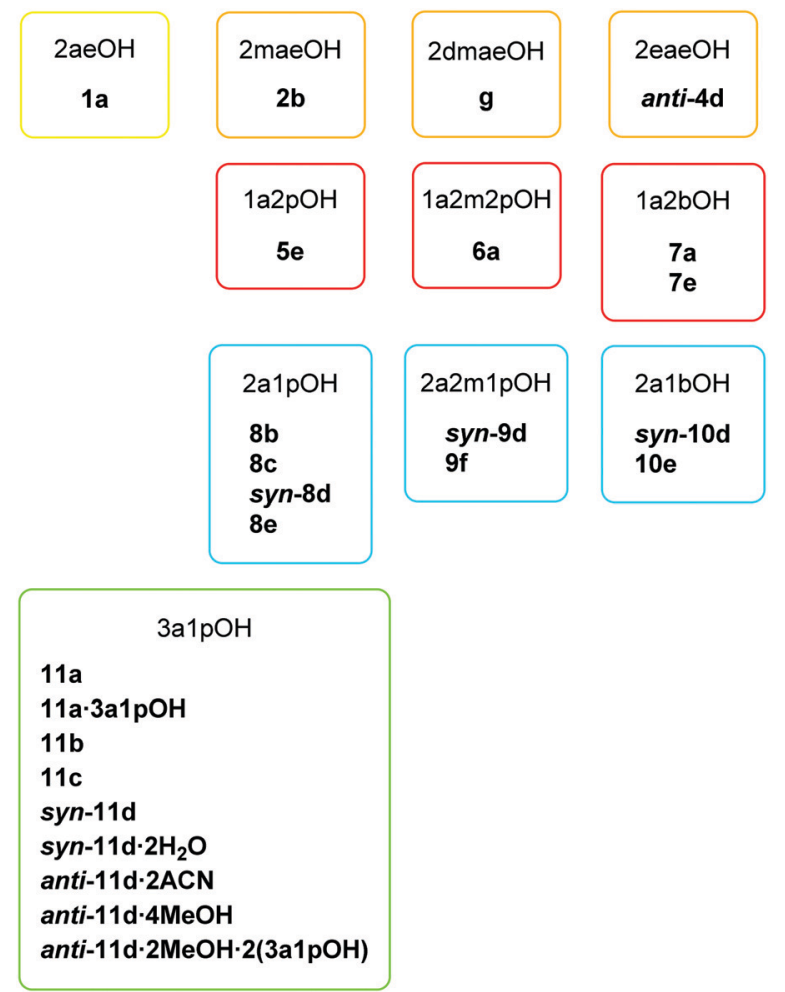

Scheme 4 List of novel copper(II) compounds with their abbreviation consisting of a number which represents the used amino alcohol and a letter which represents the compound type.

alcohol ligands L per metal ion, is obtained. Interestingly, only systems with 2-aminoethanol (1), 1-amino-2-methyl-2-propanol (6), 1-amino-2-butanol (7) and 3-amino-1-propanol (11) yielded such complexes. Our study shows that the amino alcohol ligands preferentially bind to copper(II) ions through their $\mathrm{NH}_{2}$ groups. The coordination behavior of the $N$-alkyl substituted amino alcohols, 2-methylaminoethanol (2), 2-dimethylaminoethanol (3) and 2-ethylaminoethanol (4), was thus expected to be drastically different. As mentioned below, the alkyl substituent on nitrogen neither prevents its coordination to copper(II) nor favors one coordination mode. For 2-dimethylaminoethanol (3) with two methyl substituents (the ligand with the greatest steric hindrance), no amino alcohol complex was isolated during the course of our work. Instead, a homoleptic quinaldinate complex, $\left[\mathrm{Cu}(\text { quin })_{2}\right]_{n}(\mathrm{~g})$ with an infinite chain structure, was obtained. A later rational synthesis of $\mathbf{g}$ ruled out 2-dimethylaminoethanol as a necessary reagent. It should be noted that many copper(II) complexes with 2-dimethylaminoethanol or its deprotonated form are known. ${ }^{29}$ In these com- reaction conditions. The 2-ethylaminoethanol (4) system also

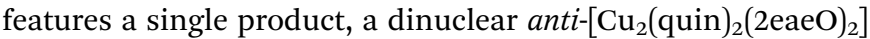
(anti-4d) with a deprotonated amino alcohol which is coordinated through its alkoxide oxygen and amino moiety. No auxiliary strong base was necessary to assist in the deprotonation of amino alcohols. Again, anti-4d formed both under mild reaction conditions, $4{ }^{\circ} \mathrm{C}$ or room temperature, and at $105{ }^{\circ} \mathrm{C}$ in an autoclave.

A key information obtained from these eleven systems is that the nature of the product depends more upon the amino alcohol used than the applied reaction conditions. For example, 2-aminoethanol (1), 1-amino-2-propanol (5) and 1-amino-2methyl-2-propanol (6), yielded in combination with the $\left.[\mathrm{Cu} \text { (quin })_{2}\left(\mathrm{H}_{2} \mathrm{O}\right)\right]$ starting material a single product, both under mild and under forcing reaction conditions. The amino alcohols 1 and 6 afforded type a complexes, [Cu(quin $\left.)_{2}(2 \mathrm{aeOH})_{2}\right]$ (1a) and $\left[\mathrm{Cu}(\text { quin })_{2}(1 \mathrm{a} 2 \mathrm{~m} 2 \mathrm{pOH})_{2}\right](6 \mathbf{6})$, whereas for 5 , an ionic $\left[\mathrm{Cu}(1 \mathrm{a} 2 \mathrm{pOH})_{3}\right](\text { quin })_{2}$ (5e) was obtained. The formation of a homoleptic amino alcohol complex from [Cu(quin $\left.)_{2}\left(\mathrm{H}_{2} \mathrm{O}\right)\right]$ under mild conditions is surprising. The $\left\{\mathrm{Cu}(\text { quin })_{2}\right\}$ fragment was not expected to fall apart. Instead, in the presence of 1-amino-2-propanol (5), the copper(II) coordination sphere was almost instantaneously depleted of bidentate chelating quinaldinates. Each of the systems with 1-amino-2-butanol (7), 2-amino-1-propanol (8), 2-amino-2-methyl-1-propanol (9), 2-amino-1-butanol (10) and 3-amino-1-propanol (11) has led to more than one product. Reactions with 2-amino-2-methyl-1propanol (9) afforded a dinuclear type d complex with a syn disposition of aromatic ligands, $\operatorname{syn}-\left[\mathrm{Cu}_{2}(\text { quin })_{2}(2 \mathrm{a} 2 \mathrm{~m} 1 \mathrm{pO})_{2}\right]$ (syn-9d), and $\left[\mathrm{Cu}(2 \mathrm{a} 2 \mathrm{~m} 1 \mathrm{pO})_{2}\right](2 \mathrm{a} 2 \mathrm{~m} 1 \mathrm{pOHH})_{2}(\text { quin })_{2}$ (9f), a co-crystal which contains a homoleptic amino alcoholate complex. The 2-amino-2-methyl-1-propanol system is the only one that produced the type $\mathbf{f}$ complex. Dinuclear complex syn9d co-crystallized with the amino alcohol salt with quinaldinate, $2 \mathrm{a} 2 \mathrm{~m} 1 \mathrm{pOHH}^{+}$quin $^{-}\left(2 \mathrm{a} 2 \mathrm{~m} 1 \mathrm{pOHH}^{+}=\right.$a cationic form of amino alcohol with a $\mathrm{NH}_{3}{ }^{+}$moiety). If the latter mixture was left undisturbed for a couple of days, both solids were consumed and a new phase, purple crystals of $\left[\mathrm{Cu}(2 \mathrm{a} 2 \mathrm{~m} 1 \mathrm{pO})_{2}\right](2 \mathrm{a} 2 \mathrm{~m} 1-$ pOHH $)_{2}(\text { quin })_{2}(\mathbf{9 f})$ appeared. This sequence of events suggests the origin of $2 \mathrm{a} 2 \mathrm{~m} 1 \mathrm{pOHH}^{+}$and $2 \mathrm{a} 2 \mathrm{~m}_{1} \mathrm{pO}^{-}$ions. The following reaction implies a transfer of the $\mathrm{OH}$ proton from one amino alcohol molecule to the $\mathrm{NH}_{2}$ group in another:

$$
2 \mathrm{a} 2 \mathrm{~m} 1 \mathrm{pOH}+2 \mathrm{a} 2 \mathrm{~m} 1 \mathrm{pOH} \rightleftharpoons 2 \mathrm{a} 2 \mathrm{~m} 1 \mathrm{pOHH}^{+}+2 \mathrm{a} 2 \mathrm{~m} 1 \mathrm{pO}^{-}
$$

The coordination of thus formed $2 \mathrm{a} 2 \mathrm{~m} 1 \mathrm{pO}^{-}$ions to copper(II) could be the driving force for this reaction. Once the amount of the amino alcoholate ions is large enough, a successive reaction, conversion of syn-9d into $\mathbf{9 f}$, can take place:

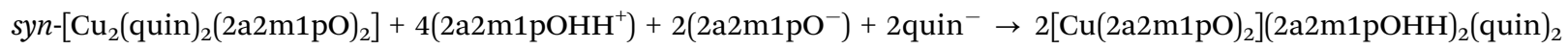

plexes, 2dmaeOH adopts a bidentate chelating coordination. The ligand with one methyl group, 2-methylaminoethanol (2), coordinates to copper(II) in a bidentate chelating manner. The type b complex, $\left[\mathrm{Cu}(\text { quin })_{2}(2 \mathrm{maeOH})\right](\mathbf{2 b})$, is the only species that could be isolated from this ligand system regardless of the
The 2-amino-1-butanol (10) system is another example with the confirmed formation of the $2 \mathrm{a} 1 \mathrm{bOHH}^{+}$quin ${ }^{-}$salt. Similar to the 2-amino-2-methyl-1-propanol (9) ligand system, the salt and the dinuclear complex with amino alcoholate ligands, syn-10d, crystallized simultaneously. 
The ligand systems of 2-amino-1-propanol (8) with four products and 3-amino-1-propanol (11) with nine products displayed the greatest structural diversity among the isolated products. The reactions with $\mathbf{8}$ turned out to be very erratic. Despite numerous and methodical attempts, no reproducible synthetic conditions necessary for the formation of pure $\left[\mathrm{Cu}(\text { quin })_{2}(2 \mathrm{a} 1 \mathrm{pOH})\right] \quad(\mathbf{8 b}), \quad\left[\mathrm{Cu}(\text { quin })_{2}(2 \mathrm{a} 1 \mathrm{pOH})\right]_{n} \quad(\mathbf{8 c})$, syn$\left.\left[\mathrm{Cu}_{2} \text { (quin }\right)_{2}(2 \mathrm{a} 1 \mathrm{pO})_{2}\right](\boldsymbol{s y n - 8 d})$ or $\left[\mathrm{Cu}(2 \mathrm{a} 1 \mathrm{pOH})_{3}\right](\text { quin })_{2} \quad(\mathbf{8 e})$ could be established. The poor reproducibility of these reactions remains our most worrisome concern. Conversely, the 3-amino-1propanol ligand system displays more control over the reaction outcome. The type a complex, $\left[\mathrm{Cu}(\text { quin })_{2}(3 \mathrm{a} 1 \mathrm{pOH})_{2}\right]$ (11a), appears as the thermodynamic product. Several $3 \mathrm{a} 1 \mathrm{pOH}$ and $3 \mathrm{a} \mathrm{pO}^{-}$compounds were shown to transform into 11a. The type a complex crystallizes also as a solvate, $\left[\mathrm{Cu}(\text { quin })_{2}(3 \mathrm{a} 1 \mathrm{pOH})_{2}\right]$. $3 \mathrm{a} 1 \mathrm{pOH}(\mathbf{1 1 a} \cdot 3 \mathrm{a} 1 \mathrm{pOH})$. The crystals of 11a.3a1pOH are not stable, neither in air nor in solution. In both cases, they transform into 11a. Interestingly, the $3 \mathrm{a} 1 \mathrm{pOH}$ system yielded two mono amino alcohol compounds with the $\left[\mathrm{Cu}(\text { quin })_{2}(3 \mathrm{a} 1 \mathrm{pOH})\right]$ composition, a mononuclear 11b and a 1D-polymer 11c. Mononuclear 11b features a bidentate chelating amino alcohol with the hydroxylto-copper(II) bond distance being on the verge of the acceptable bonding interactions. 11b was obtained only once, otherwise a complex with two amino alcohol ligands per copper(II) ion formed. The reaction of $\mathbf{1 1} \mathbf{b}$ with the excess ligand confirms its metastable nature. In 1D-polymer 11c, the amino alcohol ligand is engaged in coordination both functional groups, yet to two metal ions. The composition of the reaction mixture that

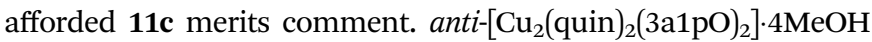
(anti-11d-4MeOH) with a dinuclear core reacted with quinaldinic acid to form 11c in the mixture of 3-amino-1-propanol, acetonitrile and methanol. The rigidity of the dinuclear core in anti-11d.4MeOH was ruled out also by its conversion into $11 \mathrm{a}$ and its reaction with the air moisture to syn$\left[\mathrm{Cu}_{2}(\text { quin })_{2}(3 \mathrm{a} 1 \mathrm{pO})_{2}\right] \cdot 2 \mathrm{H}_{2} \mathrm{O}\left(\boldsymbol{s y n}-\mathbf{1 1 d} \cdot \mathbf{2} \mathbf{H}_{2} \mathrm{O}\right)$. The latter reaction is not surprising, as $\boldsymbol{s y n - 1 1 d} \cdot \mathbf{2} \mathbf{H}_{2} \mathrm{O}$ usually crystallized from reaction mixtures that contained water which was introduced unintentionally, for example, with triethylamine. The latter was added with the aim to assist in the deprotonation of amino alcohol. What makes the conversion of anti-11d-4MeOH into syn-11d $2 \mathbf{H}_{2} \mathrm{O}$ interesting is the isomerization that also takes place. The conversion was monitored by IR vibrational spectroscopy, whereas the identity of the final product was confirmed by PXRD (ESI, $\dagger$ Fig. S1). The $3 \mathrm{a} 1 \mathrm{pOH}$ reaction system afforded

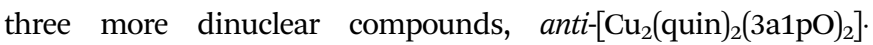
$2 \mathrm{MeCN}$ (anti-11d·2MeCN), syn- $\left[\mathrm{Cu}_{2}(\text { quin })_{2}(3 \mathrm{a} 1 \mathrm{pO})_{2}\right]$ (syn-11d) and

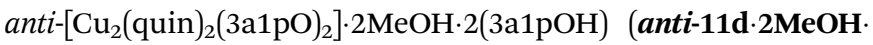
2(3a1pOH)). Their crystallization depends critically upon the content of solvents. The difficulties to control it, explain why, for example, the methanol/3-amino-1-propanol solvate, anti-11d. 2МеOH·2(3a1pOH), was obtained only once. The closing remark pertains to the syn-/anti-isomerism observed for a dinuclear $3 \mathrm{a} 1 \mathrm{pO}^{-}$complex. Since both isomers can crystallize from the same reaction mixture, the reaction conditions do not favor one isomer over another.

\subsection{Description of crystal structures}

The descriptions are ordered according to the structural types shown in Scheme 3. Most relevant geometric parameters are given in Tables 1-4. trans-[Cu(quin $\left.)_{2}(2 \mathrm{aeOH})_{2}\right](\mathbf{1 a})$, trans-[Cu(quin $)_{2}$ $\left.(1 \mathrm{a} 2 \mathrm{~m} 2 \mathrm{pOH})_{2}\right](6 \mathbf{a})$, trans-[Cu(quin $\left.)_{2}(1 \mathrm{a} 2 \mathrm{bOH})_{2}\right]$ (7a), trans-[Cu(quin $\left.)_{2}(3 \mathrm{a} 1 \mathrm{pOH})_{2}\right]\left(\right.$ 11a) and trans $-\left[\mathrm{Cu}(\text { quin })_{2}(3 \mathrm{a} 1 \mathrm{pOH})_{2}\right] \cdot 3 \mathrm{a} 1 \mathrm{pOH}$

Table 1 Relevant geometric parameters $\left[\AA \AA{ }^{\circ}\right]$ for complexes with monodentate amino alcohol ligands

\begin{tabular}{llllll}
\hline Compound & Amino alcohol & $\mathrm{Cu}-\mathrm{NH}_{2}$ & $\mathrm{Cu}-\mathrm{N}\left(\right.$ quin $\left.^{-}\right)$ & $\mathrm{Cu}-\mathrm{O}\left(\right.$ quin $\left.^{-}\right)$ & Non-planarity of quin $^{-a}$ \\
\hline 1a & 2aeOH & $2.0014(15)$ & $2.3498(14)$ & $2.0813(12)$ & $4.6(3)$ \\
6a & 1a2m2pOH & $2.0193(14)$ & $2.3916(14)$ & $2.0445(12)$ & $5.3(3)$ \\
7a & 1a2bOH & $2.0033(15)$ & $2.4130(16)$ & $2.0355(12)$ & $2.5(3)$ \\
11a & 3a1pOH & $2.0184(14)$ & $2.4185(14)$ & $2.0034(11)$ & $10.3(2)$ \\
11a·3a1pOH & 3a1pOH & $2.0177(16), 2.0263(15)$ & $2.3627(15), 2.3869(15)$ & $2.0149(12), 2.0168(12)$ & $4.22(12), 6.3(3)$
\end{tabular}

${ }^{a}$ Given by the dihedral angle between the carboxylate and the bicyclic system.

Table 2 Relevant bond lengths [Å] for complexes with bidentate chelating amino alcohol ligands

\begin{tabular}{|c|c|c|c|c|c|}
\hline Compound & $\begin{array}{l}\text { Amino } \\
\text { alcohol }\end{array}$ & $\mathrm{Cu}$-amino group & $\mathrm{Cu}-\mathrm{OH}$ & $\mathrm{Cu}-\mathrm{N}\left(\right.$ quin $\left.^{-}\right)$ & $\mathrm{Cu}-\mathrm{O}\left(\right.$ quin $\left.^{-}\right)$ \\
\hline $2 \mathbf{b}$ & $2 \mathrm{maeOH}$ & $2.052(2)$ & $2.517(2)$ & $2.0353(13)$ & $1.9379(15)$ \\
\hline $11 b$ & 3a1pOH & $1.953(4)$ & $2.510(4)$ & $2.2408(13)$ & $1.9468(11)$ \\
\hline $5 e$ & $1 \mathrm{a} 2 \mathrm{pOH}$ & $1.998(2), 1.999(2), 2.057(2)$ & $2.0130(19), 2.3862(19), 2.4029(19)$ & - & - \\
\hline $7 e$ & $1 \mathrm{a} 2 \mathrm{bOH}$ & $1.9945(19), 1.9982(19), 2.035(2)$ & $2.0191(19), 2.3824(18), 2.4558(17)$ & - & - \\
\hline $10 e$ & $2 \mathrm{a} 1 \mathrm{bOH}$ & $\begin{array}{l}1.991(3), 1.997(3), 1.998(3), 2.000(3), \\
2.004(4), 2.004(3), 2.009(3), 2.010(3) \\
2.011(3), 2.019(3), 2.019(3), 2.020(3)\end{array}$ & $\begin{array}{l}1.995(3), 1.997(3), 2.005(3), 2.015(3), \\
2.331(3), 2.355(3), 2.381(3), 2.389(3), \\
2.462(3), 2.526(3), 2.550(3), 2.609(3)\end{array}$ & - & - \\
\hline
\end{tabular}


Table 3 Relevant bond lengths [Å] for complexes with bidentate bridging amino alcohol ligands

\begin{tabular}{llllll}
\hline \multicolumn{1}{c}{$\begin{array}{l}\text { Amino } \\
\text { Compound } \\
\text { alcohol }\end{array}$} & ${\mathrm{Cu}-\mathrm{NH}_{2}}$ & $\mathrm{Cu}-\mathrm{OH}$ & $\mathrm{Cu}-\mathrm{N}\left(\right.$ quin $\left.^{-}\right)$ & $\mathrm{Cu}-\mathrm{O}\left(\right.$ quin $\left.^{-}\right)$ \\
\hline 8c & $2 \mathrm{a} 1 \mathrm{pOH}$ & $2.056(5)$ & $2.288(4)$ & $2.2466(19)$ & $1.9535(14)$ \\
11c & $3 \mathrm{a} 1 \mathrm{pOH}$ & $2.079(6)$ & $2.286(5)$ & $2.2447(17)$ & $1.9460(12)$
\end{tabular}

(11a-3a1pOH) belong to type a complexes which have in common that copper(II) is coordinated by two bidentate chelating quinaldinates and two monodentate amino alcohol ligands bound via $\mathrm{N}$-donor atom of the amino moiety. The ORTEP drawing of 11a is shown in Fig. 1. The arrangement of ligands is trans. All type a complexes are centrosymmetric with the $\mathrm{N}_{4} \mathrm{O}_{2}$ donor set defining vertices of a distorted octahedron. Due to Jahn-Teller effect which is a common phenomenon for $\mathrm{d}^{9}$ ions, ${ }^{63}$ the octahedron is elongated. This bonding pattern is usually described as a " $4+2$ ", coordination. ${ }^{6}$ The two longer bonds are observed to the quinaldinate nitrogen atoms. The aromatic ligands deviate from strict planarity. Their non-planarity can be described by a dihedral angle between the carboxylate and the bicyclic system. The deviation is most pronounced for 11a with an angle of 10.3(2) .

$\left[\mathrm{Cu}(\text { quin })_{2}(2 \mathrm{maeOH})\right]$ (2b), $\left[\mathrm{Cu}(\text { quin })_{2}(2 \mathrm{a} 1 \mathrm{pOH})\right](\mathbf{8 b})$ and $\left[\mathrm{Cu}(\text { quin })_{2}(3 \mathrm{a} 1 \mathrm{pOH})\right]$ (11b) fall under type b compounds. In all, copper(II) ion is coordinated by two bidentate chelating quinaldinates and a bidentate chelating amino alcohol bound via both functional groups. Thus, the metal ion acquires the $\mathrm{N}_{3} \mathrm{O}_{3}$ coordination environment. The ORTEP drawing of [Cu(quin) $)_{2}^{-}$ (2a1pOH)] (8b) is shown in Fig. 2. Complex molecules of $\mathbf{2 b}$ and 11b possess a crystallographically imposed $C_{2}$ symmetry. Hence, their amino alcohol ligands exhibit disorder. In spite of the long bond between the metal ion and the $\mathrm{OH}$ moiety,

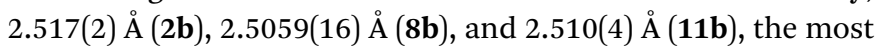
appropriate description of the amino alcohol binding mode remains a bidentate chelating one.

$\left[\mathrm{Cu}(\text { quin })_{2}(2 \mathrm{a} 1 \mathrm{pOH})\right]_{n}$ (8c) and $\left[\mathrm{Cu}(\text { quin })_{2}(3 \mathrm{a} 1 \mathrm{pOH})\right]_{n}$ (11c) belong to type complexes. The coordination environment of copper(II) consists of two bidentate chelating quinaldinates and two amino alcohol ligands, one being coordinated via $\mathrm{NH}_{2}$ and the other via $\mathrm{OH}$ moiety. The distribution of the $\mathrm{N}_{3} \mathrm{O}_{3}$ donors describes a distorted octahedron. The binding mode of the amino alcohol ligand is bidentate bridging, i.e., the amino moiety is bound to one metal ion and the hydroxyl moiety to a neighboring one. As a result, an infinite chain structure is formed. The ORTEP drawings of a repeating unit and a short

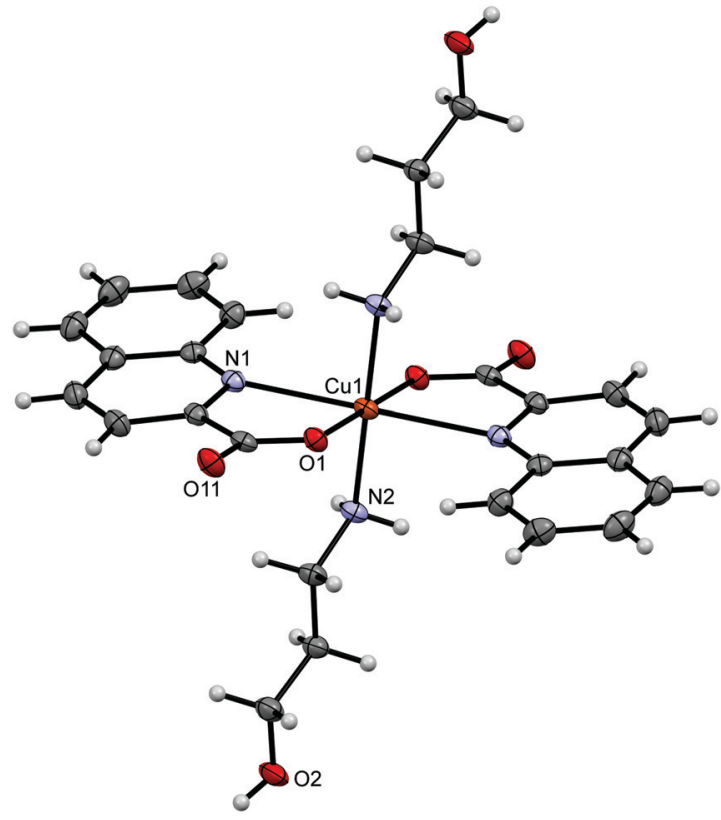

Fig. 1 ORTEP drawing of trans-[Cu(quin $\left.)_{2}(3 a 1 p O H)_{2}\right]$ (11a). Displacement ellipsoids are drawn at the $50 \%$ probability level. Hydrogen atoms are shown as spheres of arbitrary radii.

section of a chain in $\left[\mathrm{Cu}(\text { quin })_{2}(2 \mathrm{a} 1 \mathrm{pOH})\right]_{n}(\mathbf{8 c})$ are shown in Fig. 3.

Type d represents dinuclear complexes in which two copper(II) ions are bridged by two oxygen atoms, each belonging to a deprotonated amino alcohol. The resulting intermetallic distance is in the 2.9177(9)-3.0541(4) A range. Both copper(II) ions share the same coordination environment which consists of a bidentate chelating quinaldinate and two amino alcoholate ions. As usually observed, the amino alcoholate engages in coordinating both donor groups: ${ }^{29}$ the amino group is bound to one metal ion, while the alkoxide oxygen is bound to two of them. In Harris notation, this binding mode is labelled 2.21. ${ }^{64}$ The metal ion is five-coordinated by $\mathrm{N}_{2} \mathrm{O}_{3}$ donors. With $\tau$ parameters in the 0.02 to 0.33 range, the geometry can be best described as a square pyramid. ${ }^{65}$ The dinuclear complexes exist in two isomeric forms which differ in the relative arrangement of quinaldinates with respect to the $\mathrm{Cu}_{2}(\mu-\mathrm{O})_{2}$ plane. In syn isomers, the quinaldinates are on the same side of this plane. Such an arrangement allows intramolecular $\pi \cdots \pi$ stacking (Table S8, ESI $\dagger$ ). Complex molecules of $s y n-\left[\mathrm{Cu}_{2}(\text { quin })_{2}(3 \mathrm{a} 1 \mathrm{pO})_{2}\right]$ (syn-11d) and $\left.s y n-\left[\mathrm{Cu}_{2} \text { (quin }\right)_{2}(3 \mathrm{a} 1 \mathrm{pO})_{2}\right] \cdot 2 \mathrm{H}_{2} \mathrm{O} \quad\left(\boldsymbol{s y n}-\mathbf{1 1 d} \cdot 2 \mathbf{H}_{2} \mathbf{O}\right)$ have no

Table 4 Relevant geometric parameters [Å] for complexes with amino alcoholate ligands

\begin{tabular}{|c|c|c|c|c|c|c|}
\hline Compound & Amino alcohol & $\mathrm{Cu}$-amino group & $\mathrm{Cu}-\mathrm{O}^{-}$ & $\mathrm{Cu}-\mathrm{N}\left(\right.$ quin $\left.^{-}\right)$ & $\mathrm{Cu}-\mathrm{O}\left(\right.$ quin $\left.^{-}\right)$ & $\tau^{65}$ \\
\hline syn-8d & 2a1pOH & $2.001(3)$ & $1.941(3), 1.974(3)$ & $2.225(3)$ & $1.967(3)$ & 0.31 \\
\hline syn-11d & $3 \mathrm{a} 1 \mathrm{pOH}$ & $1.984(2), 2.004(2)$ & $1.9174(15)-1.9626(15)$ & $2.3088(17), 2.308(2)$ & $1.9728(15), 1.9695(16)$ & $0.13,0.20$ \\
\hline$s y n-11 d \cdot 2 \mathrm{H}_{2} \mathrm{O}$ & $3 \mathrm{a} 1 \mathrm{pOH}$ & $1.981(2), 1.980(2)$ & $1.9275(16)-1.9432(17)$ & $2.310(2), 2.3170(19)$ & $2.0041(17), 2.0012(17)$ & $0.02,0.13$ \\
\hline anti-4d & $2 \mathrm{eaeOH}$ & $2.039(3)$ & $1.929(2), 1.945(2)$ & $2.281(2)$ & $1.962(2)$ & 0.33 \\
\hline anti-11d·2MeCN & 3a1pOH & $1.9946(14)$ & $1.9270(12), 1.9459(12)$ & $2.0472(14)$ & $2.2271(13)$ & 0.05 \\
\hline anti-11d.4MeOH & $3 a 1 \mathrm{pOH}$ & $2.0007(13)$ & $1.9211(10), 1.9581(10)$ & $2.0189(12)$ & $2.2248(11)$ & 0.12 \\
\hline anti-11d·2MeOH·2(3a1pOH) & $3 \mathrm{a} 1 \mathrm{pOH}$ & $1.9921(13)$ & $1.9353(10), 1.9518(10)$ & $2.0422(12)$ & $2.2311(11)$ & 0.09 \\
\hline 9f & $2 \mathrm{a} 2 \mathrm{~m} 1 \mathrm{pOH}$ & $1.9935(13)$ & $1.9162(10)$ & - & - & - \\
\hline
\end{tabular}




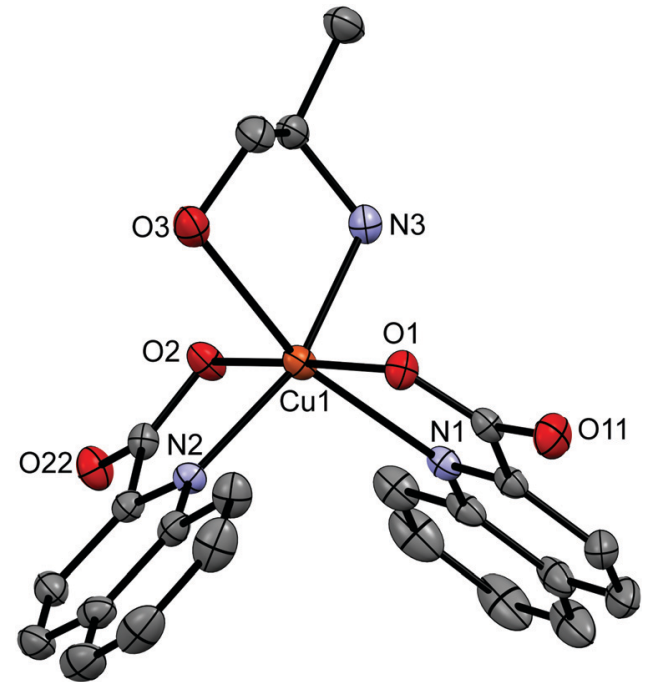

Fig. 2 ORTEP drawing of [Cu(quin) $\left.)_{2}(2 \mathrm{a} 1 \mathrm{pOH})\right]$ (8b). Displacement ellipsoids are drawn at the $50 \%$ probability level. Hydrogen atoms are omitted for clarity.

symmetry, while syn- $\left[\mathrm{Cu}_{2}(\text { quin })_{2}(2 \mathrm{a} 1 \mathrm{pO})_{2}\right]($ syn-8d) belongs to the $C_{2}$ point group symmetry. Interestingly, syn-8d is the only dinuclear complex that features a non-planar $\mathrm{Cu}_{2}(\mu-\mathrm{O})_{2}$ unit with the fold angle between its $\mathrm{Cu}(\mu-\mathrm{O})_{2}$ planes being $20.64(8)^{\circ}$. Fig. 4 shows the ORTEP drawing of syn-11d. The relative distribution of the $\mathrm{N}_{2} \mathrm{O}_{3}$ donors in syn isomers is the same: the quinaldinate oxygen occupies an equatorial site and the quinaldinate nitrogen the apical one. On the other hand, anti isomers have their quinaldinates located on the opposite sides of the $\mathrm{Cu}_{2}(\mu-\mathrm{O})_{2}$ plane, i.e., one quinaldinate is above and the other one below this plane. With such an arrangement of the aromatic ligands, intramolecular $\pi \cdots \pi$ interactions are not possible. The complex molecules of anti-[ $\left.\mathrm{Cu}_{2}(\text { quin })_{2}(2 \mathrm{eaeO})_{2}\right]$ (anti-4d), anti$\left[\mathrm{Cu}_{2}(\text { quin })_{2}(3 \mathrm{a} 1 \mathrm{pO})_{2}\right] \cdot 2 \mathrm{MeCN}$ (anti-11d-2MeCN), anti-[ $\left[\mathrm{Cu}_{2}(\text { quin })_{2}-\right.$ $\left.(3 \mathrm{a} 1 \mathrm{pO})_{2}\right] \cdot 4 \mathrm{MeOH}$ (anti-11d-4MeOH) and anti-[ $\mathrm{Cu}_{2}$ (quin) $)_{2}-$ $\left.(3 \mathrm{a} 1 \mathrm{pO})_{2}\right] \cdot 2 \mathrm{MeOH} \cdot 2(3 \mathrm{a} 1 \mathrm{pOH}) \quad($ anti-11d-2MeOH$\cdot 2(3 \mathrm{a} 1 \mathrm{pOH}))$ are centrosymmetric with a planar $\mathrm{Cu}_{2}(\mu-\mathrm{O})_{2}$ unit. Fig. 5 shows

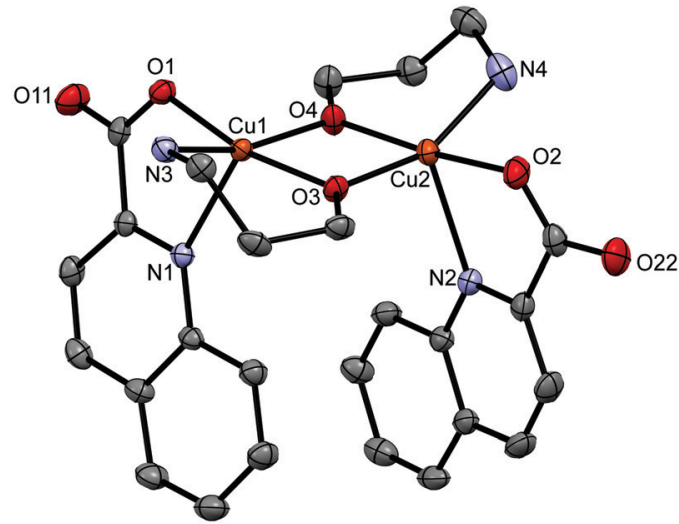

Fig. 4 ORTEP drawing of syn- $\left.\left[\mathrm{Cu}_{2} \text { (quin }\right)_{2}(3 \mathrm{a} 1 \mathrm{pO})_{2}\right]$ (syn-11d). Displacement ellipsoids are drawn at the $50 \%$ probability level. Hydrogen atoms are omitted for clarity.

the ORTEP drawings of complex molecules in anti-4d and anti11d-2MeCN. It is to be noted that the relative distribution of the $\mathrm{N}_{2} \mathrm{O}_{3}$ donors in anti-4d differs from the one in other anti compounds. In anti-4d, the quinaldinate oxygen occupies an equatorial site and the quinaldinate nitrogen the apical one, whereas all other compounds feature a reversed arrangement.

The ionic compounds, $\left[\mathrm{Cu}(1 \mathrm{a} 2 \mathrm{pOH})_{3}\right](\text { quin })_{2}(\mathbf{5 e}),[\mathrm{Cu}(1 \mathrm{a} 2 \mathrm{~b}-$ $\left.\mathrm{OH})_{3}\right](\text { quin })_{2}(7 \mathbf{e}),\left[\mathrm{Cu}(2 \mathrm{a} 1 \mathrm{pOH})_{3}\right](\text { quin })_{2}(8 \mathrm{e})$ and $\left[\mathrm{Cu}(2 \mathrm{a} 1 \mathrm{bOH})_{3}\right]-$ (quin) $)_{2}(\mathbf{1 0 e})$, feature a positively charged copper(II) complex with three amino alcohol ligands coordinated in a bidentate chelating manner and two quinaldinate ions as counter anions. The ORTEP drawing of the $\left[\mathrm{Cu}(1 \mathrm{a} 2 \mathrm{bOH})_{3}\right]^{2+}$ ion in $7 \mathrm{e}$ is shown in Fig. 6. The $\mathrm{N}_{3} \mathrm{O}_{3}$ donor set occupies vertices of a distorted octahedron in a mer distribution. The bonding pattern matches the " $4+2$ " description with the two longer bonds formed between copper(II) and the hydroxyl oxygen.

A co-crystal $\left[\mathrm{Cu}(2 \mathrm{a} 2 \mathrm{~m} 1 \mathrm{pO})_{2}\right](2 \mathrm{a} 2 \mathrm{~m} 1 \mathrm{pOHH})_{2}$ (quin $)_{2}$ (9f) consists of a neutral complex $\left[\mathrm{Cu}(2 \mathrm{a} 2 \mathrm{~m} 1 \mathrm{pO})_{2}\right]$ with two amino alcoholate ligands coordinated in a bidentate chelating manner (Fig. 7), $\mathrm{NH}_{2}$-protonated amino alcohol species and quinaldinate anions in the $1: 2: 2$ ratio. The four-coordinate
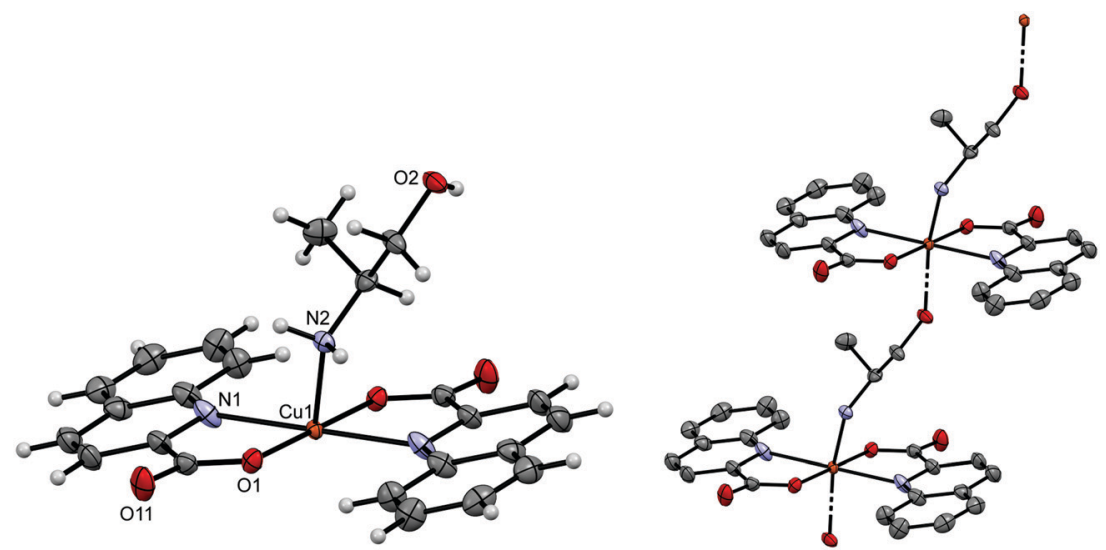

Fig. 3 ORTEP drawings of a repeating unit (left) and a short section of a chain (right) in [Cu(quin) $\left.{ }_{2}(2 \mathrm{a} 1 \mathrm{pOH})\right]_{n}(\mathbf{8 c})$. The $\mathrm{Cu}(\|)$ coordination sphere is completed by the O1, N1 and N2/O2 $(1-x, 1-y, 1-z)$ symmetry equivalents. Displacement ellipsoids are drawn at the $50 \%$ probability level. Hydrogen atoms are shown as spheres of arbitrary radii (left) or omitted for clarity (right). 

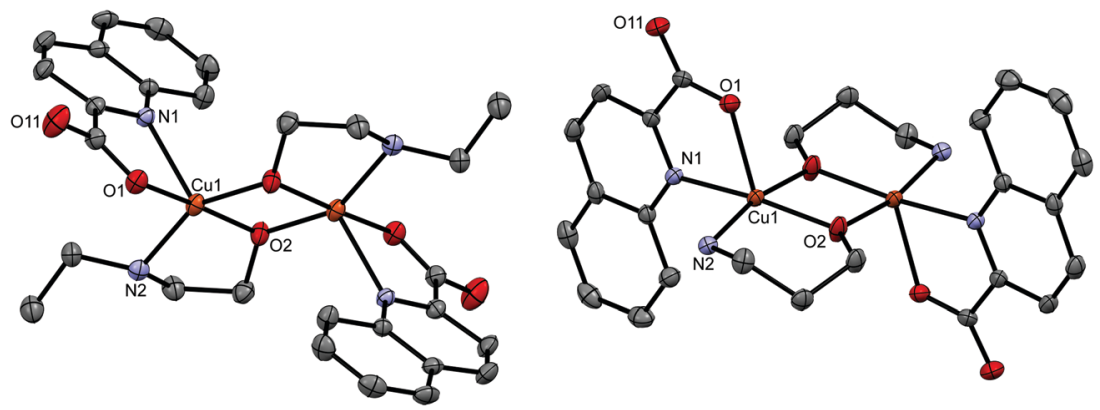

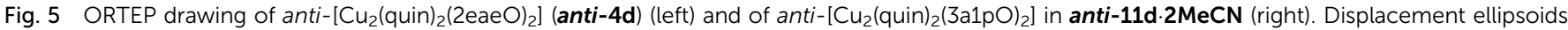
are drawn at the $50 \%$ probability level. Hydrogen atoms are omitted for clarity.

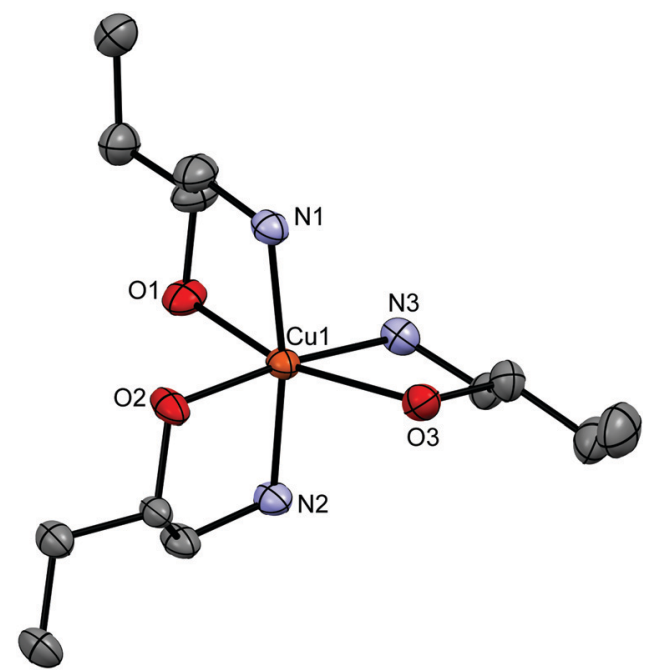

Fig. 6 ORTEP drawing of $\left[\mathrm{Cu}(1 \mathrm{a} 2 \mathrm{bOH})_{3}\right]^{2+}$ in 7e. Displacement ellipsoids are drawn at the $50 \%$ probability level. Hydrogen atoms are omitted for clarity.

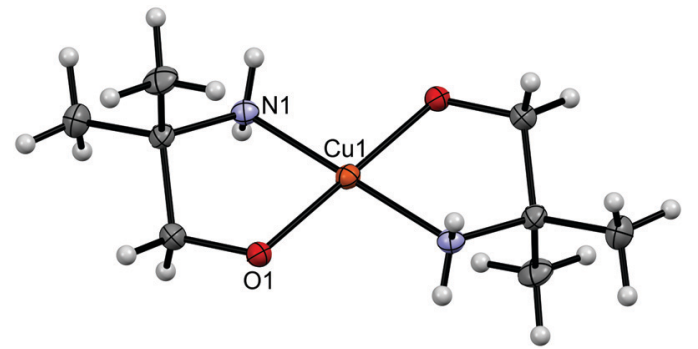

Fig. 7 ORTEP drawing of $\left[\mathrm{Cu}(2 \mathrm{a} 2 \mathrm{~m} 1 \mathrm{pO})_{2}\right]$ in 9 f. Displacement ellipsoids are drawn at the $50 \%$ probability level. Hydrogen atoms are shown as spheres of arbitrary radii.

$\left[\mathrm{Cu}(2 \mathrm{a} 2 \mathrm{~m} 1 \mathrm{pO})_{2}\right]$ is centrosymmetric with the $\mathrm{N}_{2} \mathrm{O}_{2}$ donors in a square-planar arrangement. A set of the closest neighbors of copper(II) ion consists apart from the $\mathrm{N}_{2} \mathrm{O}_{2}$ donors also of two hydroxyl groups of the $2 \mathrm{a} 2 \mathrm{~m} 1 \mathrm{pOHH}^{+}$cations at a distance of 3.5197(12) A.

$\left[\mathrm{Cu}(\text { quin })_{2}\right]_{n}$ (g) is a one-dimensional coordination polymer which may be regarded as an infinite repetition of mononuclear $\left[\mathrm{Cu}(\text { quin })_{2}\right]$ units. These mononuclear units are linked

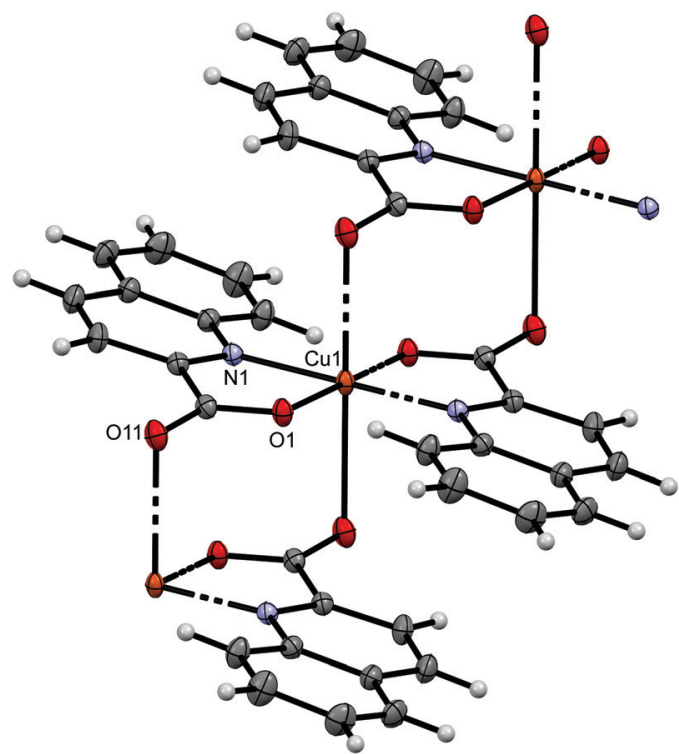

Fig. 8 ORTEP drawing of a short section of a chain in $\left[\mathrm{Cu}(\mathrm{quin})_{2}\right]_{n}(\mathbf{g})$. Displacement ellipsoids are drawn at the $50 \%$ probability level. Hydrogen atoms are shown as spheres of arbitrary radii. The $\mathrm{Cu}(\|)$ coordination sphere is completed by the $\mathrm{O} 11(-x, 1-y, 1-z$ and $1+x, y, z), \mathrm{N} 1$ and $\mathrm{O} 1$ $(1-x, 1-y, 1-z)$ symmetry equivalents. The $\mathrm{Cu}-\mathrm{N}$ bond lengths are 2.0907(12) $\AA$, and the $\mathrm{Cu}-\mathrm{O}$ bond lengths are 1.9089(10) and 2.5772(12) $\AA$.

with the agency of long $\mathrm{Cu} \cdots \mathrm{O}$ bonds, 2.5772(12) $\AA$, with oxygen atom belonging to the quinaldinate of the adjacent unit. Fig. 8 shows the ORTEP drawing of a short section of a chain. In this chain, each copper(II) ion is coordinated by four quinaldinates: two are bound in a $\mathrm{N}, \mathrm{O}$-chelating manner and two are bound via one carboxylate oxygen atom. The $\mathrm{N}_{2} \mathrm{O}_{4}$ donors surround the metal ion in the " $4+2$ " bonding pattern. The quinaldinate ligand has employed in coordination all three donor atoms to two metal ions and thereby serves as a bridging ligand. In Harris notation, this binding mode is labelled 2.111. ${ }^{64}$ The quinaldinates substantially deviate from planarity as the angle between the carboxylate moiety and the bicyclic system amounts to $19.22(14)^{\circ}$. Interestingly, a $\pi \cdots \pi$ stacking interaction occurs within the chains between adjacent quinaldinates. Two C-H $\cdots$ O interactions with a length of $2.9530(18) \AA$ may be observed between the quinaldinates of the same $\left[\mathrm{Cu}(\text { quin })_{2}\right]$ unit. 
The novel copper(II) complexes possess two types of amino alcohol ligands: neutral molecules and amino alcoholate anions that form upon the deprotonation of $\mathrm{OH}$ group. Amino alcohols invariably coordinate through their amino group either as monodentate ligands or as bidentate chelating or bidentate bridging ligands. In the latter two cases, the $\mathrm{OH}$ group also participates in coordination. In general, the copper(II)to-amine bonds span a narrow interval, 1.900(8)-2.133(7) A. Conversely, large variations may be observed among the $\mathrm{Cu}-\mathrm{OH}$ bonds: they can be as short as 1.995(3) ^ (10e) or as long as 2.609(3) $\AA$ (10e). Among the known copper(II) amino alcohol complexes, a bidentate chelating coordination prevails, whereas a monodentate one through the amino group occurs only rarely. ${ }^{29}$ To the best of our knowledge, there is no example of a bidentate bridging coordination that was encountered in type complexes. As a rule, the amino alcoholate ligands employ in coordination both donor groups. Compound 9 f revealed a bidentate chelating fashion, whereas dinuclear compounds a tridentate bridging one. A survey of the known copper(II) amino alcoholate complexes shows that the coordination with the alkoxide oxygen acting as a bridge between two or three metal ions is the prevailing one. ${ }^{29}$ The bonding pattern of the amino alcoholate ligands in our compounds is uniform: the copper(II)-to-amine bonds are in the 1.980(2)-2.039(3) Å range and the copper(II)-to-alkoxide bonds are 1.9162(10)-1.974(3) А̊.

\subsection{Intermolecular interactions}

As anticipated, the connectivity in the solid state is governed by hydrogen bonds and $\pi \cdots \pi$ stacking interactions. The supramolecular structures are described only briefly and more details are provided in the ESI. $\dagger$ Type a compounds 1a, 6a and 7a share a similar connectivity in the solid state. In 1a, complex molecules are hydrogen bonded via $\mathrm{OH} \cdots \mathrm{COO}^{-}$and $\mathrm{NH}_{2} \cdots \mathrm{COO}^{-}$interactions into infinite supramolecular chains. The shortest $\mathrm{Cu} \cdot \mathrm{Cu}$ contacts in 1a are 5.9728(2) A. These contacts occur within the same chain. The chains pack in a parallel fashion with no significant $\pi \cdots \pi$ stacking interactions between quinaldinate ligands. ${ }^{66}$ The intermetallic distances in $\mathbf{6 a}$ and $7 \mathbf{a}$ are very similar. In $\mathbf{1 1 a}$, the $\mathrm{OH} \cdots \mathrm{COO}^{-}$hydrogen bonds link molecules into chains with the shortest $\mathrm{Cu} \cdots \mathrm{Cu}$ contacts within the chains exceeding 11 A. Due to the presence of 3-amino-1-propanol molecules in 11a-3a1pOH, its solid-state structure differs. An intricate pattern of hydrogen bonding involving both the complex and solvent molecules of crystallization produces a three-dimensional supramolecular network. Interestingly, as in other type a compounds, there are no $\pi \cdots \pi$ stacking interactions between quinaldinate ligands. The chelates $\mathbf{2 b}, \mathbf{8 b}$ and $\mathbf{1 1 b}$ share the same connectivity pattern: the $\mathrm{OH} \cdots \mathrm{COO}^{-}$and $\mathrm{NH}_{2} \cdots \mathrm{COO}^{-}$synthons link molecules into chains with $\pi \cdots \pi$ stacking occurring between adjacent chains. The structures of $1 \mathrm{D}$-polymers $\mathbf{8 c}$ and $\mathbf{1 1 c}$ are identical: the chains are hydrogen bonded into layers with $\pi \cdots \pi$ stacking occurring between adjacent layers.

The syn disposition of aromatic ligands in $\operatorname{syn}$ - $\left[\mathrm{Cu}_{2} \text { (quin }\right)_{2}-$ $\left.(3 \mathrm{a} 1 \mathrm{pO})_{2}\right]$ (syn-11d) allows intramolecular $\pi \cdots \pi$ stacking. Only weak hydrogen bonds may be observed between dinuclear complex molecules. These bonds link molecules into supramolecular chains. The presence of water molecules of crystallization in $\boldsymbol{s y n - 1 1 d} \cdot \mathbf{2} \mathbf{H}_{2} \mathrm{O}$ entails changes in the connectivity. Hydrogen bonds between water and complex molecules produce infinite layers. A comparison with the complex molecule of $\boldsymbol{s y n - 1 1 d}$ reveals that in $\boldsymbol{s y n - 1 1 d} \cdot 2 \mathrm{H}_{2} \mathrm{O}$ the quinaldinate ligands within the same complex molecule are slightly pulled apart. Furthermore, intermolecular $\pi \cdots \pi$ stacking may be observed. In syn- $\left.\left[\mathrm{Cu}_{2} \text { (quin }\right)_{2}(2 \mathrm{a} 1 \mathrm{pO})_{2}\right]$ (syn-8d), hydrogen bonds between $\mathrm{NH}_{2}$ and $\mathrm{COO}^{-}$entities link complex molecules into double chains. Both types of $\pi \cdots \pi$ stackings may be observed, intra- and intermolecular ones, the latter occurring within the

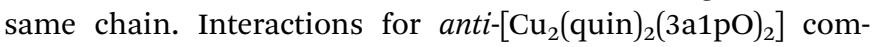
pounds are different since intramolecular $\pi \cdots \pi$ stacking is not possible. In the acetonitrile solvate, anti-11d-2MeCN, the $\mathrm{NH}_{2} \cdots \mathrm{COO}^{-}$synthons link complex molecules into infinite layers. In addition, the complex molecules are held together with intermolecular $\pi \cdots \pi$ stacking within the same layer. Acetonitrile solvent molecules are packed in-between layers. The presence of the solvent, capable of forming hydrogen bonds, changes the connectivity pattern. Both anti-11d-4MeOH and anti-11d-2MeOH·2(3a1pOH) contain such solvents. In the

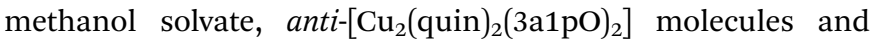
methanol molecules are linked into a three-dimensional supramolecular network in which the layers of complex molecules alternate with the layers of solvent molecules. In anti11d.2MeOH·2(3a1pOH) with two solvents of crystallization, the dinuclear complex molecules are hydrogen bonded into layers. Within the layer, intermolecular $\pi \cdots \pi$ stacking interactions may be observed. The surface of layers is decorated with methanol and 3-amino-1-propanol molecules. Again, the resulting three-dimensional connectivity pattern may be viewed as an alternation of layers of complex molecules and layers of

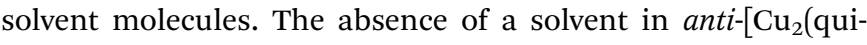
$\left.\mathrm{n})_{2}(2 \mathrm{eaeO})_{2}\right]$ (anti-4d) makes the compound stable and results in a different connectivity pattern: the complex molecules are linked into chains via the $\mathrm{NH}_{2} \cdots \mathrm{COO}^{-}$hydrogen bonds. The chains pack in a parallel fashion with $\pi \cdots \pi$ stacking interactions between adjacent chains.

DFT calculations show the isolated syn isomer of $\left[\mathrm{Cu}_{2} \text { (quin }\right)_{2}-$ $\left.(3 \mathrm{a} 1 \mathrm{pO})_{2}\right]$ to be the most stable by $7.2 \mathrm{kcal} \mathrm{mol}^{-1}(\triangle Z P E)$. This can be almost entirely pinpointed to the intramolecular $\pi \cdots \pi$ stacking as confirmed by the analysis of non-covalent interactions (NCI, Fig. 9). This analysis shows NCIs as isosurfaces in which the electron density $(\rho)$, its reduced gradient and the second largest eigenvalue of Hessian matrix of the electron density $\left(\lambda_{2}\right)$ are close to zero. ${ }^{61}$ On the other hand, the calculations in the absence of empirical dispersion yield both isomers isoenergetic.

Type e compounds share the same connectivity in the solid state. For example, in $\mathbf{5 e}$, the $\left[\mathrm{Cu}(1 \mathrm{a} 2 \mathrm{pOH})_{3}\right]^{2+}$ complex cations and the quinaldinate counterions are hydrogen bonded into supramolecular chains with $\pi \cdots \pi$ stacking interactions between adjacent chains. The solid-state structure of 9 f consists of the $\left[\mathrm{Cu}(2 \mathrm{a} 2 \mathrm{~m} 1 \mathrm{pO})_{2}\right]$ complex molecules, $2 \mathrm{a} 2 \mathrm{~m} 1 \mathrm{pOHH}^{+}$ cations and quin ${ }^{-}$anions that are hydrogen bonded into layers 


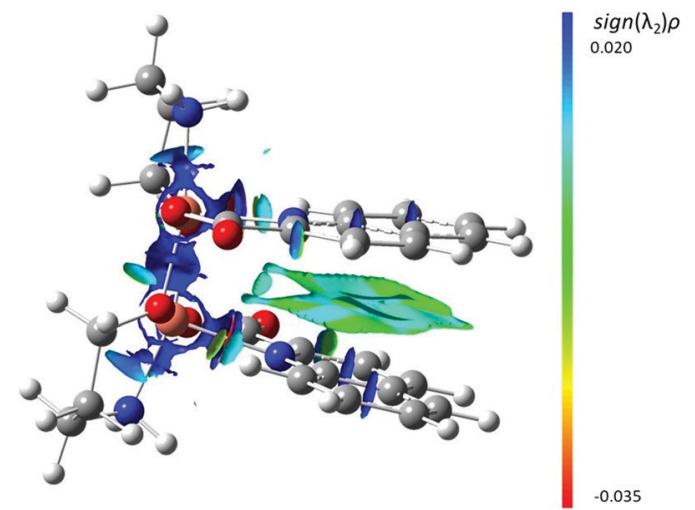

Fig. 9 The $\mathrm{NCl}$ plot showing intramolecular $\pi \cdots \pi$ interactions in syn$\left[\mathrm{Cu}_{2}(\text { quin })_{2}(3 \mathrm{a} 1 \mathrm{pO})_{2}\right]$ with blue and red regions representing repulsive and attractive weak interactions, respectively. Intermediate, weak noncovalent interactions are shown in green.

with $\pi \cdots \pi$ stacking interactions between the layers. In the structure of $\mathbf{g}$, infinite chains pack in a parallel fashion: each chain is surrounded by six others. $\pi \cdots \pi$ stacking interactions occur within the same chain. No classical hydrogen bonds may be observed.

\subsection{Relative stability of the $3 \mathrm{a} 1 \mathrm{pOH}$ monomers}

As the 3-amino-1-propanol ligand produces the richest system in terms of the isolated species, two mononuclear complexes, trans-[Cu(quin $\left.)_{2}(3 \mathrm{a} 1 \mathrm{pOH})_{2}\right] \quad$ (11a) and $\left[\mathrm{Cu}(\text { quin })_{2}(3 \mathrm{a} 1 \mathrm{pOH})\right]$ (11b), were optimized by DFT methods in bulk acetonitrile in order to assess their relative stabilities. The choice of 11a was governed by its reproducible formation under very diverse conditions. On the other hand, the difficulties experienced during the isolation of the two mono amino alcohol compounds, 11b and 11c, implied their nature to be very labile. The calculations indeed show 11a to be the most stable mononuclear species in this system. Furthermore, they indicate that the formal extrusion of one $3 \mathrm{ap} 1 \mathrm{OH}$ ligand from trans$\left[\mathrm{Cu}(\text { quin })_{2}(3 \mathrm{a} 1 \mathrm{pOH})_{2}\right](\mathbf{1 1 a})$ to yield $\left[\mathrm{Cu}(\text { quin })_{2}(3 \mathrm{a} 1 \mathrm{pOH})\right](\mathbf{1 1 b})$ with the remaining amino alcohol bound in a bidentate chelating manner is slightly exergonic (by ca. $1 \mathrm{kcal} \mathrm{mol}^{-1}$; see Table S15 for details, ESI $\dagger$ ). The process can be considered to occur through a five-coordinate species, labelled 11a-L. The latter was derived from a repeating unit of the 1D-polymer 11c.

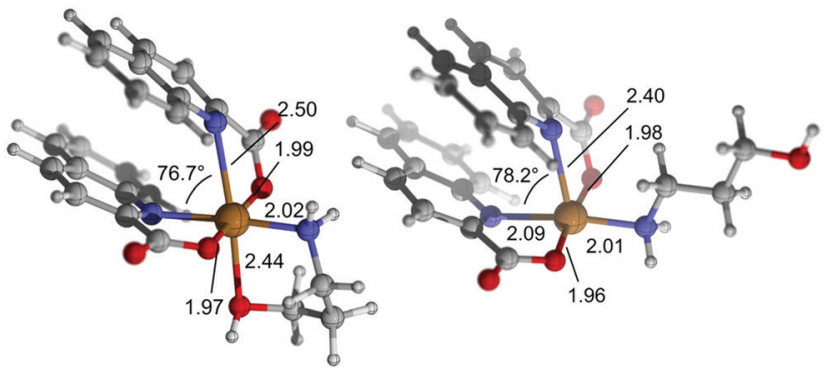

Fig. 10 DFT-optimized geometries of 11b (left) and 11a-L (right).

of one 3a1pOH ligand from 11a. Nevertheless, the energy variations calculated for the interconversion of the above species can be regarded as small enough and as such consistent with their facile interconversion in solution.

As for the greater calculated stability of 11a-L vs. 11b, it must be noted that both species are almost isoenergetic when zero-point energies are compared. However, there is an entropy penalty related to the formation of the chelate $\mathbf{1 1 b}$, which is counterbalanced by the energy released in the formation of a $\mathrm{Cu}-\mathrm{OH}$ bond. This bond is significantly longer (by ca. $0.5 \AA$ ) than the other two $\mathrm{Cu}-\mathrm{O}$ bonds, and the same is true for the $\mathrm{Cu}-\mathrm{N}$ bond trans to it. The elongation of both bonds is attributable to the Jahn-Teller distortion. Accordingly, the Atom in Molecules (AIM) analysis ${ }^{58}$ of the calculated electron density reveals electron densities at the bond critical points of these elongated bonds that are $c a .0 .03$ a.u., compared to the electron densities at the bond critical points for the remaining coordination bonds, which are in the range of 0.06-0.08 a.u. The calculated structures of $\mathbf{1 1 b}$ and $11 \mathbf{a}-\mathbf{L}$ feature marked deviations from their respective ideal octahedral or square pyramid coordination environments (and from the initial X-ray geometries) caused by intramolecular $\pi \cdots \pi$ stacking of quinaldinates (Fig. 10). In the crystal lattice of $\mathbf{1 1 b}$, intermolecular $\pi \cdots \pi$ stacking may be observed instead.

\subsection{Magnetic properties}

The magnetic properties of a series of copper(II) complexes were studied by dc susceptibility measurements between 300 and $1.85 \mathrm{~K}$. The mononuclear compounds, which were measured are $\left[\mathrm{Cu}(\text { quin })_{2}(2 \mathrm{aeOH})_{2}\right](\mathbf{1 a}),\left[\mathrm{Cu}(\text { quin })_{2}(1 \mathrm{a} 2 \mathrm{~m} 2 \mathrm{pOH})_{2}\right]$ (6a), $\left[\mathrm{Cu}(\text { quin })_{2}(1 \mathrm{a} 2 \mathrm{bOH})_{2}\right] \quad$ (7a) $\left[\mathrm{Cu}(\text { quin })_{2}(3 \mathrm{a} 1 \mathrm{pOH})_{2}\right] \quad$ (11a),

$$
\text { trans }-\left[\mathrm{Cu}(\text { quin })_{2}(3 \mathrm{a} 1 \mathrm{pOH})_{2}\right](\mathbf{1 1 a}) \rightarrow\left[\mathrm{Cu}(\text { quin })_{2}(3 \mathrm{a} 1 \mathrm{pOH})\right](\mathbf{1 1 a}-\mathbf{L})+3 \mathrm{a} 1 \mathrm{pOH}
$$

$$
11 \mathrm{a}-\mathrm{L} \rightarrow 11 \mathrm{~b}
$$

The calculations show 11a-L and $3 \mathrm{ap} 1 \mathrm{OH}$ to be almost isoenergetic with 11a. The results on the relative stabilities of 11a, 11a-L and 11b merit further comment since the calculated free energy differences point towards exclusive formation of 11a-L in the solution. The free energy variations were calculated at $1 \mathrm{~atm}$ for all species and intermolecular interactions in solution were not considered. However, the entropy term more than compensates for the energy required for the dissociation
$\left[\mathrm{Cu}(\text { quin })_{2}(2 \mathrm{maeOH})\right] \quad(\mathbf{2 b}), \quad\left[\mathrm{Cu}(1 \mathrm{a} 2 \mathrm{pOH})_{3}\right](\text { quin })_{2} \quad$ (5e), $\left[\mathrm{Cu}(1 \mathrm{a} 2 \mathrm{bOH})_{3}\right](\text { quin })_{2}$ (7e), $\left[\mathrm{Cu}(2 \mathrm{a} 1 \mathrm{pOH})_{3}\right](\text { quin })_{2} \quad(8 \mathbf{e})$ and $\left[\mathrm{Cu}(2 \mathrm{a} 2 \mathrm{~m} 1 \mathrm{pO})_{2}\right](2 \mathrm{a} 2 \mathrm{~m} 1 \mathrm{pOHH})_{2}(\text { quin })_{2}$ (9f). Their room temperature $\chi T$ product values fall within the 0.38 to $0.43 \mathrm{~cm}^{3} \mathrm{~K} \mathrm{~mol}^{-1}$ interval and are consistent with the presence of one $S=1 / 2$ $\mathrm{Cu}(\mathrm{II})$ center with $g$ values ranging from 2.03(5) to 2.15(5). Furthermore, the series of $\mathbf{a}, \mathbf{b}, \mathbf{e}$ and $\mathbf{f}$ compounds displays a very similar temperature dependence of $\chi T$ products (Fig. 11, top part). On lowering the temperature, the $\chi T$ value remains constant at first, whereas at low temperatures, it decreases in a 

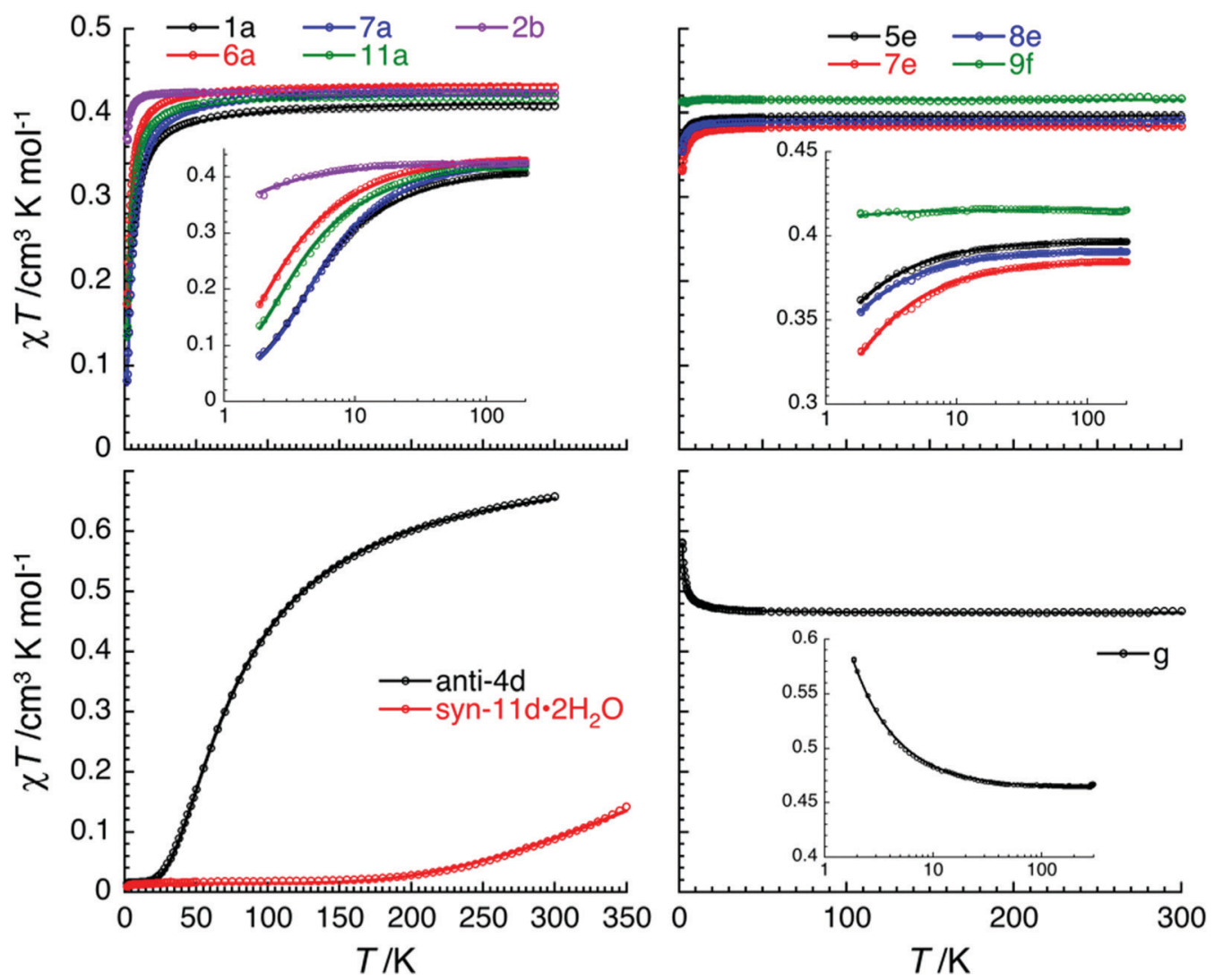

Fig. 11 The temperature dependence of the $\chi T$ product (where $\chi$ is the molar magnetic susceptibility that equals $M / H$ per compound, and $T$ is the temperature), collected at a dc magnetic field of $0.1 \mathrm{~T}$ for $\mathbf{1 a}, \mathbf{6 a}, \mathbf{7 a}, \mathbf{1 1 a}$ and $\mathbf{2 b}$ (top left), $\mathbf{5 e}, \mathbf{7 e}, \mathbf{8 e}$ and $\mathbf{9 f}$ (top right), anti-4d and syn-11d.2 $\mathrm{H}_{2} \mathrm{O}$ (bottom left), and $\mathbf{g}$ (bottom right). Insets: Semi-logarithm $\chi T$ vs. T plots emphasizing the magnetic data at low temperatures. The solid lines are the best fits to the models described in the text.

more or less pronounced manner. Such a behavior is an indication of weak or very weak intermolecular antiferromagnetic (AF) interactions. The solid-state structures of type a compounds are characterized by a hydrogen bonded one-dimensional arrangement with short $\mathrm{Cu}-\mathrm{O} \cdots \mathrm{HN}-\mathrm{Cu}$ contacts (Fig. S4, S5 and Table S5, ESI $\dagger$ ). Since the strongest magnetic interaction was expected to be mediated through these contacts, the magnetic susceptibility was modeled using the regular antiferromagnetic quantum $S=1 / 2$ spin chain model with the following spin Hamiltonian, $\mathcal{H}=-2 J \sum_{i} \vec{S}_{i} \cdot \vec{S}_{i+1}$, and the analytical susceptibility of Bonner-Fisher. ${ }^{67-69}$ As shown in Fig. 11, the magnetic data for 1a, 6a, 7a and 11a are well-reproduced through the whole temperature range with this approach, leading to $J / k_{\mathrm{B}}=$ $-2.6(1) \mathrm{K}$ and $g=2.10(5)$ for $1 \mathrm{a}, J / k_{\mathrm{B}}=-1.4(1) \mathrm{K}$ and $g=2.15(5)$ for $6 \mathrm{a}, J / k_{\mathrm{B}}=-2.8(1) \mathrm{K}$ and $g=2.15(5)$ for $7 \mathrm{a}$, and $J / k_{\mathrm{B}}=$ $-1.75(4) \mathrm{K}$ and $g=2.13(5)$ for 11a.

In $2 \mathbf{b}, 5 \mathbf{e}, 7 \mathbf{e}, \mathbf{8 e}$ and $9 \mathbf{f}$, the mononuclear compounds of types $\mathbf{b}$, e and $\mathbf{f}$, the observed supramolecular contacts and possible magnetic pathways are much weaker and involve more than three atoms between spin carriers. Similarly to type a compounds, a supramolecular chain organization is observed for $2 \mathbf{b}, 5 \mathbf{e}, 7 \mathbf{e}$ and $\mathbf{8 e}$ (Fig. S7 and S16, ESI $\dagger$ ). The shortest $\mathrm{Cu} \cdot \mathrm{Cu}$ contacts in $\mathbf{2 b}$ are $7.0950(2) \AA$ and the ones in type e compounds are in the 6.5514(6)-6.8633(7) ^ range. The magnetic susceptibility for $\mathbf{2 b}, \mathbf{5 e}, 7 \mathbf{e}$ and $\mathbf{8 e}$ was modeled by applying the regular antiferromagnetic quantum $S=1 / 2$ spin chain model ${ }^{67-69}$ which reproduces very well the experimental data (Fig. 11, top part). The corresponding magnetic parameters are $J / k_{\mathrm{B}}=-0.21(5) \mathrm{K}$ and $g=2.13(5)$ for $2 \mathrm{~b}, J / k_{\mathrm{B}}=$ $-0.14(3) \mathrm{K}$ and $g=2.06(5)$ for $5 \mathrm{e}, J / k_{\mathrm{B}}=-0.24(5) \mathrm{K}$ and $g=2.03(5)$ for $7 \mathrm{e}$, and $J / k_{\mathrm{B}}=-0.14(2) \mathrm{K}$ and $g=2.04(5)$ for $8 \mathrm{e}$. The magnetic interactions in these compounds are about 10 times smaller than in type a compounds. In the case of $\mathbf{9 f}$, owing to the co-crystallization of $2 \mathrm{a} 2 \mathrm{~m} 1 \mathrm{pOHH}^{+}$and quin ${ }^{-}$ions, the mononuclear $\left[\mathrm{Cu}(2 \mathrm{a} 2 \mathrm{~m} 1 \mathrm{pO})_{2}\right]$ complex molecules are wellseparated, as shown by the shortest $\mathrm{Cu} \cdot \mathrm{Cu}$ contacts which exceed 8.1133(4) $\AA$. Therefore, it is difficult to imagine any significant magnetic interactions. Magnetic interactions of $-0.01(1) \mathrm{K}$ were thus estimated from the mean field theory (with 4 next-neighbours) and the Weiss constant of $-0.02 \mathrm{~K}$, obtained from the Curie-Weiss fit of the experimental data. It should be underlined that the efficient separation of the $\left[\mathrm{Cu}(2 \mathrm{a} 2 \mathrm{~m} 1 \mathrm{pO})_{2}\right]$ complex molecules in 9 f leads to virtually magnetically isolated $S=1 / 2 \mathrm{Cu}(\mathrm{II})$ spins.

The magnetic properties of the dinuclear $\mathrm{Cu}(\mathrm{II})$ complexes, anti- $\left.\left[\mathrm{Cu}_{2} \text { (quin }\right)_{2}(2 \mathrm{eaeO})_{2}\right]$ (anti-4d) and $s y n-\left[\mathrm{Cu}_{2} \text { (quin) }\right)_{2}(3 \mathrm{a} 1-$ pO $\left.)_{2}\right] \cdot 2 \mathrm{H}_{2} \mathrm{O}\left(\boldsymbol{s y n}-\mathbf{1 1 d} \cdot 2 \mathrm{H}_{2} \mathrm{O}\right)$, were also studied in the $1.85-300 \mathrm{~K}$ 
temperature range (Fig. 11). In both, a pair of $\mathrm{Cu}(\mathrm{II})$ sites is bridged by two alkoxide oxygens. It has been well-documented in the literature ${ }^{70-74}$ that in this type of complexes the nature and magnitude of the magnetic interaction between the $S=1 / 2 \mathrm{Cu}$ spins depend strongly on subtle differences in the geometry of the $\mathrm{Cu}(\mu-\mathrm{OR})_{2} \mathrm{Cu}$ moiety. The $\mathrm{Cu}-(\mu-\mathrm{O})-\mathrm{Cu}$ angle $(\theta)$ was identified as the primary factor: for angles smaller than $95.7^{\circ}$, ferromagnetic couplings are expected, while antiferromagnetic ones are observed for larger angles. ${ }^{70-74}$ Other geometric factors, such as the planarity of the $\mathrm{Cu}_{2}(\mu-\mathrm{O})_{2}$ moiety and the out-of-plane shift of the carbon atom of the bridging alkoxide, known as $\tau$, usually add minor corrections to the main effect of the $\theta$ angle. It was also shown that small values of $\theta$ are associated with larger values of $\tau$. In syn-11d $2 \mathbf{H}_{2} \mathrm{O}$ with $\theta$ being $103.25(8)$ and $103.85(8)^{\circ}$ and $\tau$ being 3.85(17) and 3.99(16) ${ }^{\circ}$, a strong AF interaction is expected, while a weaker one is likely for anti-4d with $\theta=100.09(9)^{\circ}$ and $\tau=38.0(2)^{\circ}$. The room temperature $\chi T$ value, $0.089 \mathrm{~cm}^{3} \mathrm{~K} \mathrm{~mol}^{-1}$ for $\boldsymbol{s y n}-\mathbf{1 1 d} \cdot 2 \mathrm{H}_{2} \mathrm{O}$ and $0.66 \mathrm{~cm}^{3} \mathrm{~K} \mathrm{~mol}^{-1}$ for $\boldsymbol{a n t i - 4 d}$, is lower than the one expected for two magnetically isolated $S=1 / 2$ spins $\left(0.75 \mathrm{~cm}^{3} \mathrm{~K} \mathrm{~mol}^{-1}\right)$, confirming the presence of dominant $\mathrm{AF}$ interactions. On lowering the temperature, the $\chi T$ product decreases. Below $20 \mathrm{~K}$, it vanishes to nearly zero for both compounds, indicating that strong antiferromagnetic interactions induce a singlet ground state, $S_{\mathrm{T}}=0$. To evaluate the intradimer magnetic interaction, the magnetic data were fitted to a simple $S=1 / 2$ spin dimer model with the following Hamiltonian, $\mathcal{H}=-2 J\left(\vec{S}_{1} \cdot \vec{S}_{2}\right)$, and the analytical susceptibility expression of Bleaney and Bowers. ${ }^{75}$ An excellent agreement between the experimental data and the model is observed with $J / k_{\mathrm{B}}=$ $-550(10) \mathrm{K}$ and $g=2.1$ (fixed) for $\boldsymbol{s y n}-\mathbf{1 1 d} \cdot 2 \mathbf{H}_{2} \mathbf{O}$, and $J / k_{\mathrm{B}}=$ $-69.5(5) \mathrm{K}$ and $g=2.01(5)$ for anti-4d. It is worth mentioning that as usually done for systems with a singlet ground state, Curie impurities have been included in this model allowing the estimation of their presence at 3.7 and $3.8 \%$ of a $S=1 / 2$ spin.

Singlet and triplet states of $s y n$ - $\left[\mathrm{Cu}_{2}(\text { quin })_{2}(3 \mathrm{a} 1 \mathrm{pO})_{2}\right]$ were calculated by optimizing the X-ray structure without restrictions in bulk acetonitrile. According to $-2 J=E(S=1)-E\left(S_{\mathrm{T}}=0\right),{ }^{72}$ the sign and the magnitude of the magnetic coupling constant can be estimated as $-163.1 \mathrm{~K}$. The calculations predict a singlet ground state also for an anti isomer, although no direct bonding was located between the copper(II) ions by AIM analysis in either case (Fig. 12). ${ }^{58}$ The calculated value of the magnetic coupling constant for isolated $s y n$ - $\left[\mathrm{Cu}_{2}(\text { quin })_{2}(3 \mathrm{a} 1 \mathrm{pO})_{2}\right]$ differs from the experimental one for bulk syn-11d $2 \mathbf{H}_{2} \mathbf{O}$. For the optimized syn$\left.\left[\mathrm{Cu}_{2} \text { (quin) }\right)_{2}(3 \mathrm{a} 1 \mathrm{pO})_{2}\right], \theta$ amounts to $97.8^{\circ}$ and $\tau$ to $43^{\circ}$. In line with the previous discussion, a smaller value of $\theta$ is, as expected, associated with a larger $\tau$ and a weaker AF interaction.

The magnetic susceptibility of $\left[\mathrm{Cu}(\text { quin })_{2}\right]_{n}$ (g) was also studied. This compound features an infinite chain structure with a $4.8617(2) \AA$ distance between adjacent copper(II) ions, bridged by two anti(eq)-syn(ax)-carboxylate groups. On lowering the temperature down to $60 \mathrm{~K}$, the $\chi T$ product is quasi-constant with the $0.46 \mathrm{~cm}^{3} \mathrm{~K} \mathrm{~mol}^{-1}$ value being in good agreement with the presence of a $S=1 / 2 \mathrm{spin}$. At lower temperatures, $\chi T$ slightly increases (up to $0.58 \mathrm{~cm}^{3} \mathrm{~K} \mathrm{~mol}^{-1}$ at $1.85 \mathrm{~K}$ and $0.1 \mathrm{~T}$ )

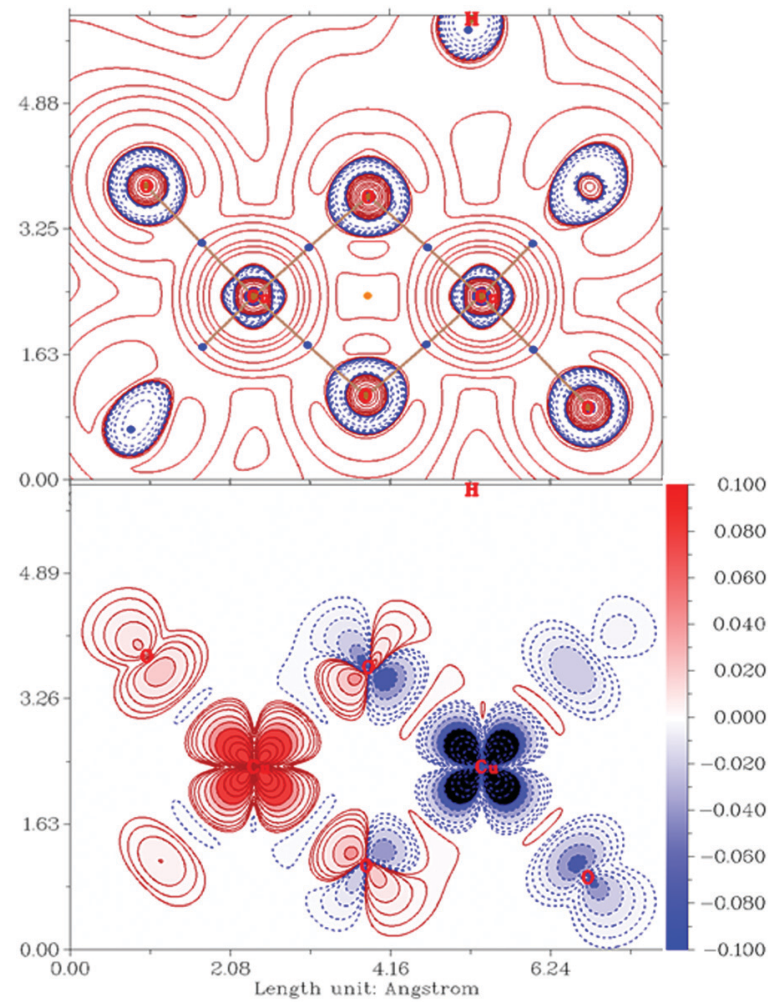

Fig. 12 Bond critical points (bcps) and corresponding bond paths of the calculated electron density of the singlet syn- $\left[\mathrm{Cu}_{2}(\text { quin })_{2}(3 \mathrm{a} 1 \mathrm{pO})_{2}\right]$ in the $\mathrm{Cu}_{2}(\mu-\mathrm{O})_{2}$ plane. The contour map of the Laplacian of the electron density $\left(\nabla^{2} \rho\right)$ is also plotted (above); the electron spin-density plot for the singlet ground state of the same species (below). Solid lines and red tones correspond to positive values whereas dashed lines and blue tones are for negative values.

suggesting dominant, but very weak, ferromagnetic interactions between spin carriers. A Heisenberg chain model of ferromagnetically coupled $S=1 / 2$ spins with the following spin Hamiltonian, $\mathcal{H}=-2 J \sum_{i} \vec{S}_{i} \cdot \vec{S}_{i+1}$, was employed to fit the experimental data. Using the analytical expression of susceptibility proposed by Baker et al., ${ }^{76}$ the experimental data are remarkably well reproduced down to $1.85 \mathrm{~K}$ with $J / k_{\mathrm{B}}=+0.04(1) \mathrm{K}$ and $g=2.22(5)$. As expected, the double carboxylate bridges mediate weak ferromagnetic interactions between $S=1 / 2 \mathrm{Cu}$ (II) spins. $^{77}$

\subsection{Infrared spectroscopy}

The presence of quinaldinate and amino alcohol ligands is clearly evident from infrared spectra. Owing to the overlap of some of their characteristic absorption bands and the engagement of $\mathrm{NH}_{2}$ and $\mathrm{OH}$ groups in hydrogen bonds, not all relevant features of the two ligand types can be unambiguously assigned. It may be noted that compounds with the same solidstate structure have very similar infrared spectra. The identification of the quinaldinate absorptions was facilitated by the spectrum of $\left[\mathrm{Cu}(\text { quin })_{2}\right]_{n}$ (g), a homoleptic complex. Frequencies of the $\nu_{\mathrm{as}}\left(\mathrm{COO}^{-}\right)$and $\nu_{\mathrm{s}}\left(\mathrm{COO}^{-}\right)$vibrational modes, listed in Table S18 (ESI $\dagger$ ), are of particular importance as they are 
sensitive to the carboxylate binding mode. ${ }^{78}$ The difference in their positions is known as the $\Delta$ value. Magnitudes of $\Delta$ for all our compounds with monodentate carboxylates are in line with the expectations. ${ }^{78}$ However, the $\Delta$ criterion fails for $\left[\mathrm{Cu}(\text { quin })_{2}\right]_{n}$ (g) as the frequency difference amounts to $272 \mathrm{~cm}^{-1}$. Its magnitude is larger than expected for a bidentate bridging carboxylate coordination. $^{78}$ In the case of $\left[\mathrm{Cu}(2 \mathrm{a} 2 \mathrm{~m} 1 \mathrm{pO})_{2}\right](2 \mathrm{a} 2 \mathrm{~m} 1 \mathrm{pOHH})_{2}{ }^{-}$ (quin) $)_{2}$ (9f), only non-coordinated quinaldinate ions are present. Their role of counter anions is evident from the spectrum: the $\nu_{\text {as }}\left(\mathrm{COO}^{-}\right)$vibration is associated with the band at $1553 \mathrm{~cm}^{-1}$ and that of the $\nu_{\mathrm{s}}\left(\mathrm{COO}^{-}\right)$vibration with the band at $1372 \mathrm{~cm}^{-1}$. As a result, the value of $\Delta$ amounts to $181 \mathrm{~cm}^{-1}$. The ionic type $\mathrm{e}$ compounds exhibit carboxylate bands at almost the same positions as 9f. It should be noted that a set of four peaks with medium intensity, i.e., at 1567, 1510, 1463 and $1436 \mathrm{~cm}^{-1}$ for $\mathbf{g}$, invariably appears in the spectra of all quinaldinate complexes at approximately the same frequencies.

A close survey of the infrared data of our copper(II) compounds shows that no information about the binding mode of the amino alcohol ligand can be extracted from their spectra. The $\nu(\mathrm{N}-\mathrm{H})$ and $\nu(\mathrm{O}-\mathrm{H})$ vibrations can be told apart only in rare cases. Due to extensive participation of $\mathrm{NH}_{2}$ and $\mathrm{OH}$ groups in hydrogen bonding, $\mathrm{N}-\mathrm{H}$ and $\mathrm{O}-\mathrm{H}$ stretches often appear as broad bands. ${ }^{79}$ The expected region for the $\mathrm{NH}_{2}$ deformation band in primary amines, $1650-1590 \mathrm{~cm}^{-1},{ }^{79}$ overlaps with the most intense quinaldinate signature band, the $\nu_{\text {as }}\left(\mathrm{COO}^{-}\right)$band. Therefore, these bands could not be unambiguously assigned. The presence of alcohol in the compound may be seen in another spectral region: the region of $\mathrm{C}-\mathrm{O}$ stretching absorptions. The $\nu(\mathrm{C}-\mathrm{O})$ absorptions are seen as strong bands with their frequencies sensitive to the nature of the alcohol. ${ }^{79}$ The alcohols in the bonded state are reported to absorb very broadly and diffusely near $650 \mathrm{~cm}^{-1}$ owing to the out-of-plane $\mathrm{C}-\mathrm{OH}$ deformation. ${ }^{79}$ Our compounds with amino alcohols also reveal a band of medium intensity in this region (Table S19, ESI $\dagger$ ).

The spectra of type a complexes are the least uniform: the number and the frequencies of the bands in the $\nu(\mathrm{N}-\mathrm{H}) / \nu(\mathrm{O}-\mathrm{H})$ region differ for all. The $\nu(\mathrm{C}-\mathrm{O})$ spectral region reveals intense bands: 1a with a primary alcohol $2 \mathrm{aeOH}$ features a band at $1034 \mathrm{~cm}^{-1}$, 7a with a secondary alcohol 1a2bOH absorbs at $1137-961 \mathrm{~cm}^{-1}$ and $6 \mathrm{a}$ with a tertiary alcohol $1 \mathrm{a} 2 \mathrm{~m} 2 \mathrm{pOH}$ displays several bands in the $1178-958 \mathrm{~cm}^{-1}$ region. One of the common spectral features for $1 \mathbf{1 a}, \mathbf{6 a}, 7 \mathbf{a}$ and $11 \mathbf{a}$ is four absorptions in the region of $\nu_{\text {as }}\left(\mathrm{COO}^{-}\right)$and $\mathrm{N}-\mathrm{H}$ bending vibrational mode, as exemplified by 1604, 1587, 1562 and $1550 \mathrm{~cm}^{-1}$ bands for 1a. The high-frequency band at $c a$. $1604 \mathrm{~cm}^{-1}$ was tentatively assigned to the $\nu_{\text {as }}\left(\mathrm{COO}^{-}\right)$vibration and the rest to the $\mathrm{N}-\mathrm{H}$ bending vibrations. The trans$\left[\mathrm{Cu}(\text { quin })_{2}\right]$ structural fragment leaves its fingerprint in the shape and frequencies of four bands in the out-of-plane $\mathrm{C}-\mathrm{H}$ region. It is to be noted that the crystallization solvent molecules and a different solid-state structure of 11a.3a1pOH make its infrared spectrum markedly different from that of its unsolvated counterpart 11a.

The infrared spectra of type $\mathbf{b}$ compounds are very much alike. For the $\mathbf{8 b}$ and $\mathbf{1 1 b}$ pair, there are four bands in the
$\nu(\mathrm{N}-\mathrm{H}) / \nu(\mathrm{O}-\mathrm{H})$ region. Owing to the secondary nature of the amino alcohol in $\mathbf{2 b}$, the number of peaks in this region is smaller. The spectra of all three reveal two prominent absorptions in the $\nu_{\text {as }}\left(\mathrm{COO}^{-}\right) / \mathrm{N}-\mathrm{H}$ bending region: for example, the spectrum of $11 \mathrm{~b}$ reveals an intense band at $1641 \mathrm{~cm}^{-1}$ and a band at $1594 \mathrm{~cm}^{-1}$. The same absorption pattern may be observed for the spectra of $\mathbf{8 c}$ and 11c. Again, their similarity can be traced to the similarity of their solid-state structures.

The spectra of the dinuclear complexes should be free of $\nu(\mathrm{O}-\mathrm{H})$ absorptions in keeping with the formulation as an amino alcoholate rather than an alcohol complex. This expectation is realized for compounds that do not contain solvent molecules of crystallization, i.e., methanol, 3-amino-1-propanol

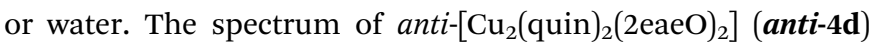
differs from the spectra of the $3 \mathrm{a} 1 \mathrm{pO}^{-}$anti dimers, anti11d.4MeOH, anti-11d·2MeCN and anti-11d·2MeOH·2(3a1pOH). In the spectrum of anti-4d, the $\nu(\mathrm{N}-\mathrm{H})$ absorption occurs as a single sharp band at $3183 \mathrm{~cm}^{-1}$. Broad bands, belonging to solvent molecules of 3-amino-1-propanol and/or methanol for anti-11d·2MeOH·2(3a1pOH) and anti-11d·4MeOH, mask the $\nu(\mathrm{N}-\mathrm{H})$ bands of the coordinated amino alcoholate. The presence of solvent molecules is apparent also from the characteristic region of the $\mathrm{C}-\mathrm{O}$ stretching vibrations: for anti-11d-4MeOH, a very intense band may be observed at $1022 \mathrm{~cm}^{-1}$, whereas for anti-11d·2MeOH·2(3a1pOH) a band occurs at $1051 \mathrm{~cm}^{-1}$. Their intensities diminish with time, in line with the rapid loss of solvent molecules. The presence of the acetonitrile solvent molecules in anti-11d-2MeCN is ascertained by the sharp band at $2249 \mathrm{~cm}^{-1} \cdot{ }^{79}$ The spectra of $\boldsymbol{s y n - 8 d ,} \boldsymbol{s y n - 9 d}$ and $\boldsymbol{s y n - 1 0 d}$ are very much alike. It is to be noted that unambiguous identification by means of X-ray crystallography was accomplished only for syn-8d. Based on the similarity of their spectra, it can be claimed with a high probability that their solid-state structures are very similar. All three reveal two sharp bands that can be attributed to $\nu(\mathrm{N}-\mathrm{H})$ absorptions. For syn-8d, the peaks occur at 3251 and $3145 \mathrm{~cm}^{-1}$. $\boldsymbol{s y n}-11 \mathrm{~d} \cdot 2 \mathbf{H}_{2} \mathrm{O}$ also features two bands at almost the same frequencies. The water solvate has an additional broad band at $3469 \mathrm{~cm}^{-1}$ whose origin lies in the $\nu(\mathrm{O}-\mathrm{H})$ vibrations of solvent water molecules.

The spectral features of type e ionic compounds with chelating amino alcohol ligands are very similar. They display two bands in the $\nu(\mathrm{N}-\mathrm{H}) / \nu(\mathrm{O}-\mathrm{H})$ region, at 3236 and $3122 \mathrm{~cm}^{-1}(5 \mathbf{e})$. Surprisingly, in spite of the same binding mode employed by the amino alcohol ligands, the spectra are markedly different from the spectra of type $\mathbf{b}$ complexes. The spectrum of $\left[\mathrm{Cu}(2 \mathrm{a} 2 \mathrm{~m} 1 \mathrm{pO})_{2}\right](2 \mathrm{a} 2 \mathrm{~m} 1 \mathrm{pOHH})_{2}$ (quin $)_{2}$ (9f), a compound which contains both the amino alcoholate ligand and the cationic form of the parent amino alcohol, reveals the $\nu(\mathrm{N}-\mathrm{H})$ vibration of the $\mathrm{NH}_{2}$ group as a sharp absorption of medium intensity at $3315 \mathrm{~cm}^{-1}$. A broad absorption that spans the $3200-2500 \mathrm{~cm}^{-1}$ region is attributed to the $\nu(\mathrm{N}-\mathrm{H})$ and $\nu(\mathrm{O}-\mathrm{H})$ absorptions of the hydrogen bonded $\mathrm{NH}_{3}{ }^{+}$and $\mathrm{OH}$ entities. The region of the $\mathrm{N}-\mathrm{H}$ bending vibrations reveals three bands, at 1644, 1602 and $1582 \mathrm{~cm}^{-1}$. The $\nu(\mathrm{C}-\mathrm{O})$ vibrations may be seen as strong bands at 1055 and $1010 \mathrm{~cm}^{-1}$. 


\section{Conclusions}

The $\mathrm{Cu}(\mathrm{II})$-quinaldinate-amino alcohol reaction systems display great structural diversity in their products which may be classified into seven distinctly different structural types. The series encompasses mononuclear, dinuclear and 1D-polymeric species with either intact amino alcohols or amino alcoholate ions serving as ligands. No correlation between the nature of the product and the applied reaction conditions could be discerned. To the best of our knowledge, copper(II) complexes with three out of eleven amino alcohols, 2-methylaminoethanol, 1-amino-2-methyl-2-propanol and 1-amino-2-butanol, have not been prepared prior to this study. The richest system in terms of products was that of 3-amino-1-propanol: it has yielded nine compounds. Two mononuclear 3-amino-1propanol complexes, trans- $\left[\mathrm{Cu}(\text { quin })_{2}(3 \mathrm{a} 1 \mathrm{pOH})_{2}\right]$ and $\left[\mathrm{Cu}(\text { quin })_{2}-\right.$ (3a1pOH)] with a chelating amino alcohol, were subjected to DFT calculations which show their facile interconversion in the solution to proceed via an intermediate five-coordinate species. The dinuclear amino alcoholate complex, $\left[\mathrm{Cu}_{2}(\text { quin })_{2}(3 \mathrm{a} 1 \mathrm{pO})_{2}\right]$, displayed syn- and anti-isomerism. DFT calculations on the isolated isomers revealed the syn isomer as the most stable one with its stability originating from an intramolecular $\pi \cdots \pi$ stacking interaction between quinaldinates. Furthermore, calculations show that both isomers display antiferromagnetic interactions between $S=1 / 2 \mathrm{Cu}(\mathrm{II})$ spins. These results qualitatively agree with the experimental value for syn- $\left.\left[\mathrm{Cu}_{2} \text { (quin }\right)_{2}(3 \mathrm{a} 1 \mathrm{pO})_{2}\right] \cdot 2 \mathrm{H}_{2} \mathrm{O}$. In line with previous studies, the nature and strength of the magnetic exchange depends upon the geometry of the $\mathrm{Cu}(\mu-\mathrm{O})_{2} \mathrm{Cu}$ core. The mononuclear complexes trans- $\left[\mathrm{Cu}(\text { quin })_{2} \mathrm{~L}_{2}\right]$ (where $\mathrm{L}$ denotes amino alcohol) and $\left[\mathrm{Cu}(\text { quin })_{2} \mathrm{~L}\right]$ with a bidentate chelating amino alcohol display very weak to weak antiferromagnetic coupling through supramolecular contacts in their solid-state structures. By analogy, $\left[\mathrm{Cu}(2 \mathrm{a} 2 \mathrm{~m} 1 \mathrm{pO})_{2}\right](2 \mathrm{a} 2 \mathrm{~m} 1 \mathrm{pOHH})_{2}(\text { quin })_{2}$ with an efficient separation of the $S=1 / 2 \mathrm{Cu}$ (II) spins by the counter ions does not display significant magnetic interactions. $\left.[\mathrm{Cu} \text { (quin) })_{2}\right]_{n}$, the homoleptic quinaldinate complex with an infinite chain structure, is the only one in the series that features weak but nevertheless observable, ferromagnetic behavior.

\section{Author contributions}

Conceptualization: B. M. and N. P.; investigation and formal analysis: all authors; writing - review \& editing: B. M., N. P., R. C. and J. L. S.

\section{Funding}

Research was funded by the Slovenian Research Agency (Junior Researcher Grant for N. P. and the Program Grant P1-0134), MICINN (grant PID2019-110856GA-I00), the CNRS, the University of Bordeaux, the Région Nouvelle Aquitaine and Quantum Matter Bordeaux.

\section{Conflicts of interest}

There are no conflicts to declare.

\section{Acknowledgements}

M. M. A. thanks the MICINN for a research fellowship. The Supercomputing Centre of Galicia (CESGA) and the Centro de Servicios de Informática y Redes de Comunicaciones (CSIRC), Universidad de Granada are acknowledged for providing the computing time.

\section{References}

1 M. O'Keeffe, M. A. Peskov, S. J. Ramsden and O. M. Yaghi, Acc. Chem. Res., 2008, 41, 1782-1789.

2 M. O'Keeffe and O. M. Yaghi, Chem. Rev., 2012, 112, 675-702.

3 E. V. Alexandrov, V. A. Blatov, A. V. Kochetkov and D. M. Proserpio, CrystEngComm, 2011, 13, 3947-3958.

4 G. R. Desiraju, J. J. Vittal and A. Ramanan, Crystal Engineering: A Textbook, World Scientific Publishing Co., Singapore, 2011.

5 G. R. Desiraju, Angew. Chem., Int. Ed., 2007, 46, 8342-8356.

6 N. N. Greenwood and A. Earnshaw, Chemistry of the Elements, Butterworth-Heinemann, Oxford, UK, 2nd edn, 1997.

7 K. S. Egorova and V. P. Ananikov, Organometallics, 2017, 36, 4071-4090.

8 J. J. R. Fraústo da Silva and R. J. P. Williams, The biological chemistry of the elements: The inorganic chemistry of life, Oxford University Press, Oxford, UK, 2nd edn, 2001.

9 R. A. Festa and D. J. Thiele, Curr. Biol., 2011, 21, R877-R883.

10 V. Tudor, T. Mocanu, F. Tuna, A. M. Madalan, C. Maxim, S. Shova and M. Andruh, J. Mol. Struct., 2013, 1046, 164-170.

11 J. W. Sharples and D. Collison, Coord. Chem. Rev., 2014, 260, 1-20.

12 P. Seppälä, E. Colacio, A. J. Mota and R. Sillanpää, Dalton Trans., 2012, 41, 2648-2658.

13 S. S. P. Dias, V. André, J. Kłak, M. T. Duarte and A. M. Kirillov, Cryst. Growth Des., 2014, 14, 3398-3407.

14 P. Seppälä, E. Colacio, A. J. Mota and R. Sillanpää, Inorg. Chim. Acta, 2010, 363, 755-762.

15 R. Sillanpää, T. Lindgren and L. Hiltunen, Inorg. Chim. Acta, 1987, 131, 85-88.

16 L. J. Farrugia, D. S. Middlemiss, R. Sillanpää and P. Seppälä, J. Phys. Chem. A, 2008, 112, 9050-9067.

17 G. Marin, V. Kravtsov, Y. A. Simonov, V. Tudor, J. Lipkowski and M. Andruh, J. Mol. Struct., 2006, 796, 123-128.

18 C. Paraschiv, M. Andruh, S. Ferlay, M. W. Hosseini, N. Kyritsakas, J.-M. Planeix and N. Stanica, Dalton Trans., 2005, 1195-1202.

19 G. Marin, M. Andruh, A. M. Madalan, A. J. Blake, C. Wilson, N. R. Champness and M. Schröder, Cryst. Growth Des., 2008, 8, 964-975. 
20 F. Sama, A. K. Dhara, M. N. Akhtar, Y.-C. Chen, M.-L. Tong, I. A. Ansari, M. Raizada, M. Ahmad, M. Shahid and Z. A. Siddiqi, Dalton Trans., 2017, 46, 9801-9823.

21 V. T. Yilmaz, Y. Topcu, F. Yilmaz and C. Thoene, Polyhedron, 2001, 20, 3209-3217.

22 J. Madarász, P. Bombicz, M. Czugler and G. Pokol, Polyhedron, 2000, 19, 457-463.

23 G. Marin, V. Tudor, V. C. Kravtsov, M. Schmidtmann, Y. A. Simonov, A. Müller and M. Andruh, Cryst. Growth Des., 2005, 5, 279-282.

24 G. Xu, X. He, J. Lv, Z. Zhou, Z. Du and Y. Xie, Cryst. Growth Des., 2012, 12, 3619-3630.

25 T. A. Fernandes, M. V. Kirillova, V. André and A. M. Kirillov, Dalton Trans., 2018, 47, 16674-16683.

26 I. Mantasha, M. Shahid, M. Ahmad, Rahisuddin, R. Arif, S. Tasneem, F. Sama and I. A. Ansari, New J. Chem., 2019, 43, 622-633.

27 A. M. Kirillov, Y. Y. Karabach, M. Haukka, M. F. C. Guedes da Silva, J. Sanchiz, M. N. Kopylovich and A. J. L. Pombeiro, Inorg. Chem., 2008, 47, 162-175.

28 I. A. Ansari, F. Sama, M. Raizada, M. Shahid, M. Ahmad and Z. A. Siddiqi, New J. Chem., 2016, 40, 9840-9852.

29 C. R. Groom, I. J. Bruno, M. P. Lightfoot and S. C. Ward, Acta Crystallogr., Sect. B: Struct. Sci., Cryst. Eng. Mater., 2016, 72, 171-179.

30 P. Seppälä, R. Sillanpää and A. Lehtonen, Coord. Chem. Rev., 2017, 347, 98-114.

31 M. Andruh, Pure Appl. Chem., 2005, 77, 1685-1706.

32 M. Andruh, Chem. Commun., 2007, 2565-2577.

33 A. M. Kirillov, M. V. Kirillova and A. J. L. Pombeiro, Coord. Chem. Rev., 2012, 256, 2741-2759.

34 A. M. Kirillov, M. N. Kopylovich, M. V. Kirillova, E. Y. Karabach, M. Haukka, M. F. C. Guedes da Silva and A. J. L. Pombeiro, Adv. Synth. Catal., 2006, 348, 159-174.

35 A. M. Kirillov, M. V. Kirillova, L. S. Shul'pina, P. J. Figiel, K. R. Gruenwald, M. F. C. Guedes da Silva, M. Haukka, A. J. L. Pombeiro and G. B. Shul'pin, J. Mol. Catal. A: Chem., 2011, 350, 26-34.

36 A. M. Kirillov, M. N. Kopylovich, M. V. Kirillova, M. Haukka, M. F. C. Guedes da Silva and A. J. L. Pombeiro, Angew. Chem., Int. Ed., 2005, 44, 4345-4349.

37 N. Podjed, P. Stare, R. Cerc Korošec, M. M. Alcaide, J. LópezSerrano and B. Modec, New J. Chem., 2020, 44, 387-400.

38 D. B. G. Williams and M. Lawton, J. Org. Chem., 2010, 75, 8351-8354.

39 B. Modec, N. Podjed and N. Lah, Molecules, 2020, 25, 1573. 40 Agilent, CrysAlis PRO, Agilent Technologies Ltd, Yarnton, Oxfordshire, England, 2014.

41 O. V. Dolomanov, L. J. Bourhis, R. J. Gildea, J. A. K. Howard and H. Puschmann, J. Appl. Crystallogr., 2009, 42, 339-341.

42 G. M. Sheldrick, Acta Crystallogr., Sect. A: Found. Adv., 2015, 71, 3-8.

43 G. M. Sheldrick, Acta Crystallogr., Sect. C: Struct. Chem., 2015, 71, 3-8.

44 A. L. Spek, Acta Crystallogr., Sect. D: Biol. Crystallogr., 2009, 65, 148-155.
45 L. J. Farrugia, J. Appl. Crystallogr., 2012, 45, 849-854.

46 C. F. Macrae, I. J. Bruno, J. A. Chisholm, P. R. Edgington, P. McCabe, E. Pidcock, L. Rodriguez-Monge, R. Taylor, J. van de Streek and P. A. Wood, J. Appl. Crystallogr., 2008, 41, 466-470.

47 M. J. Frisch, G. W. Trucks, H. B. Schlegel, G. E. Scuseria, M. A. Robb, J. R. Cheeseman, G. Scalmani, V. Barone, B. Mennucci, G. A. Petersson, H. Nakatsuji, M. Caricato, X. Li, H. P. Hratchian, A. F. Izmaylov, J. Bloino, G. Zheng, J. L. Sonnenberg, M. Hada, M. Ehara, K. Toyota, R. Fukuda, J. Hasegawa, M. Ishida, T. Nakajima, Y. Honda, O. Kitao, H. Nakai, T. Vreven, J. A. Montgomery Jr., J. E. Peralta, F. Ogliaro, M. Bearpark, J. J. Heyd, E. Brothers, K. N. Kudin, V. N. Staroverov, T. Keith, R. Kobayashi, J. Normand, K. Raghavachari, A. Rendell, J. C. Burant, S. S. Iyengar, J. Tomasi, M. Cossi, N. Rega, J. M. Millam, M. Klene, J. E. Knox, J. B. Cross, V. Bakken, C. Adamo, J. Jaramillo, R. Gomperts, R. E. Stratmann, O. Yazyev, A. J. Austin, R. Cammi, C. Pomelli, J. W. Ochterski, R. L. Martin, K. Morokuma, V. G. Zakrzewski, G. A. Voth, P. Salvador, J. J. Dannenberg, S. Dapprich, A. D. Daniels, Ö. Farkas, J. B. Foresman, J. V. Ortiz, J. Cioslowski and D. J. Fox, Gaussian 09 (Revisions B.01 and E.01), Gaussian, Inc., Wallingford, CT, 2013.

48 G. A. Petersson, A. Bennett, T. G. Tensfeldt, M. A. Al-Laham, W. A. Shirley and J. Mantzaris, J. Chem. Phys., 1988, 89, 2193-2218.

49 G. A. Petersson and M. A. Al-Laham, J. Chem. Phys., 1991, 94, 6081-6090.

50 D. Andrae, U. Häußermann, M. Dolg, H. Stoll and H. Preuß, Theor. Chim. Acta, 1990, 77, 123-141.

51 A. D. Becke, J. Chem. Phys., 1993, 98, 5648-5652.

52 J. P. Perdew, J. A. Chevary, S. H. Vosko, K. A. Jackson, M. R. Pederson, D. J. Singh and C. Fiolhais, Phys. Rev. B: Condens. Matter Mater. Phys., 1992, 46, 6671-6687.

53 J. P. Perdew, Phys. B, 1991, 172, 1-6.

54 S. Grimme, S. Ehrlich and L. Goerigk, J. Comput. Chem., 2011, 32, 1456-1465.

55 A. V. Marenich, C. J. Cramer and D. G. Truhlar, J. Phys. Chem. B, 2009, 113, 6378-6396.

56 R. Seeger and J. A. Pople, J. Chem. Phys., 1977, 66, 3045-3050.

57 R. Bauernschmitt and R. Ahlrichs, J. Chem. Phys., 1996, 104, 9047-9052.

58 R. F. W. Bader, Atom in Molecules: A Quantum Theory, Clarendon Press, Oxford, UK, 1994.

59 T. Lu and F. Chen, J. Comput. Chem., 2012, 33, 580-592.

60 Multiwfn 3.6, http://sobereva.com/multiwfn/.

61 E. R. Johnson, S. Keinan, P. Mori-Sánchez, J. ContrerasGarcía, A. J. Cohen and W. Yang, J. Am. Chem. Soc., 2010, 132, 6498-6506.

62 C. Y. Legault, CYLview20, Université de Sherbrooke, 2020, http://www.cylview.org.

63 C. E. Housecroft and A. G. Sharpe, Inorganic chemistry, Pearson, Harlow, England, 5th edn, 2018.

64 R. A. Coxall, S. G. Harris, D. K. Henderson, S. Parsons, P. A. Tasker and R. E. P. Winpenny, J. Chem. Soc., Dalton Trans., 2000, 2349-2356. 
65 A. W. Addison, T. N. Rao, J. Reedijk, J. van Rijn and G. C. Verschoor, J. Chem. Soc., Dalton Trans., 1984, 1349-1356.

66 C. Janiak, J. Chem. Soc., Dalton Trans., 2000, 3885-3896.

67 J. C. Bonner and M. E. Fisher, Phys. Rev., 1964, 135, A640-A658.

68 W. E. Estes, D. P. Gavel, W. E. Hatfield and D. J. Hodgson, Inorg. Chem., 1978, 17, 1415-1421.

69 J. W. Hall, W. E. Marsh, R. R. Weller and W. E. Hatfield, Inorg. Chem., 1981, 20, 1033-1037.

70 O. Kahn, Molecular magnetism, Wiley-VCH, New York, 1993.

71 V. H. Crawford, H. W. Richardson, J. R. Wasson, D. J. Hodgson and W. E. Hatfield, Inorg. Chem., 1976, 15, 2107-2110.

72 E. Ruiz, P. Alemany, S. Alvarez and J. Cano, J. Am. Chem. Soc., 1997, 119, 1297-1303.
73 E. Ruiz, P. Alemany, S. Alvarez and J. Cano, Inorg. Chem., 1997, 36, 3683-3688.

74 L. Merz and W. Haase, J. Chem. Soc., Dalton Trans., 1980, 875-879.

75 B. Bleaney and K. D. Bowers, Proc. R. Soc. London, Ser. A, 1952, 214, 451-465.

76 G. A. Baker, G. S. Rushbrooke and H. E. Gilbert, Phys. Rev., 1964, 135, A1272-A1277.

77 M. Murugesu, R. Clérac, B. Pilawa, A. Mandel, C. E. Anson and A. K. Powell, Inorg. Chim. Acta, 2002, 337, 328-336.

78 G. B. Deacon and R. J. Phillips, Coord. Chem. Rev., 1980, 33, 227-250.

79 N. B. Colthup, L. H. Daly and S. E. Wiberley, Introduction to Infrared and Raman Spectroscopy, Academic Press, San Diego, CA, 3rd edn, 1990. 